\title{
Negative Reinforcement by Timeout from Avoidance: The Roles of Discriminative Cues, Shock-Frequency Reduction, and Response- Effort Reduction
}

Anne M. Foreman

West Virginia University

Follow this and additional works at: https://researchrepository.wvu.edu/etd

\author{
Recommended Citation \\ Foreman, Anne M., "Negative Reinforcement by Timeout from Avoidance: The Roles of Discriminative \\ Cues, Shock-Frequency Reduction, and Response-Effort Reduction" (2012). Graduate Theses, \\ Dissertations, and Problem Reports. 260. \\ https://researchrepository.wvu.edu/etd/260
}

This Dissertation is protected by copyright and/or related rights. It has been brought to you by the The Research Repository @ WVU with permission from the rights-holder(s). You are free to use this Dissertation in any way that is permitted by the copyright and related rights legislation that applies to your use. For other uses you must obtain permission from the rights-holder(s) directly, unless additional rights are indicated by a Creative Commons license in the record and/ or on the work itself. This Dissertation has been accepted for inclusion in WVU Graduate Theses, Dissertations, and Problem Reports collection by an authorized administrator of The Research Repository @ WVU.

For more information, please contact researchrepository@mail.wvu.edu. 
Negative Reinforcement by Timeout from Avoidance: The Roles of Discriminative Cues, Shock-Frequency Reduction, and Response-Effort Reduction

Anne M. Foreman

\author{
Dissertation submitted to the \\ Eberly College of Arts and Sciences \\ at West Virginia University \\ in partial fulfillment of the requirements \\ for the degree of
}

Doctor of Philosophy

in

Psychology
Michael Perone, Ph.D., Chair
Karen Anderson, Ph.D.
Elizabeth Kyonka, Ph.D.
Tracy Morris, Ph.D.
Bernard Schreurs, Ph.D.

Department of Psychology

Morgantown, West Virginia

2012

Keywords: aversive control; timeout from avoidance; negative reinforcement

Copyright 2012 Anne Foreman 


\begin{abstract}
Negative Reinforcement by Timeout from Avoidance: The Roles of Discriminative Cues, Shock-Frequency Reduction, and Response-Effort Reduction
\end{abstract}

Anne M. Foreman

Timeout from avoidance is an effective reinforcer, but the reason is not firmly established. Rats responded on concurrent schedules of avoidance and timeout. Pressing the right lever postponed shocks according to a schedule with a response-shock interval of $30 \mathrm{~s}$ and a shock-shock interval of $5 \mathrm{~s}$, and pressing the left lever produced 2-min timeouts according to a variable-interval 45-s schedule. To assess the roles of shock-frequency reduction, response-effort reduction, and signals in varying the reinforcing efficacy of timeout, three experiments were conducted. In Experiment 1, to assess the point at which the reinforcing efficacy of the timeouts would be degraded, the probability of a timeout with shock was increased across conditions. Lower probabilities of shock in timeouts led to relatively small decreases in timeout responding for two of the rats and the maintenance of timeout responding for one rat, while higher probabilities led to more substantial decreases. In Experiment 2, the potential discriminative function of the temporal locations of shock in a yoking procedure from a previous study was investigated in two yoking conditions. In the Local-Yoking condition, there was no short-term change in the rate of shocks from time-in to timeout. The number and temporal location of shocks in the 2-min timeout duplicated the number and temporal location of shocks in the 2 min of time-in preceding the timeout. In the Random-Local-Yoking condition, the number of shocks in timeout duplicated the number of shocks in time-in, but the shocks were delivered in a random temporal position in the timeouts. Timeout responding was maintained at baseline levels for most of the rats in both conditions. In Experiment 3, the role of signals before shocks in restoring the reinforcing efficacy of timeout was investigated. After timeout responding was degraded in Experiment 1, in the subsequent condition, tones were added before the shocks in timeout. The addition of signals before shocks in timeout restored the reinforcing efficacy of the timeouts. The results from the present study suggest that multiple factors may be responsible for the reinforcing efficacy of timeout. 
I would like to thank the members of my committee - Mike Perone, Karen Anderson, Liz Kyonka, Tracy Morris, and Bernard Schreurs - for their helpful suggestions in the proposal phase and their thoughtful comments during the defense. I thank Leigh Schrimpf for her friendship and unfailing support during the first four years of graduate school. I thank Christian White and Sean Driscoll for helping me conduct daily sessions. I thank my family for their encouragement and positive reinforcement. I thank my brother Jimmy for making those lastminute trips to Morgantown to cheer me up during the rough patches, my mother for the daily phone calls, and my father for always believing in my capabilities - even when I didn't. I thank all of the service dogs that allowed me to exploit them as personal therapy dogs during the dissertation process. I thank Derek Clark for his love and support (and for his willingness to plan his life around my rat running schedule). I thank my advisor, Mike Perone, for seeing my potential as an undergraduate and offering me the opportunity to work in his lab. I am grateful for the time he spent shaping my thinking and my writing. I am proud to be a Peronian. 


\section{Table of Contents}

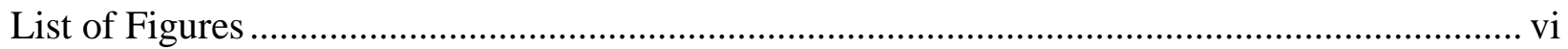

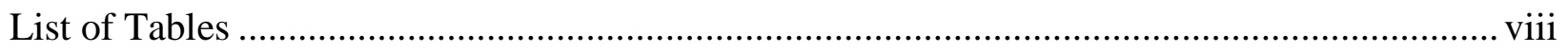

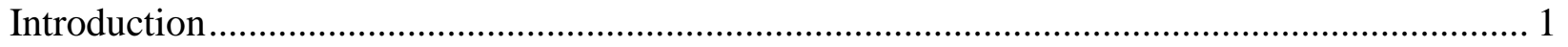

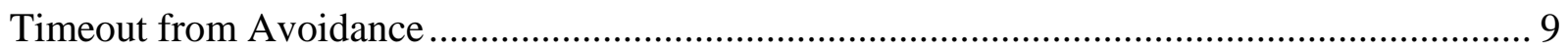

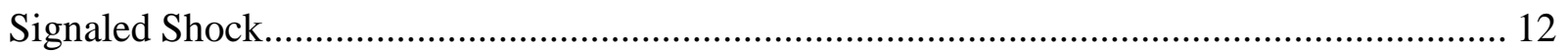

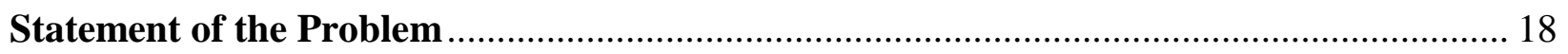

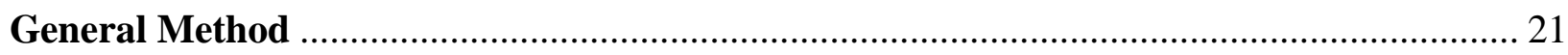

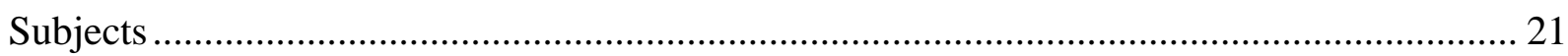

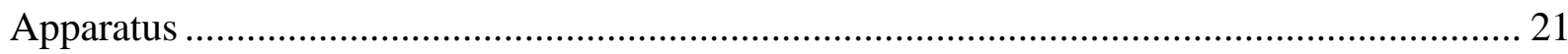

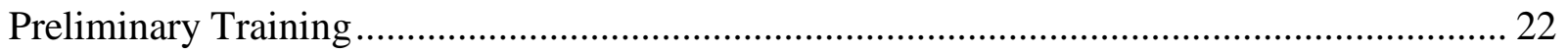

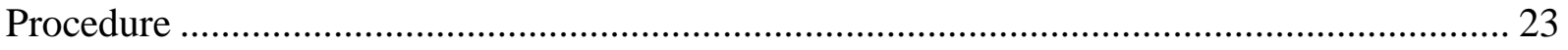

Stability Criteria

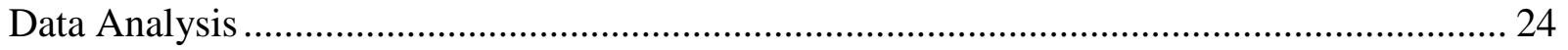

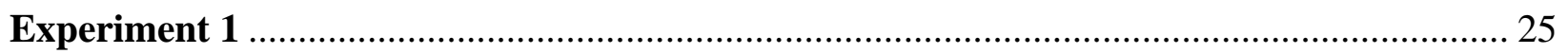

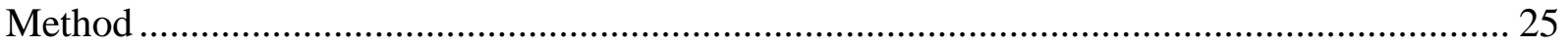

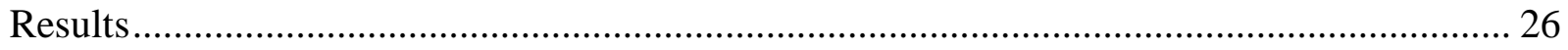

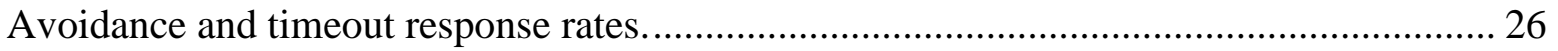

Shock-frequency reduction. .............................................................................................. 27

Response-effort reduction....................................................................................... 29

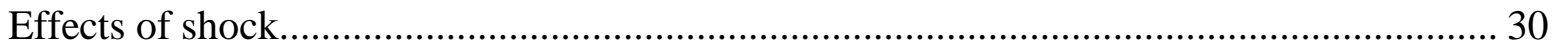

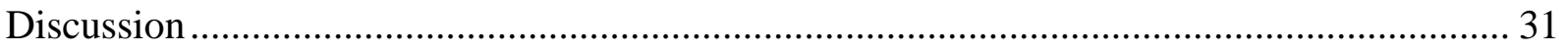

Experiment 2 ...

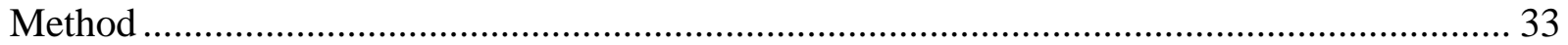

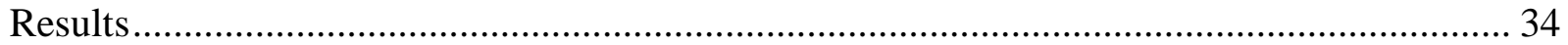

Avoidance and timeout response rates........................................................................ 34

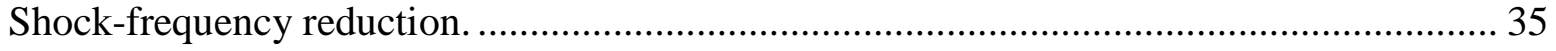

Response-effort reduction......................................................................................... 35

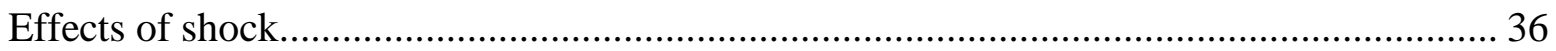

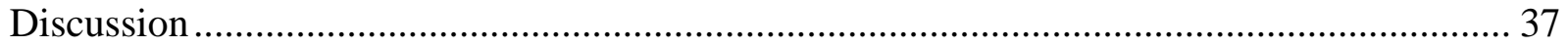

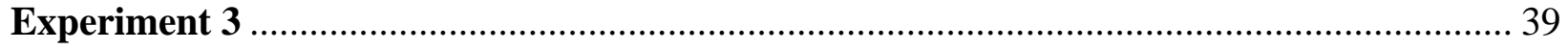

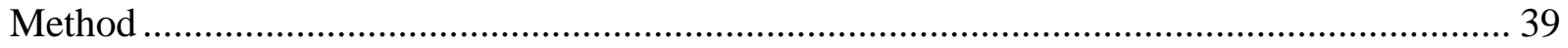

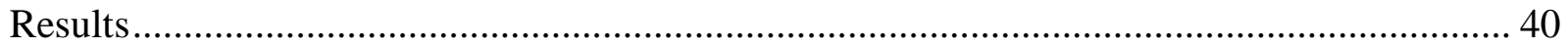

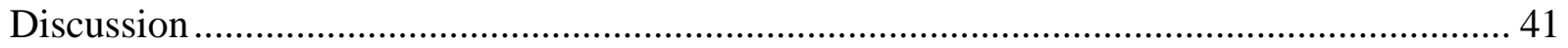




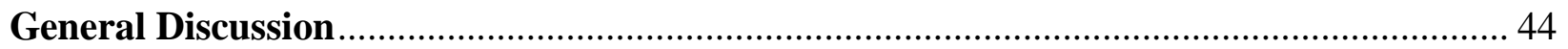

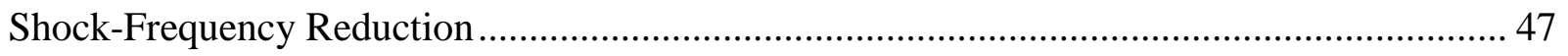

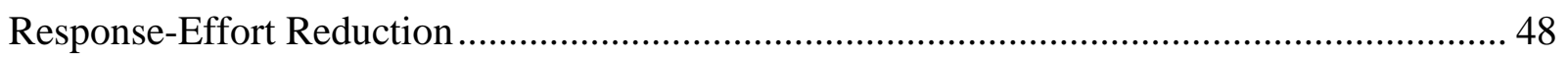

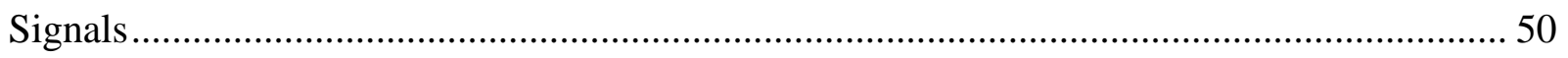

The Significance of Signaled vs. Unsignaled Aversive Events .......................................... 54

Timeout from Avoidance as an Animal Model of Phobic Behavior ..................................... 58

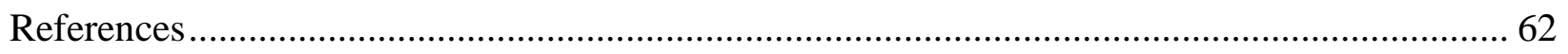




\section{List of Figures}

Figure 1. From Foreman (2009). Mean timeout response rates from the stable sessions of each condition. Error bars represent one standard deviation.

Figure 2. From Foreman (2009) Mean shock-frequency reduction (the timeout shock rate subtracted from the time-in shock rate) of the last six sessions of each condition. Error bars represent one standard deviation.

Figure 3. From Foreman (2009). Cumulative shocks during time-in (dotted line) and timeout (sold line) across the first 200 consecutive timeouts in Local Yoking (left column) and Molar Yoking (right column).

Figure 4. Experiment 1. Mean timeout responses per min for the last 10 sessions of each condition. Error bars represent one standard deviation. The dotted line shows $50 \%$ of baseline responding. Note the different axis for Rat AF5.

Figure 5. Experiment 1. Timeout responding plotted against shock- frequency reduction. Each data point represents performance in one of the last 10 sessions of each condition. Shockfrequency reduction values were calculated by subtracting the mean timeout shock rate for a session from the mean time-in shock rate for that session. Note the different axis for Rat AF5. 78

Figure 6. Experiment 1. Timeout responding plotted against response-effort reduction. Each data point represents performance in one of the last 10 sessions of each condition. Response-effort reduction values were calculated by subtracting the mean timeout avoidance response rate for a session from the mean time-in avoidance response rate for that session. Note the different axis for Rat AF5.

Figure 7. Experiment 1. Mean timeout responding plotted against the first timeout with shock in a session. Each data point represents mean performance in one of the last 10 sessions of each condition. If there were no shocks in a session, the data point was placed at 80 on the $\mathrm{x}$-axis (see explanation in text).

Figure 8. Experiment 1. Median intterresponse times on the timeout (left column) and avoidance (right column) levers for timeouts without shock (open circles) and with shock (filled circles). Data are from the last 10 sessions of each condition. Error bars represent the interquartile ranges.

Figure 9. Experiment 2. Mean timeout responses per min for the last 10 sessions of each condition. Error bars represent one standard deviation. Note the different axes.

Figure 10. Experiment 2. Timeout responding plotted against shock-frequency reduction. Each data point represents performance in one of the last 10 sessions of each condition. Shockfrequency reduction values were calculated by subtracting the mean timeout shock rate for a session from the mean time-in shock rate for that session......

Figure 11. Experiment 2. Timeout responding plotted against response-effort reduction. Each data point represents performance in one of the last 10 sessions of each condition. Response- 
effort reduction values were calculated by subtracting the mean timeout avoidance response rate for a session from the mean time-in avoidance response rate for that session. 84

Figure 12. Experiment 2. Median interresponse times (IRTs) on the avoidance (right column) and timeout (left column) levers in each condition for responses following timeouts with shock and timeouts without shock. The IRTs from each of the last 10 sessions of each condition were pooled. Error bars represent the interquartile ranges.

Figure 13. Experiment 2. Median post-shock response latency in the Shock-free (F) baseline (open circles) and in the two yoking conditions: Local Yoking (L) and Random Local Yoking (R) (filled circles) conditions. Data are from the last 10 sessions of each condition, and each data point represents the aggregated replications of the conditions. Error bars represent the interquartile ranges. 86

Figure 14. Data from Foreman (2009). Median post-shock response latency in the Shock-free (F) baseline and Molar-Yoking (M) (closed circles), and Local-Yoking (L) (open circles) conditions. Data are from the stable sessions of each condition. Error bars represent the interquartile ranges.

Figure 15. Experiment 3. Mean timeout responses per min for the last 10 sessions of the unsignaled (U) and signaled (S) conditions. The mean timeout response rates in the Shock-Free (SF) baseline of Experiment 1 are also included. Error bars represent one standard deviation. .. 88

Figure 16. Experiment 3. Timeout responding plotted against shock-frequency reduction. Each data point represents performance in one of the last 10 sessions of each condition. Shockfrequency reduction values were calculated by subtracting the mean timeout shock rate for a session from the mean time-in shock rate for that session. Note the different X-axis for Rat AF12.

Figure 17. Experiment 3. Timeout responding plotted against response-effort reduction. Each data point represents performance in one of the last 10 sessions of each condition. Response effort reduction values were calculated by subtracting the mean timeout avoidance response rate for a session from the mean time-in avoidance response rate for that session.

Figure 18. Experiment 3. Median interresponse times (IRTs) on the timeout (left column) and avoidance (right column) levers in each condition for responses following timeouts with shock and timeouts without shock. The IRTs from each of the last 10 sessions of each condition were pooled. Error bars represent the interquartile ranges. 


\section{List of Tables}

Table 1. Experiment 1. The programmed probability of a timeout with shock, the actual number of timeouts without and with shock, and the obtained probability of timeouts with shock for the last 10 sessions of each condition.

Table 2. Experiment 1. Summary of conditions, shock rates, avoidance response rates, discrimination indices ([avoidance responses in time-in and timeout / avoidance responses in time-in] x 100), and percentage of RS (response-shock) shocks avoided. Results are means of the last 10 stable sessions. Standard deviations are in parentheses........................... 70

Table 3. Experiment 2. Summary of conditions (shock free, local yoking, and random (R.) local yoking), shock rates, response rates, discrimination (discr.) indices (avoidance responses in timein / avoidance responses in time-in + avoidance responses in timeout), and percentage of RS (response-shock) shocks avoided. Results are means of the last 10 stable sessions. Standard deviations are in parentheses....................................................

Table 4. Experiment 3. Summary of conditions, shock rates, response rates, discrimination indices ([avoidance responses in time-in / avoidance responses in time-in + avoidance responses in timeout] x 100), and the percentage of RS shocks avoided. Results are means of the last 10 stable sessions. Standard deviations are in parentheses................................ 73 
Negative Reinforcement by Timeout from Avoidance: The Roles of Discriminative Cues, ShockFrequency Reduction, and Response-Effort Reduction

The domain of aversive control - negative reinforcement and punishment - has received renewed attention in applied behavior analysis (Iwata, 1987; Lerman \& Vorndran, 2002) and in clinical psychology with regard to behavioral models of depression (Abreu \& Santos, 2008; Kanter, Busch, Weeks, \& Landes, 2008) and post-traumatic stress disorder (Foa, Zinbarg, \& Rothbaum, 1992). While there is increased interest in aversive control in applied areas, basic research in aversive control has been scarce since the 1970s (Baron \& Perone, 2001). Aversive control is a pervasive feature of the environment (Skinner, 1953), and basic research in this area can only enhance the understanding and application of these behavioral processes outside of the laboratory.

Negative reinforcement is defined as the strengthening of behavior through the termination, prevention, or postponement of an aversive stimulus. One recently studied negative reinforcer is timeout from avoidance, which has been investigated with a procedure developed Verhave (1962) and refined by Perone and Galizio (1987).

Perone and Galizio's (1987) procedure arranged concurrent schedules of avoidance and timeout. Each schedule was correlated with its own response lever. By pressing the right lever, rats postponed electric foot shock according to a free-operant avoidance schedule (Sidman, 1953), and pressing the left lever occasionally produced 2-min timeouts. Differential stimuli were correlated with time-in and timeout. During time-in, both levers were inserted into the rat's chamber, a houselight illuminated the chamber, and a speaker delivered white noise. During a timeout, the timeout lever (and, in some experiments, also the avoidance lever) was retracted, the houselight and white noise were turned off, and the 
avoidance and timeout schedules were suspended. With this particular arrangement, timeout from avoidance has proven to be an effective reinforcer, maintaining behavior on variableinterval (VI) (Courtney \& Perone, 1992; Galizio \& Liborio, 1995; Perone \& Galizio, 1987), variable-ratio (VR) (Galizio, Hale, Liborio, \& Miller, 1993; Galizio \& Liborio, 1995), and progressive-ratio (PR) (Posner \& Baron, 1981) schedules, as well as multiple VI VI and multiple VI extinction schedules (Perone \& Galizio, 1987).

Other successful variations of the timeout procedure can be found in the literature. Earlier research maintained behavior on timeout procedures with rhesus monkeys (Sidman, 1962) and a chimpanzee (Findley \& Ames, 1965) on fixed-ratio (FR) and fixed-interval (FI) schedules, respectively. Other research has examined timeout from avoidance with conjoint schedules of avoidance and timeout (Baron, DeWaard, \& Lipson, 1977; Baron \& Trenholme, 1971).

As a method for studying negative reinforcement, the timeout procedure has several advantages over traditional avoidance-only procedures. A signaled timeout from avoidance has a distinct locus in time and can be scheduled in the same way as typical positive reinforcers such as food. Additionally, in avoidance-only procedures the motivating or establishing operations for avoidance responding and the reinforcement for responding are both manipulated by way of the rate or intensity of the shocks. In the timeout procedure, however, the confounding of these variables is circumvented; the establishing operations -- the avoidance parameters -- can be manipulated separately from the consequence -- the timeout parameters.

Although timeout has proven to be an effective reinforcer, the factors that underlie its efficacy remain to be decided. The production of a timeout involves three distinct changes in the environment. From time-in to the timeout, the stimuli associated with the avoidance contingency are removed, the frequency of shocks is reduced, and the response requirements on both levers 
are suspended for the duration of the timeout. Any of these factors, alone or in combination, could conceivably establish timeout as a reinforcer. Researchers have been concerned mainly with response-effort reduction and shock-frequency reduction. Response-effort reduction may play a dominant role in maintaining timeout responding because there is typically a large decrease in responding from time-in, in which the rat is responding on both the timeout and avoidance levers, to timeout, in which no responding typically occurs. Shock-frequency reduction cannot be completely ruled out, because in the typical procedure the production of a timeout always is associated with a reduction of shocks. The fact that shock-frequency reduction is implicated in a major theory of negative reinforcement - the single-factor theory of avoidance (Herrnstein \& Hineline, 1966) - increases interest in this factor.

To evaluate the role of shock-frequency reduction, Foreman (2009) degraded the amount of shock-frequency reduction afforded by a timeout by delivering shocks during timeouts in some of the conditions. In the Shock-Free baselines, no shocks were delivered in timeouts. In the Local-Yoking condition, the temporal sequence of shocks that occurred in each 2-min timeout was the same as in the 2 min of time-in preceding a timeout. This manipulation ensured that the local shock frequency was the same from time-in to timeout. In the MolarYoking condition, the temporal sequence of shocks during time-in in the previous session was played back during the subsequent session's timeouts. The goal of this condition was to keep the overall rate of shocks the same in time-in and timeout. Figure 1 shows mean response rates on the timeout lever in the stable sessions of each condition for each rat (there is no baseline preceding AF21's Molar- Yoking condition; see Foreman (2009) for an explanation). The means are based on data aggregated across replications of each condition. Rate of responding on the timeout lever is accepted as a measure of the reinforcing efficacy of the consequence of 
that responding, i.e., of the timeout. In Local Yoking, timeout responding was maintained near baseline levels for three of the rats. Responding was reduced from baseline levels, but not eliminated, for the remaining rat (AF20). In Molar Yoking, timeout responding increased from baseline levels for one rat (AF15), and for the other rats, responding in this condition was reduced below the levels obtained in both the Shock-Free baseline and Local-Yoking conditions.

The difference in timeout responding across the two yoking conditions was unexpected, as the conditions were construed merely as functionally equivalent experimental operations for eliminating shock-frequency reduction in timeout. The differences could have resulted from three factors. First, did the differences in shock rates in time-in between the two conditions affect the reinforcing value of the timeouts in the two conditions? If more shocks were delivered in time-in in one yoking condition, then more shocks also would be delivered in timeout in that condition, thereby reducing the value of the timeout. This was not the case, however. There were no systematic differences in overall shock rates between the two yoking conditions. In general, shock rates were under 0.10 shocks per min. It is unlikely that differences in overall shock rates in time-in were responsible for the reduced timeout response rates in the Molar-Yoking condition.

Second, was the difference in timeout responding obtained because there were differences in the success of the yoking procedures in eliminating shock-frequency reduction? If shock-frequency reduction was not eliminated in one yoking condition, there could have been greater decreases in the overall rate of shock from time-in to timeout in that condition. If there were greater decreases in the overall rate of shock from time-in to timeout in one condition, than there may have been higher rates of timeout responding because the production 
of a timeout afforded greater shock-frequency reduction. Alternately, if there were increases in shock frequency from time-in to timeout in one condition, then the reinforcing efficacy of the timeouts may have been reduced leading to decreased rates of timeout responding in that condition. To assess whether an overall reduction or an overall increase in shocks from time-in to timeout was responsible for the different results, Figure 2 shows the mean shock-frequency reduction from the stable sessions of each condition for each rat. If shock-frequency reduction is positive, then there was an overall decrease in shocks from time-in to timeout, and if shockfrequency reduction is negative, then there was an overall increase in shocks from time-in to timeout. For most of the rats, shock-frequency reduction was low and positive across conditions. The shock rates during time-in for Rat AF20 were much higher than for the other rats in the Shock-Free baseline conditions, therefore timeouts afforded a greater reduction in shock frequency. For this rat, shock-frequency reduction was negative in the yoking conditions; overall shock rates increased from time-in to timeout. Rat AF20 also had the lowest rates of timeout responding in the yoking conditions of all of the rats, and it is possible that this increase in shocks from time-in to timeout may have reduced the reinforcing value of the timeouts. For most of the rats, the yoking conditions were successful at eliminating overall shock-frequency reduction, and in the cases in which they were not successful, shockfrequency reduction was minimal and undifferentiated between the yoking conditions. Therefore, differences in overall shock-frequency reduction between the two conditions were not responsible for the differences in responding. For Rat AF20, the yoking conditions were not successful at eliminating overall shock-frequency reduction, and indeed, in the yoking conditions, there were increases in shock frequency from time-in to timeout. These increases in shock may have devalued timeout as a reinforcer. 
The reasons for the obtained differences in timeout responding were not evident upon examination of the overall measures of shock rates and shock-frequency reduction between the two yoking conditions. These steady-state measures may not have revealed the variables responsible for the obtained differences. Therefore, the third factor that could be responsible for the differences in timeout responding may be found in the subjects' initial exposure to shocked timeouts in the yoking conditions. Did differences in the shock rates in time-in and timeout in the first few sessions of the yoking conditions produce the differences in timeout responding? To answer this question, the frequency of shocks in time-in and timeout in the first few sessions of each yoking condition was examined. Figure 3 shows the cumulative shocks in the 2-min of time-in preceding each timeout and the cumulative shocks in the subsequent timeouts across the first 200 timeouts (approximately 4 to 5 sessions). The dotted line represents shocks in time-in and the solid line represents shocks in timeout. In Local Yoking (right column), only one line is visible because the number of shocks in timeout and the preceding 2-min of time-in were identical. This indicates that the manipulation was successful at eliminating local shockfrequency reduction. In Molar Yoking, however, local shock-frequency reduction was not eliminated. For three of the four rats, more shocks were delivered in timeout than in time-in. These local increases in shock frequency from time-in to timeout may have reduced the reinforcing value of timeout, leading to the lower rates of timeout responding observed in the stable sessions of the Molar-Yoking conditions. Therefore, the obtained differences in the yoking conditions may have resulted from the differential local shock-frequency changes from time-in to timeout in the initial exposure to these conditions.

These results contribute to the research suggesting that shock-frequency reduction is not solely responsible for timeout responding. In the Local-Yoking condition, when local shock- 
frequency reduction from time-in to timeout was eliminated, responding to produce timeouts was maintained. In the Molar-Yoking condition, for one rat, when the manipulation was successful at eliminating overall shock-frequency reduction, timeout responding was maintained. For the other rats in the Molar-Yoking condition, local shock frequency often increased from time-in to timeout, and timeout responding was reduced for most rats but did not extinguish.

The present experiments were designed to attempt to elucidate the factors that may have contributed to the maintenance or reduction of responding in the yoking conditions in Foreman's (2009) experiment. Adding shocks to timeouts may decrease the reinforcing efficacy of the timeouts, but it is unclear at what point the reinforcing efficacy is degraded to override the reinforcing value of response-effort reduction afforded by the timeouts and produce decreases in timeout responding. For many of the rats, a sudden increase in shocks in timeout (from no shocks in the preceding baseline to potentially many shocks in multiple timeouts) occurred within the first few sessions of the Molar-Yoking condition, so it is possible that timeout responding could be maintained when an intermediate frequency of shocks occur in the timeouts. In Experiment 1, shocks were delivered in an increasing proportion of timeouts across conditions. This manipulation was intended to permit an analysis of the effects of shocks in timeouts at intermediate frequencies.

The disparate results obtained between the two yoking conditions in Foreman (2009) may have been obtained because of the specific differences in the method of yoking the shocks. In Local Yoking, the shocks just received in time-in were delivered in the same temporal sequence in the following timeout. Because of its recency, a shock delivered in timein may function as a discriminative cue for shocks that will be delivered in timeout. There was 
no such discriminative relation between shocks in time-in and timeout in Molar Yoking; the temporal sequence of shocks in timeouts came from the sequence during time-in in the previous day's session. This presence of a discriminative cue in Local Yoking and the absence of a discriminative cue in Molar Yoking may have contributed to the differences in timeout responding obtained in the two conditions. This line of reasoning is supported by the literature on comparisons of shocks preceded by signals (e.g., tone or buzzer) and shocks that are not preceded by such stimuli. In certain arrangements, shocks preceded by signals have been found to be less aversive than shocks with no such stimuli preceding them (e.g., Badia \& Culbertson, 1972). Therefore, if the shocks in time-in functioned as a discriminative cue for shocks delivered in timeout in Local Yoking, then the shocks delivered in the timeouts in Local Yoking may have been less aversive then the shocks delivered in timeout in Molar Yoking. Experiment 2 investigated this potential discriminative function of shocks in Local Yoking. If the temporal sequence of shocks during time-in facilitated discrimination of the sequence of shocks in the upcoming timeout, thus reducing their aversiveness, then disrupting the temporal sequence of shocks should eliminate this function and restore the aversiveness of shocks in timeout. In Experiment 2, the temporal sequence of shocks in the 2-min of time-in preceding a timeout was randomized and played back in the timeout. For example, if a shock was to be delivered $30 \mathrm{~s}$ before the end of the timeout, it was instead delivered at a random second between 1 and 120 during the timeout. If the shocks in timeout were more aversive with this manipulation, then timeout responding should have been reduced when compared to responding in a Local-Yoking condition in which the temporal sequence of shocks from timein to timeout was preserved.

In Experiment 3, the role of discriminative cues in reducing the aversiveness of shock was investigated further by adding a discriminative stimulus before shocks delivered in 
timeout. After Experiment 1 identified the probability of timeouts with shock sufficient to reduce timeout responding, a tone was played before each shock delivered in timeout. If adding this discriminative stimulus before shocks led to a recovery in timeout responding, then it would support the hypothesis that discriminability affects the aversiveness of shocks delivered in timeout. These results would suggest that the differences in the results obtained with the two yoking conditions in Foreman (2009) may have been attributable to differences in the aversiveness of shocks in timeout between the two conditions.

A comprehensive explanation of the rationale for the series of experiments proposed in the present study requires a review of two lines of research. The first section will describe the research that has been conducted investigating the factors that potentially underlie the reinforcing efficacy of timeout. Because Experiments 2 and 3 are concerned with the potential discriminative role of yoked shocks and explicit cues in decreasing the aversiveness of shocks, the final section will consider relevant research on the potential role of discriminative stimuli in modulating the aversive function of shock.

\section{Timeout from Avoidance}

Timeout has been demonstrated to be an effective reinforcer, but as mentioned above, there has been debate about which variables maintain timeout responding. Experiments have been conducted examining the roles of the three events that occur when a timeout is produced: the changes in stimuli, the reduction in shocks, and the reduction in response effort.

Perone and Galizio (1987) ruled out stimulus change as a possible variable maintaining timeout responding. In the second experiment of their study, rats pressed levers on concurrent schedules of avoidance and timeout. A shock-postponement schedule (Sidman, 
1953) was programmed on the avoidance lever. In the absence of responding, shocks were delivered according to the shock-shock (SS) interval. Each response postponed the next shock according to the response-shock (RS) interval. In their study, the SS interval was $5 \mathrm{~s}$ and the RS interval was $30 \mathrm{~s}$. The schedule on the timeout lever differed across conditions. In the baseline condition, responses on the timeout lever produced 2-min timeouts according to a variable-interval 45-s (VI 45-s) schedule. In the experimental condition, responses produced 2-min "sham-timeouts" according to the VI schedule: the stimulus changes that accompanied a timeout occurred as usual, but the shock-postponement contingency remained in effect. Shocks were delivered during the sham-timeouts in this condition unless the rat pressed the avoidance lever to postpone them. The rats responded to avoid shocks during the sham-timeouts, albeit less effectively than during time-in. Responding on the timeout lever extinguished in the sham-timeout condition, demonstrating that the stimulus changes that accompanied the timeout period were not maintaining responding on the timeout lever.

Another variable that may reinforce timeout responding is the reduction in the frequency of shocks afforded by the suspension of the avoidance contingency during a timeout. Research has suggested that timeout responding may not be sensitive to shock-frequency reduction. Courtney and Perone (1992) programmed concurrent schedules of avoidance and timeout. On the avoidance lever, rats avoided shocks on a variable-cycle (VC) shock-deletion schedule. The VC shock-deletion schedule programmed shocks at irregular intervals (or cycles). In each cycle, pressing the lever cancelled the shock that would otherwise have been delivered at the end of that cycle, and additional responses had no programmed consequences. A multiple schedule was programmed within this avoidance schedule. The components of the multiple schedule differed in terms of programmed shock rate during time-in and were signaled by a constantly 
illuminated houselight or a flashing houselight. For example, in the first condition, a VC 30-s schedule was programmed in one component of the multiple schedule and was signaled by a flashing houselight, and a VC 60-s schedule was programmed in the other component and was signaled by a constantly illuminated houselight. Within a session, each component lasted 5 min (not counting timeouts) and the two types of components alternated until each was presented five times. In subsequent conditions, the parameters of the VC schedules were manipulated in both components of the multiple schedule to obtain a range of shock rates and avoidance response rates. Throughout all conditions, responding on the timeout lever produced 2-min timeouts on a VI 45-s schedule. Analyses based on the generalized matching law (Baum, 1974) were conducted to assess the sensitivity of timeout responding to shock-frequency reduction and reductions in response effort. Responding on the timeout lever was relatively insensitive to received or scheduled reductions in shock frequency.

These results support the findings of Perone and Galizio (1987). Received shock rates in their study were usually less than 0.3 shocks per min, and in several cases were below 0.05 . Because these rates were much lower than those of studies that demonstrated the reinforcing efficacy of shock-frequency reduction (e.g., reductions of about 0.6 to 3.6 shocks per min: de Villiers, 1974; 3 to 9 shocks per min: Herrnstein \& Hineline, 1966), Perone and Galizio argued that it was unlikely that the reduction in shock frequency afforded by the timeouts was maintaining responding. Perone and Crawford (1992) made a similar argument. In their study of timeout from avoidance, received shock rates varied among rats. One rat's shock rate was always below 0.1 shocks per min. Two rats contacted shocks at rates 5 to 20 times higher, but their response rates on the timeout lever did not exceed the rates of the rat proficient at avoiding shocks. Perone and Crawford pointed out that if the reinforcing efficacy of timeout was based 
on shock-frequency reduction, one would expect to see a relation between rates of received shock in time-in and responding on the timeout lever. In their study, this relation was not evident despite the wide range of received shock rates.

In Courtney and Perone's (1992) study, there was evidence that responding on the timeout lever was sensitive to changes in the rates of concurrent responding on the avoidance lever. Producing a timeout suspends the response requirement on the avoidance lever for the duration of the timeout. As the response rate on the avoidance lever increased during time-in, the production of a timeout led to greater reductions in response effort from time-in to a timeout. Two of the rats showed a high degree of sensitivity to response reduction, even though they were not sensitive to changes in shock frequency. Perone and Crawford (1999) offered an explanation. They noted that of the two events that are interrupted by timeout, avoidance responding and the delivery of shocks, avoidance response rates were far more frequent (7 to 147 times higher in their study). Given this difference, the large reduction responding from time-in to the timeout would be more salient than the rather small reduction in shock frequency.

In summary, timeout has been demonstrated to be an effective reinforcer, but the reasons underlying its reinforcing efficacy are unknown. Previous studies have ruled out stimulus change as a reinforcer, cast doubt on a singular role for shock-frequency reduction, and provided preliminary support for a role for the response-effort reduction obtained when the need for avoidance responding is suspended during a timeout.

\section{Signaled Shock}

The environmental context has been shown to play an important role in altering the aversiveness of stimuli such as electric foot shock. Adding a stimulus (e.g. light or tone) before the delivery of electric shocks can reduce the aversiveness of those shocks. This has been 
established in research demonstrating that rats prefer environments with signaled shock over environments with unsignaled shocks. Knapp, Kause, and Perkins (1959) conducted one of the first demonstrations of this preference. In their discrete-trial procedure, rats ran down a Tshaped maze into either of two arms. At the end of each arm was a delay box. When the rat entered the delay box, a door closed, and the rat was confined for $45 \mathrm{~s}$. After this short delay, the door was opened to a connecting goal box in which food was available. The rats could run to either arm of the maze in each trial, and several trials were conducted each day. After 5 days of training, a 0.7-s (60-v) shock was delivered in each delay box. After the shock was delivered, the door to the goal box was opened. In one arm, the onset of four lights and a buzzer signaled the shock. In the other arm, the lights and buzzer were turned on after the shock was delivered. In most of the trials, all of the rats ran to the arm of the maze in which the lights and buzzer were turned on before the shock, that is, the arm in which the shocks were signaled.

Similar results were obtained by Lockard (1963), who used two connected chambers separated by a Plexiglas wall. The rat could move from one chamber to the other through a small door in the wall. The walls of one chamber had vertical stripes and the other had horizontal stripes. In each trial, a 2-s (0.28-mA) shock was delivered in both chambers simultaneously. For the experimental group, in one chamber the shock was always preceded by a signal (7-s blinking light), while in the other chamber the shock was unsignaled. For the control group, a 7-s blinking light was presented independently of shock delivery (it was not correlated with the onset of shock) in one chamber, and in the other chamber, the shock again was unsignaled. The rats remained in the apparatus for the entire session and could move from one chamber to the other throughout the session. The rats in the control group spent approximately equal amounts of time in the two chambers. The rats in the experimental group, 
however, spent more time in the chamber with signaled shock.

In the typical free-operant procedure to assess choice between signaled and unsignaled shock, the subjects are rats and the response is a lever press. The experimental session consists of two environmental contexts. In both contexts, electric shocks are delivered after fixed or variable intervals of time (depending on the experiment). In one context, the shocks are preceded by a stimulus, typically a tone (signaled-shock context), and in the other context, there are no programmed stimuli preceding shock (unsignaled-shock context). There are no other differences in stimuli between the two contexts. During training, the contexts are alternated at regular intervals, so that the rat is exposed to each equally often. After training, the session starts in one of the contexts (either signaled or unsignaled shock) and a response switches the session to the other context for a short period of time, typically $2 \mathrm{~min}$. After this period of time elapses, the original context is restored. The amount of session time spent in each context is used to measure preference.

Badia and Culbertson (1972) used such a procedure. Two-lever operant chambers were used. Shocks (2-s, 75-MW) were delivered after variable periods of time averaging 2 min (variable-time (VT) 2-min schedule). In the signaled context, a brief tone preceded shocks. A response on a lever changed the shock context from unsignaled to signaled for a short period of time. All of the rats pressed the lever to switch the shock context within the first three sessions, and each subject spent almost the entire session in the signaled context. The experimenters manipulated the type of shock, either escapable (a response on a second lever turned off an ongoing shock) or inescapable, between two experiments. Regardless of whether the shocks were escapable or not, the rats spent the majority of sessions in the signaled context. These results have been directly replicated (Lewis \& Gardner, 1977). 
Other studies using similar procedures have found preferences for a denser schedule of signaled shock over a leaner schedule of unsignaled shock (Badia, Coker, \& Harsh, 1973), longer signaled shocks over shorter unsignaled shocks, avoidable signaled shocks over avoidable unsignaled shocks, and higher-intensity signaled shocks over lower-intensity unsignaled shocks (Badia, Culbertson, \& Harsh, 1973). Lever presses in all of these studies switched the shock context from unsignaled to signaled, but rats also spent the majority of a session in the signaled context when a lever press changed the context from signaled to unsignaled (Badia, Culbertson, \& Lewis, 1971).

MacDonald and Baron (1973) assessed the relative aversiveness of signaled and unsignaled shock using chain schedules. In a chain schedule, the subject must complete the requirement for two (or more) simple schedules in a fixed sequence, and each schedule is accompanied by a different stimulus. In the initial link, pressing a lever produced the terminal link schedule after variable intervals of time averaging $90 \mathrm{~s}$ (VI 90-s schedule). In the terminal link, evaporated milk was delivered according to a VT 15-s schedule for 5 min. After baseline rates of responding were established, 2-s (0.2-mA) shocks were delivered in the terminal link according to a VT 4-min schedule. Two of these chain schedules were alternated within a session. In one chain schedule, the shocks delivered in the terminal links were signaled (by a 5s buzzer), and in the other chain schedule, the shocks were unsignaled. The dependent measure of interest was the rate of responding in the initial links of each schedule. The addition of shocks reduced responding in the initial links, indicating that the shocks degraded the reinforcing value of the terminal link. The two types of shock, however, produced differential reductions in initial-link responding: Rates were lower in the initial link leading to the terminal link with unsignaled shocks, indicating that the shocks decreased the reinforcing value of the 
terminal link to a greater extent than signaled shocks.

In addition to studies that assessed the relative aversiveness of different types of shock, several studies have demonstrated differential response suppression when signaled or unsignaled shocks are superimposed onto existing schedules of reinforcement. In Seligman's (1968) study, lever presses produced food deliveries after variable intervals averaging $1 \mathrm{~min}$ (VI 1-min schedule). After several baseline sessions, one group of rats received 3 responseindependent shocks (3-s, 0.88-mA) randomly interspersed within each session. For one group of rats, the shocks were preceded by a 1-min signal, and the type of signal was changed across conditions. The signals were a white lever light, a flickering green lever light, a tone, or a drop in the volume of the white noise (which was normally on during the session). For another group of rats, these signals were uncorrelated with shock delivery shocks could occur before, during, or after signal presentation. Greater suppression of responding from baseline rates occurred in the group that received signals that were uncorrelated with shock. Put another way, the signals reduced the suppressive function of the shocks.

Hymowitz (1973) evaluated the effects of signals on punishment, that is, responsedependent shock. Rats' lever presses were reinforced with food on a VI 35-s schedule. In one group of rats, responses also produced shock (0.5-s 0.4-mA) on a FI 65-s schedule. In the other group of rats, responses also produced shock on a VI 65-s schedule. The frequency of signals before the shocks was manipulated across groups. A 5-s signal, the illumination of three cue lights located above the lever, preceded either all of the shocks, none of the shocks, or a half of the shocks (in random order). The least amount of response suppression occurred when a signal always preceded a shock. Relatively more suppression of responding occurred 
when signals were never presented or were intermittently presented, regardless of whether the shocks occurred after regular (FI) or irregular (VI) intervals.

In a within-subject analysis of response suppression, Hymowitz (1976) arranged a compound schedule in which two schedule components alternated within an experimental session. In both components, a VI 35-s schedule of pellet delivery was arranged. After stable responding was achieved in both components, 0.5-s (0.4-mA) shocks were added. Shocks were delivered according to a VI 65-s schedule. In one component, the shocks were preceded by a brief signal, 5-s onset of a red cue light, and in the other component, no signal preceded the shocks. The type of compound schedule, either multiple or mixed, was manipulated across conditions. In a multiple schedule, different stimuli are correlated with each component, and in a mixed schedule, the same stimulus is present during both components. In the mixed schedule conditions, there were no differential stimuli correlated with the presence of signaled or unsignaled shock; the chamber remained dark during both components or the houselight was on during both components. In the multiple schedule conditions, the chamber was dark during the component with unsignaled shock and the houselight was on during the component with signaled shock. Suppression was assessed by comparing the number of responses that were emitted in the shock-free baseline sessions to the sessions in which shocks were delivered. In the mixed schedule, responding was suppressed roughly equally in both components. In the multiple schedule, however, more response suppression occurred in the unsignaled-shock component. Signaled shocks produced less response suppression than unsignaled shock, but only when the type of shock that would be delivered was correlated with an external stimulus. The mixed-conditions were essentially the same as the condition in Hymowitz (1973) when shocks were preceded by signals $50 \%$ of the time, and similar results were obtained - 
responding was suppressed when shocks were only intermittently preceded by signals.

Hymowitz (1977) extended the research on response suppression and signaled shock to schedule-induced polydipsia. Schedule-induced polydipsia occurs when a food-deprived (but not water-deprived) rat drinks an excessive amount of water during a session (three to four times their usual daily intake) with bouts of drinking typically occurring after each pellet delivery (Falk, 1961). In Hymowitz's series of experiments, responding was reinforced on an FI 40-s schedule and water was freely available in the chamber through a metal spout. After 20 baseline sessions, 0.5 -s response-independent shocks were delivered on a VT 70-s schedule. The rats were exposed to signaled- and unsignaled-shock conditions. During the signaled conditions, the shocks were always preceded by a 10-s signal (two cue lights were illuminated). During the unsignaled conditions, no signals were presented before shocks. Greater suppression of lever pressing occurred in the sessions with unsignaled shock than in sessions with signaled shock, corroborating the results of previous studies. In addition, schedule-induced licking was suppressed to a greater degree in the unsignaled-shock conditions than in the signaled-shock conditions for most rats. This study extended the findings of differential response suppression with signaled and unsignaled shock to a behavior other than lever pressing.

In summary, studies have found that signaled shock is less aversive than unsignaled shock. Rats prefer signaled shock to unsignaled shock. In punishment procedures less response suppression occurs when schedules of signaled shock, rather than unsignaled shock, are superimposed over schedules of reinforcement.

\section{Statement of the Problem}


Timeout from avoidance has been demonstrated to be an effective reinforcer, but the factors underlying its reinforcing efficacy are open to debate. Previous research has ruled out stimulus change and has cast doubt on the role of shock-frequency reduction. In an experiment designed to eliminate the shock-frequency reduction associated with timeout, Foreman (2009) analyzed two methods to equate shock rates during time-in and timeout. The two methods yielded discrepant results. When the local rate of shock was the same from time-in to timeout (Local Yoking), the reinforcing efficacy of timeout was unaffected and responding was maintained. When the overall rate of shock from time-in to timeout was the same from session to session (Molar Yoking), the reinforcing efficacy of timeout was degraded and responding decreased (but it did not extinguish). The discrepant results were not due to differences in overall shock rates in time-in or differences in overall and local shock-frequency reduction. The data do suggest, however, that the initial exposure to the yoking conditions may have contributed to the differences in timeout responding. In the first few sessions of the Molar-Yoking conditions, more shocks were delivered in timeout than in time-in. These increased shocks in timeout relative to the shocks in time-in may have degraded the reinforcing value of the timeouts and lowered response rates on the timeout lever. Another possibility is that the shocks in the timeouts in Local Yoking may have been less aversive than the shocks in Molar Yoking. Because any shocks occurring shortly before a timeout were repeated during the timeout in Local Yoking, the time-in shocks may have served as signals for the timeout shocks. This signaling function may have reduced the aversiveness of the shocks delivered in the timeouts.

The purpose of the present set of experiments was to investigate the factors that may have contributed to the discrepant results obtained in Foreman (2009). Experiment 1 investigated the persistence of timeout responding when the probability of a shock in timeout was raised across 
conditions. The idea was to eliminate the uncontrolled, sudden increase in shocks during timeouts that occurred in the previous study, permitting the analysis of timeout responding when the rate of shock deliveries was under direct experimental control rather than yoked to the rat's behavior. By raising the probability of a shock in timeout across conditions, Experiment 1 was designed to assess the point at which the reinforcing efficacy of timeout is sufficiently degraded to produce decreases in timeout responding.

In the Local-Yoking condition of Foreman's (2009) experiment, the temporal sequence of shocks in the 2-min of time-in preceding a timeout were played back in the identical temporal sequence during the timeout. It is possible that the shocks received in time-in may have functioned as signals for upcoming shocks in timeout, potentially attenuating the aversiveness of the shocks in timeout. Research has demonstrated that adding signals before shock can reduce the aversiveness of the shocks. It is unclear whether the temporal sequence of shock or just the mere occurrences of shocks may have served as a signal. Experiment 2 of the study investigated this empirical question. Two types of Local Yoking were arranged. One directly replicated the Local-Yoking procedure in Foreman (2009). By comparison, in the Random Local Yoking condition, shocks in the 2-min preceding a timeout were played back in the following timeout, but in a random arrangement. If one shock was scheduled to be delivered in the timeout, a number between 1 and 120 was selected by the computer program at random. The shock was then delivered at that second during the timeout. For example, if 5 were the randomly selected number, then the shock would be delivered $5 \mathrm{~s}$ into the timeout. There were no constraints on the selection of the number between 1 and 120; the shock could have been delivered at any point from the first second to the last second of the 2-min timeout. If the temporal sequence of shock 
was one variable maintaining timeout responding in Local Yoking, then it was expected that timeout responding would decrease relative to a standard Local-Yoking condition.

Experiment 3 evaluated the effects of adding explicit signals to shocks during timeouts. In Foreman's (2009) study, the Molar-Yoking procedure decreased timeout responding from the Shock-Free baseline levels. One possible reason may have been the absence of signals for the shocks delivered in the timeouts in Molar Yoking. Unlike Local Yoking, the sequence of shocks in the timeouts in Molar Yoking was not based on the rat's behavior in the preceding 2-min of time-in, but rather from the rat's behavior during time-in in the previous session. Therefore, the shocks in time-in during a session of Molar Yoking could not signal the shocks to be delivered in the timeout. Experiment 3 was implemented with the same rats as in Experiment 1. In Experiment 1, the probability of a timeout with shock was increased across conditions and ended when timeout responding was reduced by at least $50 \%$ of the Shock-Free baseline. In Experiment 3 , the condition that produced such reductions was replicated with the addition of a brief signal before the shocks delivered during the timeouts.

\section{General Method}

\section{Subjects}

Seven male Sprague-Dawley rats were housed in individual cages under a 12:12 hour reversed light/dark cycle with free access to food and water.

\section{Apparatus}

One custom-built operant chamber and two commercial chambers (Lehigh Valley Electronics) were used. The interiors were approximately $30 \mathrm{~cm}$ long, $21 \mathrm{~cm}$ high, and $19 \mathrm{~cm}$ deep. In each commercial chamber, the side walls and ceiling were constructed of Plexiglas, and the end walls of stainless steel. The floor consisted of stainless steel rods, $0.5 \mathrm{~cm}$ in diameter, 
spaced $1.9 \mathrm{~cm}$ apart, center to center. Illumination was provided by a $28-\mathrm{V}$ houselight (No. 1820) mounted behind a sheet of white paper on a side wall. Two levers were centered $10 \mathrm{~cm}$ apart on the front wall, $9 \mathrm{~cm}$ above the grid floor. In the custom-built chamber, the rear wall, ceiling, and one side wall were constructed of clear Plexiglas, the other side wall of stainless steel, and the front wall of aluminum. The levers were $8.5 \mathrm{~cm}$ apart, $9.6 \mathrm{~cm}$ above the floor, and the floor rods were spaced $1.7 \mathrm{~cm}$ apart. General illumination was provided by a houselight at the top of the front wall. In all four chambers, the left lever (BRS/LVE, RRL-015) was retractable and the right was fixed in place. The levers required a force of approximately $0.3 \mathrm{~N}$ to operate. Shock generators and scramblers (Med Associates, ENV-414) delivered scrambled 1-mA shocks lasting $0.5 \mathrm{~s}$ through the grid floors (but not the levers or walls). Each chamber was enclosed in a soundattenuating box equipped with a fan for ventilation and a speaker for white noise. Throughout the experiment, activation of the white noise generator and houselight signaled the onset of the session, and these events were terminated at the end of the session as well as during the timeout periods. Control and recording operations were accomplished with microcomputers running programs written in Visual Basic 6.

\section{Preliminary Training}

Following the procedure described by Perone and Crawford (1999), the rats were trained to press the right lever (avoidance lever) on a shock-postponement schedule with an RS interval of $30 \mathrm{~s}$ and a SS interval of $5 \mathrm{~s}$. The houselight and white noise was turned on and lever presses were followed by a feedback stimulus consisting of a 0.5-s offset of the white noise. This avoidance training was continued until the rats avoided at least $80 \%$ of the shocks arranged by the RS timer over the most recent 3 sessions. 
Next, a multiple schedule with alternating 10-min components of avoidance and timeout was implemented to facilitate discrimination of avoidance and timeout from avoidance. In the avoidance component, the houselight and the white noise were on and avoidance responses postponed shock as described above. In the timeout component, the houselight and the white noise were off, the shock-postponement schedule was suspended, and avoidance responses had no programmed consequences. As a correction procedure, the timeout component did not end unless at least $1 \mathrm{~min}$ had elapsed since the last response. Training on the multiple schedule continued until virtually all of the responses occurred during the avoidance component.

In the final phase of preliminary training, both levers were inserted. Responses on the avoidance lever postponed shocks, and responses on the left lever (timeout lever) produced 5min timeouts on an FR-1 schedule. During a timeout, the timeout lever was retracted, the houselight and white noise were turned off, the shock-postponement schedule was suspended, and avoidance responses had no programmed consequences. Over several sessions the timeout duration was reduced from 5 min to $2 \mathrm{~min}$, and the timeout schedule was leaned from FR 1 to VI 45 s (Fleshler \& Hoffman, 1962). The feedback for responses on the timeout lever was 0.5-s offset of the houselight.

\section{Procedure}

Sessions began with a 20-min avoidance-only warmup period in which the timeout lever was retracted, the houselight was turned off, the white noise was turned on, and the shockpostponement schedule was programmed on the avoidance lever. After the warmup, the session proper was signaled by the onset of the houselight and the insertion of the timeout lever. The session proper lasted until 60 min accumulated in time-in, regardless of the frequency of the timeouts. During time-in, both levers were inserted, and the houselight and the white noise were 
on. Pressing the avoidance lever postponed shock according to a schedule with a RS interval of $30 \mathrm{~s}$ and a SS interval of $5 \mathrm{~s}$, and pressing the timeout lever produced 2-min timeouts on a VI 45 s schedule. Sessions were conducted at least 6 days a week at approximately the same time every day.

\section{Stability Criteria}

Each condition lasted at least 20 sessions. Conditions were changed when response rates on the timeout lever were stable over 10 consecutive sessions. If the difference between the mean of the first 5 sessions and the mean of the last 5 sessions was within $15 \%$ of the 10 -session mean and visual inspection of the daily sessions revealed no systematic trends, then responding was judged stable.

\section{Data Analysis}

The primary data of interest were response rates (responses / min) on the timeout lever. Response rates on the avoidance lever and the percentage of RS shocks avoided were also collected. A discrimination index was calculated for responding on the avoidance lever during time-in and timeout to ensure that the discrimination between time-in and timeout was maintained. Overall shock-frequency reduction was calculated by subtracting the mean rate of shocks in the timeouts from the mean rate of shocks in time-in. Overall response-effort reduction was calculated by subtracting the mean rate of avoidance responding in the timeouts from the mean rate of avoidance responding in time-in.

Unless otherwise stated, when the means are reported, the data are from the last 10 sessions of each condition and the error bars represent one standard deviation. When the medians are reported, the data are from the last 10 sessions of each condition and the error bars represent the interquartile range. 


\section{Experiment 1}

The results of Foreman (2009) suggested that the presence of shocks in timeout can degrade the reinforcing value of timeouts. In that study, the proportion of timeouts with shock was not directly manipulated; rather, the proportion varied as a function of the rat's avoidance behavior in time-in. In some cases, in the initial exposure to the yoking conditions, many timeouts contained shock and timeout responding decreased. The present experiment directly manipulated the frequency of timeouts with shock. This manipulation was intended to permit a more nuanced analysis of shock-frequency reduction than in Foreman (2009), because a wider range of shock frequencies could be obtained. Shocks were delivered in timeout regardless of the rats' avoidance proficiency in time-in. The probability of timeouts with shock was raised across conditions.

\section{Method}

Three rats served as subjects. In the Shock-Free baseline, no shocks were delivered during timeouts. In subsequent conditions, one shock was delivered in some of the timeouts, 1 min after the timeout had begun. Across conditions, the probability of a timeout with shock was raised in increments of .02 until timeout responding decreased to at least $50 \%$ of responding in the Shock-Free baseline. The initial probability (.02) was selected based on the percentage of timeouts with shock in the yoking conditions for each rat in Foreman (2009). Approximately 50 timeouts typically occur per session, so in the $p=.02$ condition, a shock was delivered in about one timeout each session. The specific timeouts with shocks were determined randomly by the computer program during the session. Immediately before a timeout, the computer program would select a random number from 1 to 100 . Then, this number would be compared to the condition criterion. In the $p=.02$ condition, for example, if this number was a 1 or a 2 , then a 
shock would be delivered during the timeout. Responses on the avoidance lever during timeout had no scheduled consequences but were recorded.

The probability of a timeout with shock that produced substantial reductions in timeout responding differed among the rats. The terminal probability was $p=.10$ for Rat AF5 and $p=$ .06 for Rat AF12. For Rat AF6, at $p=.12$, response rates had not decreased but were higher than in the Shock-Free baseline. To decrease timeout response rates more quickly, the probability was raised by.02 every two days until response rates began to decrease. This occurred at $p=.22$ and 29 sessions were conducted to allow responding to stabilize at this terminal probability.

\section{Results}

Avoidance and timeout response rates. Table 1 shows the programmed probabilities and the obtained probabilities of timeouts with shock aggregated over the last 10 sessions of each condition. The number of timeouts that occurred in each session was not fixed but depended on the rats' behavior. The timeouts that included a shock were selected on a probabilistic basis, and the obtained probabilities could deviate from the programmed probabilities. The obtained deviations were small, generally less than .01. Considering all 15 conditions in which the programmed probability was greater than zero, the mean discrepancy was less than .001 .

Avoidance responding was maintained throughout the experiment. Table 2 shows mean rates of responding on the avoidance lever during the last 10 sessions of each condition, during time-in (when the shock-postponement schedule was operative) and timeout. Although response rates during time-in varied somewhat across the conditions, no systematic differences are apparent. Avoidance responding almost never occurred during timeout.

The primary measure of interest was the rate of responding on the timeout lever. Figure 4 shows mean rates of responding on the timeout lever during the last 10 sessions of each 
condition. These rates are also listed in Table 2. For Rat AF5, the general trend was a decrease in timeout response rates across the conditions, although there was little change from $p=.04$ to $p$ $=.08$ conditions. For Rat AF6, the general trend was an increase in rates, although there was little change from the $p=0$ to the $p=.08$ conditions, until the $p=.22$ condition, when rates fell by almost 1 response per min. The mean rate of timeout responding in the $p=.22$ condition did not meet the 50\%-of-baseline-responding criterion, but because of time constraints no further conditions were conducted. For Rat AF12, response rates decreased across the conditions, and there was a large amount of variability in rates within the conditions. Increasing the probability of a timeout with shock did not produce orderly decreases in timeout response rates across conditions for most of the rats.

Shock-frequency reduction. Why did timeout responding remain unchanged or increase when the probability of timeouts with shock was raised? One potential explanation is that despite the occurrence of shocks in some timeouts, the overall frequency of shock was still reduced from time-in to timeout. Because in some cases the time-in shock rates increased when the probability of a timeout with shock was raised, the rate of shock during time-in may have been higher than the rate during timeout. If this were the case, then this shock-frequency reduction could reinforce timeout responding.

The mean shock rates in time-in and timeout are listed in Table 2 . The probability manipulation was intended to decrease the shock-frequency reduction afforded by the timeouts across conditions, because the timeout shock rate would increase and eventually eliminate or make negative the reduction in shocks from time-in to timeout. This was predicated on the assumption that time-in shock rates would be relatively stable across the conditions. Instead, shock rates in time-in varied considerably. In most conditions, shock-frequency reduction 
remained positive, and in some cases increased, as the probability of timeouts with shock was raised. For two of the rats, time-in shock rates were higher than timeout shock rates in all conditions (Rat AF5) or in all but the final condition (Rat AF6). For Rat AF12, time-in shock rates were higher in the first 3 conditions and identical in the final condition. A gradual change in the shock-frequency reduction afforded by the timeouts did not occur. Instead, shock-frequency reduction remained positive in all conditions (Rat AF5), became negative (Rat AF6), or was eliminated (Rat AF12, in only the final condition).

In Figure 5, timeout response rates are plotted against the shock-frequency reduction for each of the last 10 sessions of each condition. If shock-frequency reduction is positive, then more shocks occurred in time-in than in timeout. If shock-frequency reduction is negative, then more shocks occurred in timeout than in time-in. If shock-frequency reduction is zero, then the same number of shocks occurred in time-in and in timeout. For Rat AF5, most of the data points are located around a shock-frequency reduction of zero, even in the conditions in which the probability of timeouts with shock was relatively high and timeout responding was maintained. The range of timeout response rates was from around 1 response per min to 5 responses per min. This variability would not be expected if shock-frequency reduction were responsible for the changes in timeout response rates. The highest - and lowest - rates of timeout responding occurred when there was little to no reduction in shocks from time-in to timeout, rather than when the reduction was substantial. For Rat AF6, there was a reduction in the frequency of shocks from time-in to timeout in most of the stable sessions, as most of the data points are to the right of zero. Even when there was relatively substantial shock-frequency reduction, timeout response rates were low (particularly in the $p=.08$ condition). Like Rat AF5, there was a range of timeout response rates when shock-frequency reduction was positive, from approximately 0.5 
to 3.5 responses per min. The data for Rat AF12 are consistent with the other rats; most of the data points are located around a shock-frequency reduction of zero. When shock-frequency reduction was highest however, timeout responding was maintained at relatively high rates.

Response-effort reduction. Another possibility is that the reduction in response effort from time-in to timeout may have reinforced timeout responding. During time-in, the rat must respond on the avoidance lever to avoid shocks, but this avoidance contingency is absent during timeout. There was a substantial reduction in avoidance responding from time-in to timeout (see Table 2). Inspections of response patterns on the cumulative records indicate that the occasional responses during timeout were likely shock-elicited responses.

Figure 6 shows timeout response rates plotted against response-effort reduction for each of the last 10 sessions in each condition. Response-effort reduction was calculated by subtracting the timeout avoidance response rate from the time-in avoidance response rate for each of the last 10 sessions of each condition. For Rats AF5 and AF6, the data points are clustered between 3 and 7 on the $\mathrm{x}$-axis reflecting the limited range of obtained avoidance response rates. The experiment was not designed to manipulate rates of avoidance responding, and avoidance responding - and therefore response-effort reduction - remained relatively stable across the conditions for all of the rats. Although response-effort reduction was consistent across conditions, timeout response rates varied considerably. For Rat AF12, there was more variability in both response-effort reduction and timeout responding, and when response-effort reduction was the highest, timeout response rates were relatively low. This occurred in the conditions with higher probabilities of shocks in timeout. The shocks during timeout may have degraded the reinforcing efficacy of the timeout, and therefore responding was allocated primarily to the avoidance lever during these conditions. 
Effects of shock. There were no constraints on which timeouts in the probability conditions could include a shock. The first timeout or the last timeout in a session could include a shock, and shocks could occur in consecutive timeouts. The location of the first timeout with shock might have affected rates of timeout responding - a shock delivered in the first timeout of a session could affect subsequent timeout responding more than a shock delivered in a later timeout. Figure 7 shows timeout response rates plotted against the first timeout with shock. In some sessions, no shocks occurred during timeouts. These sessions were included in the figure, and the data points were placed at the $80^{\text {th }}$ timeout position on the $\mathrm{x}$-axis. The sessions consisted of 60 min of time-in, so given the VI 45-s schedule on the timeout lever, as many as 80 timeouts could occur in a session (3,600 s of time-in divided by a mean time between timeouts of $45 \mathrm{~s})$. For all of the rats, there was essentially no relation between timeout response rates and the first timeouts with shock. A range of timeout response rates was obtained, and even when a shock occurred in a timeout early in the session, timeout response rates were often relatively high. These data indicate that timeout response rates did not vary as a function of the first timeout with shock.

Although the location of timeouts with shock did not affect timeout responding over the course of the session, shocks during timeout may have disrupted patterns of responding within a session. Examining the interresponse times (IRTs) on both levers after timeouts with and without shock may reveal changes in behavior that cannot be detected by examining the timeout response rates alone. The IRTs come from the periods of time-in between timeouts. Figure 8 shows the median IRTs on the timeout lever (left column) and avoidance lever (right column) following timeouts with and without shock in each condition. For all of the rats, the median IRTs following timeouts with shock were longer than the IRTs following timeouts without shock in all 
conditions. There was a large amount of variability in the IRTs following timeouts with shock as indicated by the size of the interquartile ranges (error bars in Figure 8). For Rats AF6 and AF12, the magnitude of the differences decrease by the last condition, while for Rat AF5 the magnitude remains large. If timeouts with shock lead to a general suppression in responding, then similar patterns of IRTs would be obtained on the avoidance lever. There were no such differences on the avoidance lever, however, indicating that the effects of timeouts with shock on responding were limited to responses on the timeout lever.

\section{Discussion}

The probability manipulation was successful in increasing the frequency of timeouts with shock across the conditions. For two of the rats, Rats AF5 and AF12, reductions in timeout responding occurred in the first condition with shocks in timeout, the $p=.02$ condition. For the other rat, Rat AF6, timeout responding was maintained near Shock-Free baseline levels in the conditions with the relatively lower probabilities of shock $(p=.02$ to $p=.08)$. More drastic reductions in timeout responding occurred at higher probabilities: $p=.10$ for Rat AF5, $p=.22$ for Rat AF6, and $p=.06$ for Rat AF12.

The manipulation was intended to produce a graded change in shock-frequency reduction across conditions; shock-frequency reduction would decrease as the probability of timeouts with shock was raised. This did not occur, however, because the rate of shock in time-in increased across conditions for two of the rats, and shock-frequency reduction remained positive across these conditions. The shocks in timeout may have disrupted avoidance behavior during time-in, which led to increased shock rates in time-in. For the shock-frequency reductions that were obtained, there was not a strong relation between changes in timeout responding in changes in shock-frequency reduction. 
For all of the rats, changes in timeout responding were not correlated with changes in response-effort reduction. Rates of avoidance responding were not manipulated, and rates did not change substantially from condition to condition, even when avoidance proficiency declined.

Even at probabilities of shock in timeout that did not produce changes in overall rates of timeout responding, the pattern of responding after a timeout with shock was altered. This was observed in changes in the length of the IRTs after timeouts with shock. IRTs were systematically longer following timeouts with shock compared to timeouts without shock. This change in response patterning indicates that shocks in timeout temporarily decreased the reinforcing efficacy of the timeouts, but this effect was obscured in the overall response rates. While the shocks in timeout were not sufficiently aversive to decrease overall rates of responding in some cases, they did produce brief decrements in the reinforcing value of timeout.

\section{Experiment 2}

In Foreman's (2009) Local-Yoking condition, when the temporal sequence of shocks that occurred in 2-min of time-in were played back in the subsequent timeout, timeout responding was maintained for three of the four rats. In contrast, in Molar Yoking, when the sequence of shocks in time-in from the previous session was played back in the timeouts, timeout responding was reduced for three of the four rats. It is possible that the temporal sequence of shocks that occurred in the 2 min of time-in in Local Yoking may have served a discriminative or signaling function for the shocks that would be delivered in timeout. This potential discriminative function was absent in Molar Yoking because the sequence of shock in the timeouts was obtained from the previous session. To assess whether the shocks in Local Yoking in Foreman played a discriminative role, the local method of eliminating shock-frequency reduction was preserved, but the shocks were no longer played back in the same temporal sequence. In a new condition, 
Random Local Yoking, the temporal sequence of shocks that occurred in the 2 min of time-in preceding a timeout was randomized and played in the subsequent timeout. This manipulation eliminated the potential signaling function of the specific temporal locations of shock in time-in in Local Yoking.

\section{Method}

Four rats served. The experimental conditions were defined in terms of the nature of the timeout. As in Experiment 1, in the Shock-Free baseline, the timeouts were conventional in that no shocks were scheduled. In the other two conditions, shocks were delivered during timeout so that the rate of received shock during timeout was yoked to the rate during time-in. The conditions differed in terms of the temporal location of the shocks delivered in timeout.

In the Local-Yoking condition, the sequence of shocks during any 2-min timeout was the same as the sequence during the $2 \mathrm{~min}$ of time-in that immediately preceded the timeout. The computer program recorded the time at which each shock occurred during time-in. When a timeout was presented, the program replayed the most recent 2 min of the resulting event record. The goal was to ensure that no short-term change occurred in the shock rate from time-in to timeout.

In the Random-Local-Yoking condition, the number of shocks during any 2-min timeout was the same as the number during the 2 min of time-in that immediately preceded the timeout, but the temporal locations of the shocks in the timeout were randomized. For example, if a shock was delivered $5 \mathrm{~s}$ into the 2-min segment of time-in preceding the timeout, the computer program would select a number from 1 to 120 at random. If the computer program selected the number 31, for example, then the shock would be delivered $31 \mathrm{~s}$ into the timeout. If more than one shock occurred in the 2-min preceding the timeout then the program would select a new random 
number for each shock that occurred. There was no change in the local rate of shock from timein to timeout, but the specific temporal location of the shocks was randomized from time-in to timeout.

For two rats the conditions were presented in this order: Shock Free, Local Yoking, Shock Free, Random Local Yoking, Shock Free, Random Local Yoking, Shock Free, Local Yoking. For the other two rats, the order of the two initial yoking conditions was reversed.

\section{Results}

Avoidance and timeout response rates. Avoidance responding was maintained throughout the experiment. Table 3 shows mean rates of responding on the avoidance lever during time-in, when the shock-postponement schedule was operative, and timeout. The table also shows the consequences of avoidance responding in the form of received shocks rates and the percentage of RS shocks avoided. Although response rates during time-in varied somewhat across the conditions, no systematic differences are apparent. For all of the rats in most conditions, avoidance proficiency was well above $80 \%$ and rates of received shocks were low. Avoidance responses rarely occurred during timeout.

Mean response rates on the timeout lever are shown in Figure 9 and Table 3. Timeout responding was maintained in all conditions. Small, unsystematic changes in rates among the conditions were obtained for three of the four rats. Systematic changes in response rates between the two types of yoking were present for only one rat, Rat AF4. Timeout response rates were higher than the previous baselines in the Random-Local-Yoking conditions and lower than the previous baselines in the Local-Yoking conditions. These differences in response rates were not dramatic, and they were in the opposite direction of what would be expected if the temporal position of shocks in Local Yoking served a discriminative function. If this were the case, Local 
Yoking response rates would be equal or higher than the preceding baseline, not lower. Instead, for Rat AF4, response rates in Local Yoking were lower than the previous baselines and response rates in Random Local Yoking were higher than the previous baselines.

Shock-frequency reduction. Although local shock-frequency reduction was eliminated in the yoking conditions, it is possible that the rats' behavior was sensitive to overall reductions in shock frequency afforded by the timeouts. Figure 10 shows each session's timeout response rate plotted against the shock-frequency reduction for that session. In most of the sessions for Rats AF2 and AF7, shock-frequency reduction was close to zero. This reflects the relatively high avoidance proficiency in most conditions - shock-frequency reduction was near zero because shocks were a rare occurrence in time-in and timeout. Despite the low shock rates and minimal shock-frequency reduction, a range of timeout response rates was obtained. When shockfrequency reduction was close to zero, Rat AF2's timeout response rates ranged from approximately 1.5 to 4 responses per min, and Rat AF7's rates ranged from approximately 1 to 4 responses per min. For Rat AF4, shock-frequency reduction tended to be higher in the ShockFree baselines than in the yoking conditions. Although shock-frequency reduction was higher in many of the Shock-Free sessions (indicated by the open triangular and circular data points located to the right of zero on the $\mathrm{x}$-axis), timeout responding was lower in these sessions than in the yoking conditions when shock-frequency reduction was close to zero.

Response-effort reduction. In Figure 11, timeout responding is plotted against responseeffort reduction in each session. For all of the rats, the data points are clustered around a narrow range of response-effort reduction and timeout response rates, and this clustering reflects the lack of variability in avoidance and timeout response rates across conditions. Timeout response rates did not vary as a function of response-effort reduction. 
Effects of shock. Figure 12 shows the median IRTs after timeouts with and without shock for the timeout lever (left column) and avoidance lever (right column). The IRTs are from the periods of time-in between timeouts. The median IRTs for each condition were examined and there were no systematic differences among the conditions, so the IRTs following both types of timeouts in all conditions were combined. For three of the rats (Rats AF2, AF7, and AF10), the IRTs on the timeout lever were substantially longer following timeouts with shock. This difference does not reflect a general suppression of responding on both levers, because such differences were not obtained on the avoidance lever. The effect was limited to responses on the timeout lever. Though the effects of the shocks in timeout were not of a frequency to alter rates of timeout responding, they did temporarily affect subsequent responding on the timeout lever. Shocks were a rare occurrence in timeout, and there were many more timeout responses following timeouts without shock than timeouts with shock. For example, for AF10 in the Local Yoking conditions, in a session there were an average of 56 IRTs following timeouts without shock and an average of 7 IRTs following timeouts with shock. To obtain the session response rate, the responses that make up the IRTs following timeouts with and without shock were combined. Because of the low relative frequency of timeouts with shock, the consistent, suppressive effect of shocked timeouts on timeout responding was obscured in the overall rate.

In addition to the local effects of shock on timeout IRTs, there is evidence to suggest that behavior was sensitive to the temporal relation between shocks in time-in and timeout with both types of yoking. With the method of yoking used in both types of conditions (Local Yoking and Random Local Yoking), shocks that are delivered in the 2-min of time-in immediately preceding a timeout were played back in the subsequent timeout. If a response on the timeout lever was delayed by at least 2-min after a shock is delivered in time-in, then no shocks were delivered in 
the subsequent timeout. Figure 13 shows the median post-shock response latencies on the timeout (left column) and avoidance (right column) levers in all of the conditions. The data from the last 10 sessions of each of the replications of the conditions were pooled and the medians were obtained from the pooled data. Though there is a large amount of variability (as indicated by the size of the interquartile ranges) for all rats, the median latency after receiving a shock in time-in is longer in the yoking conditions than in the baseline conditions. One potential explanation is that after a shock is delivered in time-in, the rat spends more time pressing the avoidance lever than the timeout lever, regardless of whether the shock could be delivered in the next timeout. If this were the case, however, then the latency would be long in all conditions, not just in the yoking conditions. The latencies were longer in the yoking than in the baseline conditions, indicating that behavior was sensitive to the method of yoking shocks in both types of yoking conditions.

\section{Discussion}

There were no systematic differences in timeout responding between the two types of yoking for most of the rats. Timeout responding in Local Yoking and Random Local Yoking was maintained at the same rates as in the preceding Shock-Free baselines. When systematic differences were obtained for one rat, Rat AF4, responding in Random Local Yoking was slightly higher than the preceding baselines, and responding in Local Yoking was slightly lower than the preceding baselines. Though these differences were systematic, they were very small. In general, timeouts were just as reinforcing when the temporal position of the shocks in timeout was randomized than when the specific temporal locations of shocks in the timeouts were preserved. These results indicate that the signaling function of shocks in Local Yoking was not the specific temporal locations of the shocks. 
Although the timeout response rates indicate that behavior was not sensitive to changes in the temporal position of the shocks within the timeouts, there is evidence to suggest that behavior was sensitive to the method of yoking the shocks from the 2 -min of time-in to the subsequent timeout that occurred in both Local Yoking and Random Local Yoking. The evidence for this comes from the latencies to respond on the timeout lever after a shock in timein. The latencies were longer in the two types of yoking conditions than in the Shock-Free baseline conditions. These results suggest that the maintenance of responding in Local Yoking in Foreman (2009) may be due to this signaling function of the shocks in time-in in this condition. Further evidence for this sensitivity comes from the data from Foreman (2009) in which two types of yoking were arranged, Local Yoking and Molar Yoking. Unlike the LocalYoking condition, in Molar Yoking, behavior during time-in in a session did not affect the frequency of shocks in timeout in the same session - the shocks in timeout came from the previous day's session. Figure 14 shows the median latencies to respond on the timeout lever after a shock in time-in. For three of the four rats (Rats AF15, AF19, and AF20), there were longer latencies in Local Yoking than in the Shock-Free Baseline and Molar Yoking. The latencies were longer only when the shocks in time-in could occur in the following timeout within the same session.

As in Experiment 1, there was little to no relation between timeout responding and shockfrequency reduction. Shock-frequency reduction was close to zero in most of the conditions for most of the rats, yet timeout response rates varied considerably. Similar results were obtained for response-effort reduction - there was no relation between timeout responding and responseeffort reduction. Also consistent with Experiment 1, the IRTs were longer after timeouts with shock, despite the absence of changes in timeout response rates. The shocks in timeout did affect 
subsequent timeout responding, but given the lower frequency of timeouts with shock, this effect of shock was obscured in the overall rates of responding.

\section{Experiment 3}

In Experiment 1, the reinforcing value of the timeouts was degraded by raising the probability of timeouts with shock across conditions. The shocks that occurred in timeout in Experiment 1 were unsignaled - they were not preceded by a correlated stimulus. Given the research demonstrating that signaled shocks are less aversive than unsignaled shocks, it is possible that the reinforcing value of the timeouts could be restored if the shocks in timeout were changed from unsignaled to signaled. In Experiment 3, a signal (5-s tone) was added before the shocks that were delivered in timeouts after timeout responding had been degraded in Experiment 1 to investigate whether the signals would restore the reinforcing efficacy of the timeouts. If the signals in timeout increase the reinforcing efficacy of the timeouts, then this would suggest that response rates were lower in Molar Yoking in Foreman (2009) because of the absence of signals for shocks in timeout.

\section{Method}

The three rats from Experiment 1 served as subjects. The last condition of Experiment 1 served as the baseline condition, the Unsignaled-Shock condition. In the next condition, the Signaled-Shock condition, a 1000-Hz tone was presented for $5 \mathrm{~s}$ before each shock that was delivered in timeout. Shocks during time-in were still unsignaled. The only change was the addition of the signal before shocks in timeout, the probability of timeouts with shock remained the same as in the Unsignaled-Shock condition. When responding was judged stable, the Unsignaled-Shock condition was repeated. 
The sequence of conditions and the number of sessions in each condition are shown in Table 4. The condition in Experiment 1 in which responding was reduced by $50 \%$ differed across rats. The terminal probabilities from Experiment 1 were $p=.10$ for Rat AF5, $p=.22$ for Rat AF6, and $p=.06$ for Rat AF12.

\section{Results}

Mean avoidance response rates are shown in Table 4. Avoidance responding was maintained in all conditions and there were no systematic differences in avoidance responding among the conditions.

Mean timeout response rates are shown in Figure 15 and Table 4. For comparison, the first bar in the figure represents the timeout response rate from the first condition (Shock-Free baseline) of Experiment 1. The first Unsignaled-Shock bar is the same data from the last condition of Experiment 1. Timeout responding was maintained in all conditions, but the rates of timeout responding were higher in the Signaled-Shock conditions than in the Unsignaled-Shock conditions for all rats. For Rat AF5, there was only a small decrease in timeout responding in the second Unsignaled-Shock condition, so the Signaled-Shock condition was repeated. In the third Unsignaled-Shock condition, timeout responding decreased substantially. For Rats AF6 and AF12, only one Signaled-Shock condition was conducted because timeout responding in the second Unsignaled-Shock condition was similar to responding in the first Unsignaled-Shock condition. For Rats AF6 and AF12, responding in the Signaled-Shock condition was higher than responding in the first condition (Shock-Free baseline) of Experiment 1.

Shock-frequency reduction. In Figure 16, timeout responses per min are plotted against shock-frequency reduction for the last 10 sessions of each condition. For all of the rats, the data points from the Signaled-Shock conditions are located within the same range of shock-frequency 
reduction as the data points from the Unsignaled-Shock conditions, yet timeout responding in the Signaled-Shock conditions was generally higher than in the Unsignaled-Shock conditions.

Response-effort reduction. Figure 17 shows timeout responding plotted against response-effort reduction for the last 10 sessions of each condition. For all of the rats, there was a limited range of response-effort reduction, particularly for Rats AF5 and AF6, as the data points are clustered around 4 on the $\mathrm{x}$-axis. There were no systematic differences in response-effort reduction between the two types of shock; timeout response rates were generally higher in the Signaled-Shock conditions, but response-effort reduction was not differentially larger or smaller in these conditions.

Effects of shock. Figure 18 shows the median IRTs after timeouts with and without shock for each condition for the timeout lever (left column) and avoidance lever (right column). The IRTs are from the last 10 sessions of each condition, and the medians were calculated from the IRTs from each of the last 10 sessions of each replication. For both the Signaled- and Unsignaled-Shock conditions, on the timeout lever, median IRTs were longer following timeouts with shock than following timeouts without shock. Unlike the results of the other experiments, the differences between the lengths of the IRTs were small, and the interquartile ranges overlap in both conditions for all of the rats.

\section{Discussion}

Timeout response rates were higher in the Signaled-Shock conditions than in the Unsignaled-Shock conditions for all of the rats. The higher rates were not due to differences in shock-frequency reduction or response-effort reduction. Adding signals to the shocks restored the reinforcing efficacy of the timeouts that had been degraded by the addition of an increasing probability of shocks in timeout in Experiment 1. 
These results are consistent with the extensive literature demonstrating that rats prefer signaled to unsignaled shock (Badia \& Culbertson, 1972), and that less response suppression occurs following shocks that are signaled rather than unsignaled (Hymowitz, 1973, 1976, 1977). The shocks in timeout that were preceded by signals were less aversive than the shocks that were not. For two of the rats, response rates in the Signaled-Shock conditions returned to Shock-Free baseline levels, even though the programmed probability of timeouts with shock in these conditions had reduced responding to at least $50 \%$ of baseline in the preceding condition.

Why did the signals restore the reinforcing efficacy of the timeouts? In the typical timeout-from-avoidance procedure, the timeouts are a signaled shock-free period, or a safety period - no shocks are delivered during the timeouts. Adding shocks to the timeouts on a probabilistic basis denigrated this safety period - sometimes timeouts free of shock and sometimes they were not. Adding signals before the shocks in timeout, as in Experiment 3, reinstated the timeout as a signaled period of safety. When the signal was not on in timeout, no shocks were ever delivered. Previous research comparing signaled and unsignaled shocks has provided evidence for this safety-signal hypothesis. Seligman, Maier, and Solomon (1971) were the first to fully explicate this hypothesis in relation to signaled and unsignaled aversive events, and a series of experiments by Badia, Culbertson, and Lewis (1971) and Badia and Culbertson (1972) tested the hypothesis.

Badia et al. (1971) used a free-operant procedure to assess preference for signaled or unsignaled shock. Responses on one lever avoided shocks and responses on another lever (the changeover lever) changed the context from one in which light was off above one of the levers and shocks were unsignaled to one in which light was on above one of the levers and shocks were signaled (the procedure is described in more detail in the Introduction of this document). 
Extinction of the changeover response was evaluated, and three different types of extinction conditions were conducted. In the first extinction condition, responses on the changeover lever now produced no stimulus changes - the rat remained in the unsignaled condition regardless. In the other two extinction conditions, responses on the changeover lever produced different stimulus changes. In the second extinction condition, responses on the changeover lever only turned on the light above one of the levers for $60 \mathrm{~s}$ - the light correlated with the signaled context - but no signals were presented before shocks. In the third extinction condition, responses on the changeover lever only produced signals before shocks for $60 \mathrm{~s}-$ the light above the lever was not turned on. If the rat responded with sufficient frequency in this third extinction condition, all of the shocks were signaled but the lever light that was correlated with the signaled condition was never on. For 6 of the 8 rats, changeover responding showed the greatest resistance to extinction in the second extinction condition, in which responses on the changeover lever only produced stimuli correlated with the signaled context. Comparable decrements in responding occurred in the first and third extinction conditions. These results suggest that preference for signaled shock is controlled by the presentation of stimuli that signal a period of safety.

Badia, Harsh, Coker, and Abbott (1976) conducted a more extensive test of the safety hypothesis. A similar procedure was used in Badia et al. (1971); responses on one lever avoided shocks and responses on another lever changed the context from unsignaled to signaled shock. In Experiment 1, in the signaled context, shocks were always preceded by signals, but the probability of a shock following a signal was manipulated. In some cases, no shock followed the signal and in some cases a shock followed the signal. Changeover responses were unaffected by these changes in probability. In Experiment 2, in the signaled context, the probability of a shock 
in the presence of a signal remained constant, but the probability of a shock in its absence varied. Sometimes a shock was preceded by a signal and sometimes it was not. Changeover responding decreased as the dependability of a signal given a shock was decreased. These results lend additional support to the safety hypothesis, because changeover responding decreased only when the dependability of the stimulus identifying the shock-free period was degraded.

The present results can be interpreted in terms of the safety hypothesis. Adding signals before the shocks in timeout reestablished timeouts as a safety period. When the signal was not on, no shocks were ever delivered.

\section{General Discussion}

The purpose of the present set of experiments was to investigate the factors that may have contributed to the discrepant results obtained in Foreman (2009). In that study, to assess the effects of shock-frequency reduction on the reinforcing efficacy of timeout, shock-frequency reduction was equated from time-in to timeout via two different methods. In one method, Molar Yoking, the sequence of shocks in time-in in the previous session were played back during the timeouts, and in the other method, Local Yoking, the shocks in the 2-min of time-in preceding a timeout were played back in the timeout. Discrepant results were obtained between the two types of yoking: in general, timeout responding decreased in Molar Yoking and increased or stayed the same in Local Yoking. In the present study, several manipulations were carried out to shed light on the variables that may have contributed to these differences.

In Foreman (2009), the lower rates of timeout responding in Molar Yoking may have been due to the sudden increase in shocks from time-in to timeout in the first few sessions of the condition. The rapid increase in shock frequency from time-in to timeout led to rapid decreases in timeout responding. In Experiment 1 of the present study, the frequency of timeouts with 
shock was directly manipulated to assess the point at which the reinforcing efficacy of timeout would be sufficiently degraded to reduce timeout responding. The probability of a timeout with shock was raised across conditions. The manipulation was successful at degrading the reinforcing value of the timeouts, and timeout response rates decreased to at least $50 \%$ of the Shock-Free baseline rates for two of the three rats. For the third rat, timeout responding was reduced to only $78 \%$ of baseline before the experiment was ended due to time constraints. When the probability of a timeout with shock was relatively low in the initial conditions of the experiment, the reductions in timeout responding were not as dramatic as in the Molar Yoking conditions in Foreman (2009). For one rat, responding in these early conditions was maintained at Shock-Free baseline rates. These results in these early conditions of Experiment 1 suggest that the decreases in timeout responding in Molar Yoking in Foreman were likely due, at least in part, to the sudden increase in shocks in timeout at the beginning of the condition.

Another potential reason for the differences in timeout responding between Molar and Local Yoking in Foreman (2009) was that the method employed to yoke the time-in shocks in Local Yoking may have served a signaling function for the shocks delivered in timeout. In Local Yoking, the temporal sequence of shocks delivered in the previous 2-min of time-in were played back in the subsequent timeout. If the shocks in timeout in Local Yoking were signaled, then timeout responding may have been higher because the shocks in timeout were less aversive than the shocks in timeout in Molar Yoking. Experiment 2 investigated whether the temporal location of shocks or just the mere occurrences of shock in time-in in Local Yoking served a signaling function for the shocks delivered in timeout. Two yoking conditions were arranged: Local Yoking and Random Local Yoking. The Local Yoking condition was identical to the condition in Foreman. In Random Local Yoking, the shocks to be delivered in timeout were played back in 
random temporal locations in the timeout. There were no systematic differences in timeout responding between the two yoking conditions for most of the rats, and timeout responding was maintained with both types of yoking. These results indicate that the specific temporal locations of the shocks in time-in did not serve a signaling function for the shocks in timeout.

In the Molar Yoking condition in Foreman (2009), the shocks were yoked from the previous session; therefore, the occurrence of shocks in time-in could not serve as signals for the shocks in timeout. The absence of signals for shocks in timeout may have contributed to the decreased timeout response rates obtained in the Molar Yoking condition. Experiment 3 assessed whether adding explicit signals before shocks in timeout could restore the reinforcing efficacy of the timeouts after timeout responding had been degraded by shock in Experiment 1 . The Unsignaled-Shock conditions (and the last condition of Experiment 1) were similar to Molar Yoking - the frequency of shocks in timeout was unrelated to the rat's behavior within the session. In the Signaled-Shock condition, the shocks in timeout were still delivered on a probabilistic basis, but a 5-s tone was added before each shock. Adding signals before the shocks restored the reinforcing efficacy of the timeouts. Timeout responding increased as a function of the signaling manipulation. These results suggest that timeout responding may have been lower in Molar Yoking compared to Local Yoking because of the absence of signals preceding the shocks in timeout.

In the following subsections, the results and implications of the primary variables of interest - shock-frequency reduction, response-effort reduction, and signals - will be discussed. Additionally, applications of the timeout procedure and research with signaled and unsignaled aversive events to anxiety and phobic disorders in humans and other populations will be described. 


\section{Shock-Frequency Reduction}

The present experiments attempted to manipulate shock-frequency reduction with two methods. The first method was in Experiment 1, in which to obtain a range of shock-frequency reduction from time-in to timeout, the probability of a timeout with shock was raised across the conditions. This was predicated on the assumption that avoidance proficiency would be stable from condition to condition. Instead, for two of the rats, avoidance proficiency decreased as the probability of shock in timeout was raised and more shocks were delivered in time-in. This prevented a gradual change from positive shock-frequency reduction to negative shockfrequency reduction across conditions. Instead, shock-frequency reduction was close to zero in most of the conditions. In the conditions in which shock-frequency reduction did deviate from zero, in general, shock-frequency reduction was positive.

The second method to manipulate shock-frequency reduction was in Experiment 2, in which local shock-frequency reduction was eliminated by playing back the shocks from the preceding 2-min of time-in in the subsequent timeout. This manipulation was successful, and just as in Foreman (2009), responding in the yoking conditions was maintained or increased from the previous Shock-Free baselines, in which no shocks were delivered in the timeouts. The absence of local shock-frequency reduction did not degrade the reinforcing efficacy of the timeouts.

Despite successfully eliminating local shock-frequency reduction in Experiment 2, the present experiments did not provide an adequate test of shock-frequency reduction as a reinforcer for timeout responding. A limited range of shock-frequency reduction was obtained in all of the experiments. To satisfactorily assess the relation between shock-frequency reduction and timeout responding, a wider range of shock-frequency reductions would need to be obtained. Instead, in the present experiments, shock-frequency reduction was generally close to zero. When there was a wider range of values, they were mostly positive with a limited range of 
negative values. It may be difficult to assess a sufficient range of shock-frequency reduction using the timeout-from-avoidance procedure.

Shock-frequency reduction may play an important role in reinforcing timeout responding, but it is difficult to draw any conclusions from the present data because only a narrow range of shock-frequency reduction was obtained. Other studies have shed more light on the role of shock-frequency reduction. For example, in Perone and Galizio’s (1987) study, timeout responding was maintained despite low frequencies (generally 0.3 shocks per min or lower) of shock in time-in. In Courtney and Perone (1992), shock frequency was manipulated by changing the parameters of the shock deletion schedule between the components of a multiple schedule across conditions, and timeout responding was relatively insensitive to changes in shock frequency. Although the evidence from these studies suggests that shock-frequency reduction may not be the factor in the reinforcing efficacy of timeout, it still may play a role. Although the above studies found weak relations between shock-frequency reduction and timeout responding, shocks never occurred in the timeouts, and shock-frequency reduction was always positive. It may be that the reinforcing value of the timeout does not increase much with increases in shockfrequency reduction above 0 (if shock-frequency reduction is 0 , then there is no change in shock frequency from time-in to timeout). It may be the case, however, that negative shock-frequency reduction has a substantial effect on the reinforcing value of the timeout. Evidence for this comes from Foreman (2009), in which increases in shock from time-in to timeout in the Molar Yoking conditions decreased rates of timeout responding for most of the rats. Additionally, in Experiment 1 of the present study, when shock-frequency reduction was negative, timeout responding was relatively low (Figure 5).

\section{Response-Effort Reduction}


The present experiments were not designed to manipulate response-effort reduction. Avoidance response rates were stable across the conditions for all of the rats, and therefore response-effort reduction remained relatively stable across the conditions. Similar to the shockfrequency reduction data, only a limited range of response-effort reduction was obtained. Therefore, it is difficult to draw any conclusions about the role of response-effort reduction in reinforcing timeout responding with the data from the present experiments.

It is plausible that response-effort reduction plays an important role in reinforcing timeout responding. As Perone and Crawford (1999) pointed out, avoidance responses typically occur at a high rate in timeout studies, therefore a reduction in their frequency from time-in timeout may be more salient than other events that change in frequency from time-in to timeout (e.g., shocks). In the present study, response-effort reduction from time-in to timeout was a consistent factor in all of the experiments. Indeed, unlike shock-frequency reduction, response-effort reduction is always present in the standard timeout procedure - there is always a reduction in responding from time-in to timeout. Courtney and Perone (1992) have conducted the most thorough analysis of the roles of shock frequency and response-effort reduction in reinforcing timeout responding. Using the generalized matching law to analyze the results of their study, and they found that timeout responding was sensitive to changes in avoidance responding but was insensitive to changes in shock frequency.

Unlike shock-frequency reduction, there may be a more graded relation between response-effort reduction and the reinforcing value of the timeout across a wider range of values - increases in response-effort reduction produce corresponding increases in the value of the timeouts. In contrast, shock-frequency reduction may only affect the reinforcing value of the timeouts when it is negative, and increases in shock-frequency reduction in zero may not have a 
graded effect on timeout responding. If the value of the timeout is not affected dramatically by changes in positive shock-frequency reduction, yet the value is affected by response-effort reduction across a wider range, then the results of Courtney and Perone and Perone and Galizio make sense.

\section{Signals}

The role of signals in controlling behavior was studied in two different ways in the present experiments. In Local Yoking in Foreman (2009), the temporal locations of the shocks in time-in may have served a signaling function for the shocks delivered in timeout, because they were played back in the same temporal arrangement. In Experiment 2, the potential signaling function of the locations of shocks in time-in was investigated by exposing the rats to Local Yoking, in which the shocks in timeout occurred in the same temporal locations as in time-in, and Random Local Yoking, in which the location of shocks was randomized from time-in to timeout. The results of Experiment 2 indicate that the specific temporal locations of shocks in Local Yoking did not serve a signaling function because there were no clear systematic differences in timeout responding between the two types of yoking for most of the rats.

Although the specific temporal locations of shocks in timeout did not serve a signaling function, there is evidence to suggest that the shocks in time-in in both types of yoking in Experiment 2 did function as signals. In the yoking conditions (both Local and Random Local), a shock delivered in the 2 min of time-in preceding a timeout was delivered in the subsequent timeout. Sensitivity of behavior to this manipulation was evident in the latencies to respond on the timeout lever after a shock was delivered in time-in. Latencies to respond on the timeout lever were longer in the yoking conditions. If a shock was delivered in time-in, then there was a longer period of time to the next timeout response than in the baseline conditions. If a timeout 
response was delayed by at least 2 min after a shock, then that shock would not be played back in timeout.

The second way signals were investigated in the present experiments was in Experiment 3. The first condition of this experiment, the Unsignaled-Shock condition was also the last condition of Experiment 1, in which the reinforcing efficacy of timeout had been degraded by adding shocks to the timeouts. A signal, a brief tone, was added before each shock in timeout in the Signaled-Shock condition. Timeout responding increased in the Signaled-Shock condition and decreased when the signals were removed in the subsequent Unsignaled-Shock condition. These results are consistent with the extensive literature on the relative aversiveness of signaled and unsignaled shocks; previous research has demonstrated that rats prefer signaled to unsignaled shocks (Badia \& Culbertson, 1972; Lockard, 1963), and in punishment procedures, less response suppression occurs when shocks are signaled rather than unsignaled (Hymowitz, 1973, 1976, 1977). In the present experiment, signaled shocks in timeout were less aversive than unsignaled shocks, as response rates were higher in the Signaled-Shock conditions. By adding signals to the shocks in timeout, the reinforcing value of the timeouts was restored. These results suggest that timeout responding in Molar Yoking in Foreman (2009) may have been reduced, in part, because of an absence of signals for the shocks in timeout.

There have been several hypotheses proposed for why signaled shocks are less aversive than unsignaled shocks, and the results of the present experiment can be interpreted according to the hypothesis that has received the most support, the safety signal hypothesis (Badia, Harsh, \& Abbott, 1979). According to this hypothesis, the signals delineate two orthogonal periods of time: a safety period and a shock period. The shock periods are typically brief, and the signals identify a long period of safety from shock - when the signal is not on, no shocks will be 
delivered. In the Shock-Free baselines in the present experiments, the timeouts were a signaled period of safety from shocks. Delivering shocks on a probabilistic basis in the timeouts degraded the timeout as a safety period. A similar manipulation was carried out by Badia, Harsh, Coker, and Abbott (1976). They used a free-operant procedure in which, in the baseline condition, rats responded to switch the context from one in which response-independent shocks were always unsignaled to one in which shocks were always signaled (as in Badia \& Culbertson [1972], described in detail in the introduction). In the second experiment, shocks were delivered without a preceding signal on a probabilistic basis in one context, and shocks were always unsignaled in the other context. The probability of a signal before a shock delivery was manipulated across conditions. This manipulation degraded the safety period - shocks could now occur in the absence of a preceding signal. Rats spent a decreasing amount of time in the signaled condition as the probability of a shock without a signal was raised. When the dependability between shocks and preceding signals was restored, rats spend the majority of the session in the signaled context.

In Experiment 1 of the present study, the integrity of the signaled safety period, the timeout, was degraded. The timeouts were no longer perfectly correlated with safetysometimes shocks occurred and sometimes they did not. With a sufficient frequency of shocks in timeout, the reinforcing value of the timeout was reduced and timeout responding decreased. In Experiment 3, the integrity of the safety periods was restored by adding a tone before the shocks in timeout. The absence of the tone in timeout was now a safety signal, because a shock was never delivered in timeout when the tone was not playing.

The results of Badia et al. (1976) suggest that safety is not an absolute. The rats did not immediately stop spending time in the signaled context when some of the shocks were not preceded by signals and the safety period was degraded. These results are similar to the results of 
the final conditions of Experiment 1 and the first condition of Experiment 3 in the present study. As the probability of shock in timeout was raised, timeout responding did not decrease rapidly for most rats, even though the role of timeout as a safety period was degraded. The rats did not stop responding on the timeout lever immediately after receiving a shock in timeout.

One potential issue with the present study is that when the session ended, the rats were essentially in a timeout. The stimulus conditions were identical to the timeouts that occurred within a session: the houselight and white noise were turned off and no shocks were delivered. The rats were removed from the operant chambers when all of the rats' sessions were finished. Therefore, the rat may have remained in the chamber for as little as a minute to over an hour. This could have increased the reinforcing efficacy of timeout as a period of safety because no shocks were ever delivered during this extended timeout. There is evidence to suggest that this may not necessarily have been the case, however. In an unpublished study by Baron, Williams, and Posner described by Perone (2003), rats responded on a progressive ratio (PR) schedule of signaled timeout from avoidance. In PR schedules, the response requirement increases after each reinforcer over the course of the session. The effectiveness of the reinforcer is evaluated by the terminal ratio, which is the highest ratio the animal will complete before responding ceases. In Baron et al.'s study, the duration, or magnitude, of the timeouts was increased across conditions, from $1 \mathrm{~min}$ to $8 \mathrm{~min}$. As the duration of the timeout was raised, the mean ratio increased. If the extended response-independent timeout that occurred at the end of the session was controlling behavior, then it is unlikely that a graded effect of magnitude would be obtained. In future studies, one way to reduce this problem of the extended post-session timeout is to alter the stimulus changes in timeout so that they are not identical to the stimulus changes at the end of the session. 


\section{The Significance of Signaled vs. Unsignaled Aversive Events}

The finding that signals before the shocks in timeout decreased the aversiveness of those shocks is applicable to laboratory research on factors affecting anxiety disorders in humans. In the broader literature, signaled and unsignaled events are typically characterized as "predictable" and "unpredictable," respectively. Unpredictability of aversive events is considered to be a key factor in the development and maintenance of anxiety disorders (Chorpita \& Barlow, 1998; Foa, Zinbarg, \& Rothbaum, 1992; Mineka \& Zinbarg, 1996), and laboratory research with humans has been conducted to assess preference for predictable or unpredictable aversive events. Lejuez, Eifert, Zvolensky, and Richards (2000) conducted a laboratory study examining preferences for signaled or unsignaled 20-s administrations of $20 \%$ carbon dioxide $\left(\mathrm{CO}_{2}\right)$ enriched air delivered through a mask. This stimulus was selected because of its ability to induce panic in human subjects and mimic the symptomology associated with a panic attack (faintness, shortness of breath, etc.). At the beginning of the experiment, the subjects were assessed and rated as high- or low-anxiety individuals. In Phase 1, the masked subjects were seated in front of a computer. If a "T" was presented on the screen, the subsequent trial was predictable. In this 1-min trial, a 200$\mathrm{Hz}$ tone was played during the part of the trial in which $\mathrm{CO}_{2}$-enriched air was not delivered, and

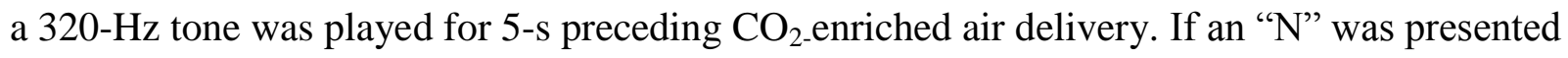
on the screen, the trial was unpredictable. In the subsequent 1-min trial, no tones were played. Some trials included $\mathrm{CO}_{2}$ deliveries and some did not. The trial began after the subject typed the letter that was shown on the screen. After each trial, the subjects gave a self-report of the unpleasantness of the trial and their level of anxiety. In Phase 2, both of the letters " $\mathrm{T}$ " and "N" were presented on the screen, and the subjects chose the trial type they would receive by typing one of the letters. Only the high-anxiety subjects chose the predictable trials significantly more 
than the unpredictable trials; the low-anxiety individuals chose both equally often. Ratings of anxiety and unpleasantness were not correlated with preference.

Yartz, Zvolensky, Bernstein, Bonn-Miller, and Lejuez (2008) conducted a similar study. Instead of tones to signal the type of trial, they used written instructions that were presented on the screen. Before each 90-s trial, college-student subjects were informed whether the trial was predictable (e.g. "Predictable Trial: You will receive $\mathrm{CO}_{2}$ during this trial" or "Predictable Trial: You will not receive $\mathrm{CO}_{2}$ during this trial") or unpredictable (e.g. "Unpredictable Trial: You will NOT be told whether or not you will receive $\mathrm{CO}_{2}$ on this trial"). After 24 trials, the subjects chose which trial they would receive next, either predictable or unpredictable. When given a choice between the two types of trials, most participants chose the predictable trial. In addition to assessing preference, after each trial, Yartz et al. also had the subjects report their level of anxiety after every trial, including the trials in which no $\mathrm{CO}_{2}$ was administered, or "air trials". Subjects reported equal levels of anxiety during trials in which $\mathrm{CO}_{2}$ was delivered, but they reported lower levels of anxiety during predictable air trials compared to unpredictable air trials. These data are consistent with the safety signal hypothesis discussed earlier - the predictable trials may be reinforcing because of the lower anxiety experienced during the safety period when the aversive event never occurs.

Grillon (2008) has assessed responses to predictable and unpredictable events in humans using a startle reflex methodology in his research on anxiety disorders. The startle is used to measure the context conditioning to predictable and unpredictable events by assessing the degree of startle to the environmental stimuli associated with each event. Context conditioning occurs when the environment associated with an aversive event, such as shock, comes to elicit a fear response, such as freezing or augmented startle. For example, a rat that has been presented with 
light-shock pairings in the operant chamber and whose startle is potentiated in the presence of the light may also develop potentiated startle in the operant chamber itself in the absence of any programmed stimuli. Context conditioning models sustained anxiety states and has been proposed as a model for phobic avoidance (agoraphobia) in panic disorder. Startle was selected as the primary dependent measure in Grillon's studies, because it can serve as a cross-species translational index of anxiety.

In one such study, Grillon, Baas, Lissek, Smith, and Milstein (2004), the acoustic startle stimulus was a 40-ms, 103-dB burst of white noise through headphones. The eyeblink reflex to the startle stimulus was recorded with electrodes placed under the left eye. In a typical study, subjects sat in front of a computer screen and were exposed to three different 2-min trial types: predictable shock, unpredictable shock, and no aversive stimulus. The 3 to $5 \mathrm{~mA}$ shock was delivered through electrodes on the subject's wrist. In the predictable shock condition, shocks only occurred when an 8-s cue (a geometric shape) was presented on the computer screen, and in the unpredictable shock condition, the shocks were not preceded by a cue. At the beginning of the trial, the type of trial was displayed on the computer screen (e.g. "no shock," "shock only during shape," or "shock at any time"). Acoustic startle stimuli were presented in each condition and between conditions. The amplitude of the startle reflex was higher in the unpredictable shock condition, suggesting that increased anxiety was experienced in the context in which unpredictable shocks could occur.

Grillon, Baas, Cornwell, and Johnson (2006) examined context conditioning using a virtual reality environment with three different rooms: a casino, a restaurant, and a bank. Each room was associated with predictable shock, unpredictable shock, or no shock, and the rooms were associated with different colored lamps. In the predictable room, the shock was always 
preceded with the onset of the lamp associated with the room. In the unpredictable room, the shock and the onset of the lamp were unpaired, and in the no shock room, no shocks were ever delivered. The virtual reality environment was presented on a screen, and during the acquisition phase the subjects watched the screen as they were moved through each of the rooms with the associated stimuli and shocks six times (like they were watching a movie). The startle stimulus was delivered every 18 to $26 \mathrm{~s}$. Startle was potentiated in the unpredictable room. In the final phase of the experiment, the shock and recording electrodes were removed and the subjects were told that a $\$ 10$ "money-box" was located in each room, and they were given a joystick and told that they could go to two of the rooms to retrieve them. Most subjects chose the no-shock and predictable rooms, avoiding the unpredictable room.

Signals are important under negative reinforcement contingencies, but signals also matter under schedules of positive reinforcement. The predictability of feedings has been an area of concern to those who work with captive animal populations. Feeding times in zoos are often signaled by their temporal regularity, and there is evidence to suggest that these predictable feedings can increase stereotypy, repetitive and excessive behaviors (such as pacing and selfinjury). For example, Boomsmith and Lambeth (1995) studied the effects of feeding times on behavior in a colony of chimpanzees. There were four existing social groups within the colony of chimpanzees, and two of the groups were fed according to a temporally predictable schedule and two of the groups were fed according to a temporally unpredictable schedule. Observational data were collected for several different types of behaviors, including abnormal behaviors and inactivity. Chimpanzees that were fed on the predictable schedule were significantly more inactive than those fed on the unpredictable schedule and engaged in more abnormal behavior, suggesting that, at least for chimpanzees, unpredictable feedings may be preferable. In a similar 
study, four leopard cats were fed according to an unpredictable schedule of multiple food deliveries after a baseline period in which food delivery occurred once a day according to a predictable schedule (Shepherdson, Carlstead, Mellen, \& Seidensticker, 1993). Feeding the cats on an unpredictable schedule decreased pacing and sleeping and increased grooming and exploratory behaviors.

\section{Timeout from Avoidance as an Animal Model of Phobic Behavior}

At first blush, the procedure used in the present experiment appears to be far removed from concerns beyond the basic research laboratory. While the present experimental arrangement was designed to assess basic behavioral processes relating to negative reinforcement, Stampfl (1987) has argued that the timeout-from-avoidance paradigm has the potential to serve as an animal model of human phobic behavior. He described how the timeout-from-avoidance preparation resolves many issues that have existed in comparing avoidance behavior inside and outside of the laboratory.

The timeout-from-avoidance model elegantly accounts for fundamental differences between the behavior of rats in avoidance preparations in the laboratory and phobic humans outside of the laboratory (Stampfl, 1987). In the laboratory, if a rat is avoiding shocks according to a shock-postponement schedule, and the shock generator is turned off and shocks are no longer delivered, avoidance responding will extinguish. In humans, however, avoidance behavior often persists for months or years without undergoing extinction (termed the "neurotic paradox" by Mowrer [1948]). Stampfl argues that a fundamental difference between the two environments is that the human is presented with serial stimuli that vary in the response effort necessary to avoid them. In contrast, in the operant chamber, the work requirement remains constant - a lever press at the beginning of the RS interval is identical to a lever press at the end of the RS interval. 
The human responds to stimuli early in the series, and these early responses prevent extinction to the primary aversive stimulus. For example, an individual with a bridge phobia who is planning a trip will respond by designing a route that detours around a particularly harrowing bridge.

Instead of making this response from the car, only a block or two away from the bridge, the individual responds early in the sequence of serial stimuli preceding the main source of aversiveness. In the laboratory, rats do not differentially respond to early stimuli in the sequence (Feild \& Boren, 1963), and their avoidance behavior eventually undergoes extinction.

In the timeout procedure, rats respond early and often to produce timeouts, similar to the avoidance behaviors of individuals with phobias described by Stampfl (1987). Not only do these responses early in the sequence decrease the likelihood of extinction, they also function as an additional reinforcing factor. When the individual with a bridge phobia makes an avoidance response by planning an alternate route on a trip to avoid the bridge, that avoidance response is followed by a period of time in which they are free from making another avoidance response. Essentially, planning the alternate route presents them with a timeout from avoidance. During this timeout, the individual may engage in positively reinforcing behaviors, and responding early in the sequence also allows them to maximize the amount of total time spend in timeout. For the rat, when a timeout is produced in the operant chamber, it can engage in alternative sources of reinforcement, such as grooming or napping.

In addition, with the timeout procedure, the behavior of rats under extinction models the behavior of phobic humans. Galizio (1999) assessed the resistance to extinction of timeout responding. In baseline, the procedure was similar to the baseline conditions in the present study - rats' responses postponed shock on one lever and produced 2-min timeouts on the other lever. In the extinction condition, the shock generator was turned off, but responses on the timeout 
lever still produced timeouts. Avoidance responding extinguished with the first few sessions, but timeout responding was highly resistant to extinction. For two of the rats, there was little change in timeout responding from the previous baseline after 20 to 30 sessions of extinction. One rat was still responding on the timeout lever after 100 2-hr sessions of extinction despite the absence of any shocks or avoidance responses during time-in. These patterns are similar to the behavior of humans with phobias who may go years without coming into contact with their feared stimulus but engage in avoidance behavior at a very high rate.

If the present experiments are examined from the perspective of timeout from avoidance as an animal model of human phobic behavior, then the shocks presented during timeout can be conceptualized as aversive events that may occur to a human phobic as a function of their timeout-from-avoidance responses. Planning the route for the bridge phobic results in the presentation of a timeout from avoidance, but during this timeout, they may receive disapproval from those with whom they are traveling. Do the aversive stimuli during the timeout decrease the reinforcing efficacy of the timeouts? If these aversive stimuli during the timeout are signaled or predictable, do the signals decrease their aversiveness?

\section{Future Directions}

The results of the present studies suggest that the reinforcing efficacy of the timeouts may be due to several factors, including shock-frequency reduction, response-effort reduction, and the role of the timeout as a signaled safety period. Future studies could investigate the role of signals in reducing the aversiveness of shocks in timeout. It is possible that much higher rate of shocks in timeout could occur without decreasing the reinforcing efficacy of the timeouts if those shocks are signaled rather than unsignaled. This could be investigated with a multiple schedule of timeout from avoidance in which, in one component, shocks in the timeouts are signaled, and in 
the other component, shocks are unsignaled. The probability of timeouts with shock could be raised across the conditions, and the point at which the reinforcing efficacy of the timeouts is degraded in the signaled and unsignaled shock components could be compared.

It is possible that unsignaled shocks are more aversive if signaled shocks occur first. In Experiment 3 of the present study, signals were added after exposure to unsignaled shocks in Experiment 1 - the rats were never exposed to signaled shocks first. Another potential experiment could examine signaling order by exposing the rats to signaled shocks in timeout in the first condition, then in a subsequent condition, removing the signals before the shocks in timeout. Greater decrements in timeout responding may occur if the rats are exposed to signaled shocks before unsignaled shocks. 


\section{References}

Abbott, B. B., \& Badia, P. (1979). Choice for signaled over unsignaled shock as a function of signal length. Journal of the Experimental Analysis of Behavior, 32, 409-417.

Abbott, B. B., \& Badia, P. (1984). Preference for signaled over unsignaled shock schedules: Ruling out asymmetry and response fixation as factors. Journal of the Experimental Analysis of Behavior, 41, 45-52.

Abreu, P. R., \& Santos, C. E. (2008). Behavioral models of depression: A critique of the emphasis on positive reinforcement. International Journal of Behavioral Consultation and Therapy, 4, 130-145.

Badia, P., Coker, C., \& Harsh, J. (1973). Choice of higher density signalled shock over lower density unsignalled shock. Journal of the Experimental Analysis of Behavior, 20, 47-55.

Badia, P., \& Culbertson, S. (1972). The relative aversiveness of signaled vs unsignalled escapable and inescapable shock. Journal of the Experimental Analysis of Behavior, 17, 463-471.

Badia, P., Culberston, S., \& Harsh, J. (1973). Choice of longer or stronger signalled shock over shorter or weaker unsignalled shock. Journal of the Experimental Analysis of Behavior, $19,25-32$.

Badia, P., Culbertson, S., \& Lewis, P. (1972). The relative aversiveness of signalled vs unsignalled avoidance. Journal of the Experimental Analysis of Behavior, 16. 113-121.

Badia, P., Harsh, J., \& Abbott, B. (1979). Choosing between predictable and unpredictable shock conditions: Data and theory. Psychological Bulletin, 86, 1107-1131.

Badia, P., Harsh, J., Coker, C. C., \& Abbott, B. (1976). Choice and the dependability of stimuli that predict shock and safety. Journal of the Experimental Analysis of Behavior, 26, 95- 
111.

Baron, A., DeWaard, R. J., \& Lipson, J. (1977). Increased reinforcement when timeout from avoidance includes access to a safe place. Journal of the Experimental Analysis of Behavior, 27, 479-494.

Baron, A., \& Perone, M. (2001). Explaining avoidance behavior: Two factors are still better than one. Journal of the Experimental Analysis of Behavior, 75, 357-361.

Baron, A. \& Trenholme, I. A. (1971). Response-dependent and response-independent timeout from an avoidance schedule. Journal of the Experimental Analysis of Behavior, $16,123-131$.

Bloomsmith, M. A., \& Lambeth, S. P. (1995). Effects of predictable versus unpredictable feeding schedules on chimpanzee behavior. Applied Animal Behaviour Science, 44, 6574.

Chorpita, B. F., \& Barlow, D. H. (1998). The development of anxiety: The role of control in the early environment. Psychological Bulletin, 124, 3-21.

Courtney, K., \& Perone, M. (1992). Reductions in shock frequency and response effort as factors in reinforcement by timeout from avoidance. Journal of the Experimental Analysis of Behavior, 58, 485-496.

de Villiers, P. A. (1974). The law of effect and avoidance: A quantitative relationship between response rate and shock-frequency reduction. Journal of the Experimental Analysis of Behavior, 21, 223-235.

Dinsmoor, J. A. (1977). Escape, avoidance, punishment: Where do we stand? Journal of the Experimental Analysis of Behavior, 28, 83-95. 
Falk, J. L. (1961). Production of polydipsia in normal rats by an intermittent food schedule. Science, 133, 195-196.

Feild, G. E., \& Boren, J. J. (1963). An adjusting avoidance procedure with multiple auditory and visual warning stimuli. Journal of the Experimental Analysis of Behavior, 6, 537-543.

Findley, J. D., \& Ames, L. L. (1965). A note on time out from avoidance with the chimpanzee. Journal of the Experimental Analysis of Behavior, 8, 419-423.

Fleshler, M., \& Hoffman, H. S. (1962). A progression for generating variable-interval schedules. Journal of the Experimental Analysis of Behavior, 5, 529-530.

Foa, E. B., Zinbarg, R., \& Rothbaum, B. O. (1992). Uncontrollability and unpredictability in post-traumatic stress: An animal model. Psychological Bulletin, 112, 218 - 238.

Foreman, A. (2009). Negative reinforcement by timeout from avoidance: The roles of shockfrequency reduction and response-effort reduction. Unpublished Master's Thesis, West Virginia University.

Galizio, M. (1999). Extinction of responding maintained by timeout from avoidance. Journal of the Experimental Analysis of Behavior, 71, 1-11.

Galizio, M., Hale, K. L., Liborio, M. O., \& Miller, M. (1993). Variable-ratio schedules of timeout from avoidance: Effects of anxiolytic drugs. Behavioral Pharmacology, 4, 487-493.

Galizio, M., \& Liborio, M. O. (1995). The effects of cocaine on behavior maintained by timeout from avoidance. Journal of Experimental Analysis Behavior, 63, 19-32.

Galizio, M. (1999). Extinction of responding maintained by timeout from avoidance. Journal of the Experimental Analysis of Behavior, 71, 1-11. 
Grillon, C. (2008). Models and mechanisms of anxiety: evidence from startle studies. Psychopharmacology, 199, 421-437.

Grillon, C., Bass, J. P., Cornwell, B., \& Johnson, L. (2006). Context conditioning and behavioral avoidance in a virtual reality environment: Effect of predictability. Biological Psychiatry, $60,752-759$.

Grillon, C., Bass, J. P., Lissek, S., Smith, K., \& Milstein, J. (2004). Anxious responses to predictable and unpredictable aversive events. Behavioral Neuroscience, 118, 916-924.

Grillon, C., Lissek, S., Rabin, S., McDowell, D., Dvir, S., \& Pine, D. S. (2008). Increased anxiety during anticipation of unpredictable but not predictable aversive stimuli as a psychophysiologic marker of panic disorder. The American Journal of Psychiatry, 165, 898-904.

Herrnstein, R. J., (1969). Method and theory in the study of avoidance. Psychological Review, 76, 49-69.

Herrnstein, R. J., \& Hineline, P. N. (1966). Negative reinforcement as shock-frequency reduction. Journal of the Experimental Analysis of Behavior, 5, 439-448.

Hineline, P. N. (1981). The several roles of stimuli in negative reinforcement. In P. Harzem \& M.D. Zeiler (Eds.), Advances in analysis of behavior: Vol 2. Predictability, correlation and contiguity (pp. 203-246). Chichester, England: Wiley.

Hymowitz, N. (1973). Comparisons between variable-interval and fixed-interval schedules of electric shock delivery. Journal of the Experimental Analysis of Behavior, 19, 101-111.

Hymowitz, N. (1976). Effects on responding of mixed and multiple schedules of signalled and unsignalled response-dependent electric-shock delivery. Journal of the Experimental Analysis of Behavior, 25, 321-326. 
Hymowitz, N. (1977). Effects of signalled and unsignalled electric-shock delivery on schedulecontrolled and schedule-induced behavior. The Psychological Record, 277, 715-731.

Iwata, B. A. (1987). Negative reinforcement in applied behavior analysis: An emerging technology. Journal of Applied Behavior Analysis, 20, 361-378.

Kanter, J. W., Busch, A. M., Weeks, C. E., \& Landes, S. J. (2008). The nature of clinical depression: Symptoms, syndromes, and behavior analysis. The Behavior Analyst, 31, 121.

Knapp, R. K., Kause, R. H. and Perkins, C. C. (1959). Immediate vs. delayed shock in Tmaze performance. Journal of Experimental Psychology, 58, 357-362.

Lejuez, C. W., Eifert, G. H., Zvolensky, M. J., \& Richards, J. B. (2000). Preference between onset predictable and unpredictable administrations of $20 \%$ carbon-dioxide-enriched air: Implications for better understanding the etiology and treatment of panic disorder. Journal of Experimental Psychology: Applied, 6, 349-358.

Lerman, D. C., \& Vorndran, C. M. (2002). On the status of knowledge for using punishment: Implications for treating behavior disorders. Journal of Applied Behavior Analysis, 35, 431-464.

Lewis, P., \& Gardner, E. T. (1977). The reliability of preference for signaled shock. Bulletin of the Psychonomic Society, 9, 135-138.

Lockard, J. S. (1963). Choice of a warning signal or no warning signal in an unavoidable shock situation. Journal of Comparative and Physiological Psychology, 56, 526-530.

MacDonald, L. (1973). The relative aversiveness of signalled versus unsignalled shockpunishment. Journal of the Experimental Analysis of Behavior, 20, 37-46.

MacDonald, L., \& Baron, A. (1973). A rate measure of the relative aversiveness of signalled vs 
unsignalled shock. Journal of the Experimental Analysis of Behavior, 19, 33-38.

Mineka, S., \& Zinbarg, R. (1996). Conditioning and ethological models of anxiety disorders:

Stress-in-dynamic-context anxiety models. In D. A. Hope (Ed.), Nebraska Symposium on Motivation: Vol 43. Perspectives on anxiety, panic, and fear (pp. 135-210). Lincoln: University of Nebraska Press.

Mowrer, O. H. (1948). Learning theory and the neurotic paradox. American Journal of Orthopsychiatry, 18, 571-610.

Nevin, J. A. (1974). Response strength in multiple schedules. Journal of the Experimental Analysis of Behavior, 21, 389-408.

Nevin, J. A. (1992). Behavioral contrast and behavioral momentum. Journal of Experimental Psychology: Animal Behavior Processes, 16, 298-305.

Perone, M. (2003). Negative effects of positive reinforcement. Journal of the Experimental Analysis of Behavior, 26, 1-14.

Perone M., \& Crawford, E. (1999). The role of intermittent shock-postponement in reinforcement by timeout from avoidance. Mexican Journal of Behavior Analysis, 25, 329-340.

Perone, M. \& Galizio, M. (1987). Variable-interval schedules of timeout from avoidance.

Journal of the Experimental Analysis of Behavior, 47, 97-113.

Posner, J., \& Baron, A. (1981, May). Progressive ratio schedules of positive and negative reinforcement in rats. Paper presented at the annual meeting of the Association for Behavior Analysis, Milwaukee, WI.

Seligman, M. E. (1968). Chronic fear produced by unpredictable electric shock. Journal of 
Comparative and Physiological Psychology, 66, 402-411.

Shepherdson, D. J., Carlstead, K., Mellen, J. D., \& Seidensticker, J. (1993). The influence of food presentation on the behavior of small cats in confined environments. Zoo Biology, 12, 203-216.

Sidman, M. (1953). Avoidance conditioning with brief shock and no exteroceptive warning signal. Science, 18, 157-158.

Sidman, M. (1962). Time out from avoidance as a reinforcer: A study of response interaction. Journal of the Experimental Analysis of Behavior, 5, 391-422.

Skinner, B. F. (1953). Science and Human Behavior. Macmillan; New York.

Stampfl, T. G. (1987). Theoretical implications of the neurotic paradox as a problem in behavior theory: An experimental resolution. The Behavior Analyst, 10, 161-173.

Verhave, T. (1962). The functional properties of a timeout from avoidance schedule. Journal of the Experimental Analysis of Behavior, 5, 391-422.

Yartz, A. R., Zvolensky, M. J., Bernstein, A., Bonn-Miller, M. O., \& Lejeuz, C. W. (2008). Panic-relevant predictability preferences: a laboratory test. Journal of Abnormal Psychology, 117, 242-246. 
Table 1

Experiment 1.

The programmed probability of a timeout with shock, the actual number of timeouts without and with shock, and the obtained probability of timeouts with shock for the last 10 sessions of each condition.

\begin{tabular}{|c|c|c|c|c|}
\hline \multirow[b]{2}{*}{ Rat } & \multirow{2}{*}{$\begin{array}{c}\text { Programmed } \\
p(\text { Timeout with } \\
\text { Shock) }\end{array}$} & \multicolumn{2}{|c|}{ Timeouts } & \multirow{2}{*}{$\begin{array}{c}\text { Obtained } \\
p \text { (Timeout } \\
\text { with Shock) }\end{array}$} \\
\hline & & $\begin{array}{l}\text { Without } \\
\text { Shock }\end{array}$ & $\begin{array}{l}\text { With } \\
\text { Shock }\end{array}$ & \\
\hline \multirow[t]{6}{*}{ AF5 } & .00 & 633 & 0 & .000 \\
\hline & .02 & 545 & 18 & .033 \\
\hline & .04 & 482 & 17 & .035 \\
\hline & .06 & 533 & 33 & .062 \\
\hline & .08 & 504 & 44 & .087 \\
\hline & .10 & 429 & 41 & .095 \\
\hline \multirow[t]{8}{*}{ AF6 } & .00 & 445 & 0 & .000 \\
\hline & .02 & 425 & 9 & .021 \\
\hline & .04 & 370 & 14 & .038 \\
\hline & .06 & 398 & 26 & .065 \\
\hline & .08 & 479 & 48 & .100 \\
\hline & .10 & 513 & 47 & .092 \\
\hline & .12 & 442 & 44 & .099 \\
\hline & .22 & 281 & 65 & .231 \\
\hline \multirow[t]{4}{*}{ AF12 } & .00 & 519 & 0 & .000 \\
\hline & .02 & 414 & 9 & .022 \\
\hline & .04 & 324 & 10 & .031 \\
\hline & .06 & 196 & 13 & .066 \\
\hline
\end{tabular}


Table 2

Experiment 1.

Summary of conditions, shock rates, avoidance response rates, discrimination indices ([avoidance responses in time-in and timeout / avoidance responses in time-in] x 100), and percentage of RS (response-shock) shocks avoided. Results are means of the last 10 stable sessions. Standard deviations are in parentheses.

\begin{tabular}{|c|c|c|c|c|c|c|c|c|c|c|c|c|c|c|}
\hline \multirow{3}{*}{$\frac{\text { Rat }}{\text { AF5 }}$} & \multirow{3}{*}{$\begin{array}{c}\text { Sessions } \\
25\end{array}$} & \multirow{3}{*}{$\begin{array}{c}p \text { (Timeout } \\
\text { with } \\
\text { shock) }\end{array}$} & \multicolumn{4}{|c|}{ Shocks per min } & & & \multicolumn{4}{|c|}{ Avoidance resp /min } & \multirow{3}{*}{$\begin{array}{c}\begin{array}{c}\text { Discrimination } \\
\text { Index }\end{array} \\
99\end{array}$} & \multirow{3}{*}{$\begin{array}{c}\% \text { RS } \\
\text { Shocks } \\
\text { Avoided } \\
98\end{array}$} \\
\hline & & & \multicolumn{2}{|c|}{ Time-in } & \multicolumn{2}{|c|}{ Timeout } & \multicolumn{2}{|c|}{$\begin{array}{l}\text { Timeout } \\
\text { resp/ min }\end{array}$} & \multicolumn{2}{|c|}{ Time-in } & \multicolumn{2}{|c|}{ Timeout } & & \\
\hline & & & 0.17 & $(0.42)$ & 0.00 & $(0.00)$ & 3.68 & $(0.59)$ & 3.97 & $(0.51)$ & 0.02 & $(0.04)$ & & \\
\hline & 22 & 0.02 & 0.07 & $(0.09)$ & 0.02 & $(0.01)$ & 2.71 & $(0.60)$ & 4.22 & $(0.36)$ & 0.03 & $(0.07)$ & 99 & 97 \\
\hline & 23 & 0.04 & 0.03 & $(0.01)$ & 0.02 & $(0.01)$ & 1.86 & $(0.39)$ & 4.09 & $(0.58)$ & 0.00 & $(0.00)$ & 99 & 99 \\
\hline & 35 & 0.06 & 0.23 & $(0.14)$ & 0.03 & $(0.01)$ & 2.12 & $(0.33)$ & 4.37 & $(0.45)$ & 0.01 & $(0.01)$ & 99 & 88 \\
\hline & 67 & 0.08 & 0.18 & $(0.15)$ & 0.04 & $(0.02)$ & 2.04 & $(0.47)$ & 4.30 & $(0.31)$ & 0.10 & $(0.05)$ & 96 & 91 \\
\hline & 31 & 0.10 & 0.12 & $(0.15)$ & 0.05 & $(0.02)$ & 1.49 & $(0.22)$ & 3.74 & $(0.22)$ & 0.08 & $(0.04)$ & 97 & 95 \\
\hline \multirow[t]{8}{*}{ AF6 } & 35 & 0.00 & 0.27 & $(0.18)$ & 0.00 & $(0.00)$ & 1.24 & $(0.21)$ & 5.86 & $(0.34)$ & 0.02 & $(0.02)$ & 99 & 87 \\
\hline & 35 & 0.02 & 0.32 & $(0.13)$ & 0.01 & $(0.01)$ & 1.37 & $(0.34)$ & 5.01 & $(0.41)$ & 0.04 & $(0.04)$ & 99 & 85 \\
\hline & 25 & 0.04 & 0.27 & $(0.20)$ & 0.19 & $(0.01)$ & 1.10 & $(0.30)$ & 4.45 & $(0.47)$ & 0.01 & $(0.01)$ & 99 & 87 \\
\hline & 35 & 0.06 & 0.60 & $(0.23)$ & 0.03 & $(0.02)$ & 1.36 & $(0.11)$ & 4.45 & $(0.29)$ & 0.07 & $(0.06)$ & 97 & 71 \\
\hline & 31 & 0.08 & 0.70 & $(0.29)$ & 0.05 & $(0.02)$ & 1.47 & $(0.15)$ & 5.01 & $(0.57)$ & 0.17 & $(0.07)$ & 94 & 65 \\
\hline & 48 & 0.10 & 0.69 & $(0.41)$ & 0.05 & $(0.01)$ & 2.01 & $(0.36)$ & 4.87 & $(0.64)$ & 0.18 & $(0.06)$ & 94 & 65 \\
\hline & 22 & 0.12 & 0.72 & $(0.41)$ & 0.05 & $(0.03)$ & 1.93 & $(0.62)$ & 5.25 & $(0.65)$ & 0.18 & $(0.13)$ & 94 & 64 \\
\hline & 29 & 0.22 & 0.01 & $(0.01)$ & 0.12 & $(0.04)$ & 0.97 & $(0.44)$ & 4.45 & $(0.30)$ & 0.03 & $(0.03)$ & 99 & 99 \\
\hline \multirow[t]{4}{*}{ AF12 } & 26 & 0.00 & 0.39 & $(0.16)$ & 0.00 & $(0.00)$ & 2.44 & $(0.44)$ & 8.77 & $(1.02)$ & 0.01 & $(0.01)$ & 99 & 89 \\
\hline & 33 & 0.02 & 0.08 & $(0.09)$ & 0.01 & $(0.01)$ & 1.66 & $(0.49)$ & 12.53 & $(0.86)$ & 0.29 & $(0.44)$ & 97 & 98 \\
\hline & 31 & 0.04 & 0.09 & $(0.04)$ & 0.01 & $(0.01)$ & 1.77 & $(0.98)$ & 11.03 & $(2.22)$ & 0.04 & $(0.07)$ & 99 & 97 \\
\hline & 50 & 0.06 & 0.04 & $(0.02)$ & 0.04 & $(0.04)$ & 1.05 & $(0.76)$ & 10.97 & $(1.48)$ & 0.00 & $(0.00)$ & 99 & 99 \\
\hline
\end{tabular}


Table 3

Experiment 2.

Summary of conditions (shock free, local yoking, and random (R.) local yoking), shock rates, response rates, discrimination (discr.) indices (avoidance

responses in time-in / avoidance responses in time-in + avoidance responses in timeout), and percentage of RS (response-shock) shocks avoided. Results are

means of the last 10 stable sessions. Standard deviations are in parentheses.

\begin{tabular}{|c|c|c|c|c|c|c|c|c|c|c|c|c|c|c|}
\hline \multirow{3}{*}{$\frac{\text { Rat }}{\text { AF2 }}$} & \multirow{3}{*}{$\frac{\text { Sessions }}{30}$} & \multirow{3}{*}{$\begin{array}{c}\text { Condition } \\
\text { Shock Free }\end{array}$} & \multicolumn{4}{|c|}{ Shocks per min } & \multirow{2}{*}{\multicolumn{2}{|c|}{$\begin{array}{l}\text { Timeout resp/ } \\
\min \end{array}$}} & \multicolumn{4}{|c|}{ Avoidance resp /min } & \multirow{2}{*}{$\begin{array}{l}\text { Discr. } \\
\text { Index }\end{array}$} & \multirow{2}{*}{$\begin{array}{c}\% \text { RS } \\
\text { Shocks } \\
\text { Avoided }\end{array}$} \\
\hline & & & \multicolumn{2}{|c|}{ Time-in } & \multicolumn{2}{|c|}{ Timeout } & & & \multicolumn{2}{|c|}{ Time-in } & \multicolumn{2}{|c|}{ Timeout } & & \\
\hline & & & 0.09 & $(0.05)$ & 0.00 & $(0.00)$ & 2.10 & $(0.44)$ & 3.49 & $(0.31)$ & 0.01 & $(0.02)$ & 99 & 96 \\
\hline & 23 & Local Yoking & 0.11 & $(0.05)$ & 0.01 & $(0.01)$ & 2.21 & $(0.32)$ & 3.53 & $(0.28)$ & 0.01 & $(0.02)$ & 99 & 97 \\
\hline & 61 & Shock Free & 0.08 & $(0.09)$ & 0.00 & $(0.00)$ & 2.73 & $(0.25)$ & 3.39 & $(0.19)$ & 0.03 & $(0.02)$ & 98 & 97 \\
\hline & 24 & R. Local Yoking & 0.06 & $(0.03)$ & 0.01 & $(0.02)$ & 2.46 & $(0.36)$ & 4.01 & $(0.32)$ & 0.05 & $(0.02)$ & 98 & 98 \\
\hline & 27 & Shock Free & 0.06 & $(0.03)$ & 0.00 & $(0.00)$ & 2.71 & $(0.17)$ & 3.61 & $(0.29)$ & 0.03 & $(0.02)$ & 99 & 97 \\
\hline & 21 & R. Local Yoking & 0.09 & $(0.04)$ & 0.02 & $(0.01)$ & 1.75 & $(0.34)$ & 3.66 & $(0.16)$ & 0.05 & $(0.03)$ & 98 & 96 \\
\hline & 23 & Shock Free & 0.23 & $(0.26)$ & 0.00 & $(0.00)$ & 2.94 & $(0.39)$ & 3.36 & $(0.48)$ & 0.02 & $(0.02)$ & 99 & 89 \\
\hline & 20 & Local Yoking & 0.07 & $(0.04)$ & 0.02 & $(0.01)$ & 2.53 & $(0.41)$ & 3.60 & $(0.23)$ & 0.03 & $(0.01)$ & 99 & 97 \\
\hline \multirow[t]{8}{*}{$\mathrm{AF} 4$} & 29 & Shock Free & 0.27 & $(0.21)$ & 0.00 & $(0.00)$ & 2.86 & $(0.48)$ & 5.45 & $(0.44)$ & 0.06 & $(0.04)$ & 98 & 89 \\
\hline & 26 & R. Local Yoking & 0.16 & $(0.12)$ & 0.07 & $(0.10)$ & 4.22 & $(0.50)$ & 5.41 & $(0.49)$ & 0.13 & $(0.12)$ & 96 & 94 \\
\hline & 29 & Shock Free & 0.39 & $(0.23)$ & 0.00 & $(0.00)$ & 3.85 & $(0.49)$ & 5.37 & $(0.43)$ & 0.05 & $(0.03)$ & 98 & 82 \\
\hline & 24 & Local Yoking & 0.16 & $(0.06)$ & 0.10 & $(0.06)$ & 3.53 & $(0.44)$ & 5.96 & $(0.41)$ & 0.24 & $(0.16)$ & 93 & 93 \\
\hline & 36 & Shock Free & 0.49 & $(0.18)$ & 0.00 & $(0.00)$ & 3.87 & $(0.35)$ & 6.06 & $(0.47)$ & 0.14 & $(0.08)$ & 95 & 76 \\
\hline & 22 & R. Local Yoking & 0.13 & $(0.07)$ & 0.15 & $(0.09)$ & 4.33 & $(0.34)$ & 7.16 & $(1.04)$ & 0.34 & $(0.11)$ & 90 & 94 \\
\hline & 21 & Shock Free & 0.18 & $(0.11)$ & 0.00 & $(0.00)$ & 4.22 & $(0.86)$ & 6.76 & $(2.47)$ & 0.13 & $(0.12)$ & 95 & 91 \\
\hline & 23 & Local Yoking & 0.10 & $(0.03)$ & 0.08 & $(0.03)$ & 3.76 & $(0.25)$ & 5.07 & $(0.51)$ & 0.20 & $(0.09)$ & 92 & 96 \\
\hline \multirow[t]{8}{*}{ AF7 } & 29 & Shock Free & 0.21 & $(0.11)$ & 0.00 & $(0.00)$ & 2.14 & $(0.28)$ & 4.20 & $(0.28)$ & 0.01 & $(0.01)$ & 100 & 93 \\
\hline & 28 & R. Local Yoking & 0.05 & $(0.04)$ & 0.01 & $(0.01)$ & 1.44 & $(0.37)$ & 3.82 & $(0.43)$ & 0.01 & $(0.01)$ & 100 & 99 \\
\hline & 21 & Shock Free & 0.02 & $(0.02)$ & 0.00 & $(0.00)$ & 2.19 & $(0.26)$ & 3.87 & $(0.31)$ & 0.01 & $(0.01)$ & 100 & 99 \\
\hline & 20 & Local Yoking & 0.05 & $(0.03)$ & 0.00 & $(0.01)$ & 2.39 & $(0.23)$ & 4.16 & $(0.40)$ & 0.01 & $(0.01)$ & 100 & 99 \\
\hline & 56 & Shock Free & 0.05 & $(0.00)$ & 0.00 & $(0.00)$ & 2.46 & $(0.38)$ & 3.76 & $(0.44)$ & 0.01 & $(0.01)$ & 100 & 98 \\
\hline & 27 & R. Local Yoking & 0.04 & $(0.03)$ & 0.01 & $(0.02)$ & 2.95 & $(0.46)$ & 4.08 & $(0.47)$ & 0.00 & $(0.00)$ & 100 & 99 \\
\hline & 24 & Shock Free & 0.28 & $(0.12)$ & 0.00 & $(0.00)$ & 3.48 & $(0.63)$ & 4.86 & $(0.86)$ & 0.01 & $(0.01)$ & 99 & 86 \\
\hline & 21 & Local Yoking & 0.17 & $(0.07)$ & 0.11 & $(0.09)$ & 2.11 & $(0.69)$ & 5.36 & $(0.55)$ & 0.31 & $(0.31)$ & 91 & 92 \\
\hline
\end{tabular}


Table 3 Continued.

\begin{tabular}{|c|c|c|c|c|c|c|c|c|c|c|c|c|c|c|}
\hline \multirow{3}{*}{$\frac{\text { Rat }}{\text { AF10 }}$} & \multirow{3}{*}{$\frac{\text { Sessions }}{20}$} & \multirow{3}{*}{$\begin{array}{c}\text { Condition } \\
\text { Shock Free }\end{array}$} & \multicolumn{4}{|c|}{ Shocks per min } & \multirow{2}{*}{\multicolumn{2}{|c|}{$\begin{array}{l}\text { Timeout resp/ } \\
\text { min }\end{array}$}} & \multicolumn{4}{|c|}{ Avoidance resp /min } & \multirow{2}{*}{$\begin{array}{l}\text { Discr. } \\
\text { Index }\end{array}$} & \multirow{3}{*}{$\begin{array}{c}\begin{array}{c}\% \text { RS } \\
\text { Shocks } \\
\text { Avoided }\end{array} \\
70\end{array}$} \\
\hline & & & \multicolumn{2}{|c|}{ Time-in } & \multicolumn{2}{|c|}{ Timeout } & & & \multicolumn{2}{|c|}{ Time-in } & \multicolumn{2}{|c|}{ Timeout } & & \\
\hline & & & 0.70 & $(0.30)$ & 0.00 & $(0.00)$ & 2.08 & $(0.23)$ & 2.71 & $(0.42)$ & 0.06 & $(0.04)$ & 96 & \\
\hline & 26 & Local Yoking & 0.26 & $(0.18)$ & 0.02 & $(0.02)$ & 1.41 & $(0.21)$ & 4.64 & $(0.34)$ & 0.03 & $(0.02)$ & 99 & 93 \\
\hline & 33 & Shock Free & 0.37 & $(0.73)$ & 0.00 & $(0.00)$ & 1.79 & $(0.12)$ & 4.33 & $(0.50)$ & 0.05 & $(0.03)$ & 98 & 83 \\
\hline & 24 & R. Local Yoking & 0.17 & $(0.04)$ & 0.01 & $(0.01)$ & 1.21 & $(0.09)$ & 4.33 & $(0.09)$ & 0.04 & $(0.02)$ & 99 & 92 \\
\hline & 26 & Shock Free & 0.19 & $(0.13)$ & 0.00 & $(0.00)$ & 1.67 & $(0.22)$ & 4.21 & $(0.23)$ & 0.07 & $(0.02)$ & 97 & 92 \\
\hline & 24 & R. Local Yoking & 0.11 & $(0.08)$ & 0.01 & $(0.01)$ & 1.91 & $(0.27)$ & 4.27 & $(0.43)$ & 0.07 & $(0.02)$ & 97 & 95 \\
\hline & 32 & Shock Free & 0.14 & $(0.06)$ & 0.00 & $(0.00)$ & 1.92 & $(0.48)$ & 3.87 & $(0.43)$ & 0.04 & $(0.02)$ & 98 & 94 \\
\hline & 21 & Local Yoking & 0.06 & $(0.05)$ & 0.01 & $(0.01)$ & 2.17 & $(0.28)$ & 3.67 & $(0.18)$ & 0.02 & $(0.20)$ & 99 & 97 \\
\hline
\end{tabular}


Table 4

Experiment 3.

Summary of conditions, shock rates, response rates, discrimination indices ([avoidance responses in time-in / avoidance responses in time-in +

avoidance responses in timeout] x 100), and the percentage of RS shocks avoided. Results are means of the last 10 stable sessions. Standard deviations are in parentheses.

\begin{tabular}{|c|c|c|c|c|c|c|c|c|c|c|c|c|c|c|}
\hline \multirow{2}{*}{$\frac{\text { Rat }}{\text { AF5 }}$} & \multirow{2}{*}{$\begin{array}{c}\text { Sessions } \\
31\end{array}$} & \multirow{2}{*}{$\begin{array}{c}\begin{array}{c}\text { Type of } \\
\text { Shock }\end{array} \\
\text { Unsignaled }\end{array}$} & \multicolumn{4}{|c|}{ Shocks per min } & \multicolumn{2}{|c|}{$\begin{array}{l}\text { Timeout resp/ } \\
\text { min }\end{array}$} & \multicolumn{4}{|c|}{ Avoidance resp / $\mathrm{min}$} & \multirow{2}{*}{$\begin{array}{c}\begin{array}{c}\text { Discrimin- } \\
\text { ation } \\
\text { Index }\end{array} \\
97\end{array}$} & \multirow{2}{*}{$\begin{array}{c}\begin{array}{c}\% \text { RS } \\
\text { Shocks } \\
\text { Avoided }\end{array} \\
95\end{array}$} \\
\hline & & & 0.12 & $(0.15)$ & 0.05 & $(0.02)$ & 1.49 & $(0.22)$ & 3.74 & $(0.22)$ & 0.08 & $(0.04)$ & & \\
\hline & 21 & Unsignaled & 0.35 & $(0.14)$ & 0.05 & $(0.01)$ & 2.06 & $(0.26)$ & 3.43 & $(0.28)$ & 0.17 & $(0.07)$ & 91 & 82 \\
\hline & 20 & Signaled & 0.05 & $(0.04)$ & 0.05 & $(0.02)$ & 2.66 & $(0.31)$ & 4.40 & $(0.32)$ & 0.12 & $(0.11)$ & 95 & 98 \\
\hline \multirow[t]{3}{*}{ AF6 } & 29 & Unsignaled & 0.01 & $(0.01)$ & 0.12 & $(0.04)$ & 0.97 & $(0.44)$ & 4.45 & $(0.30)$ & 0.03 & $(0.03)$ & 99 & 99 \\
\hline & 23 & Signaled & 0.01 & $(0.01)$ & 0.11 & $(0.03)$ & 1.72 & $(0.42)$ & 4.47 & $(0.62)$ & 0.14 & $(0.26)$ & 99 & 99 \\
\hline & 20 & Unsignaled & 0.00 & $(0.01)$ & 0.09 & $(0.04)$ & 1.17 & $(0.50)$ & 4.56 & $(0.56)$ & 0.01 & $(0.01)$ & 99 & 99 \\
\hline AF12 & 50 & Unsignaled & 0.04 & $(0.02)$ & 0.04 & $(0.04)$ & 1.05 & $(0.76)$ & 10.97 & $(1.48)$ & 0.00 & $(0.00)$ & 99 & 99 \\
\hline
\end{tabular}




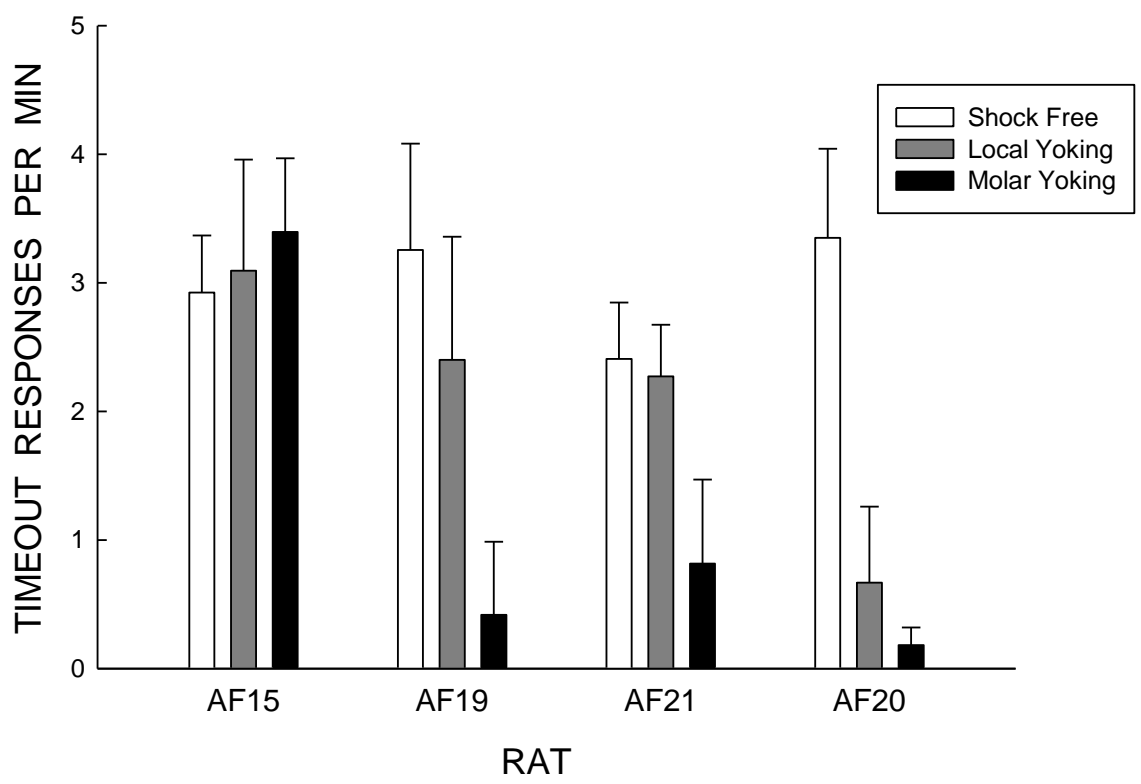

Figure 1. From Foreman (2009). Mean timeout response rates from the stable sessions of each condition. Error bars represent one standard deviation. 


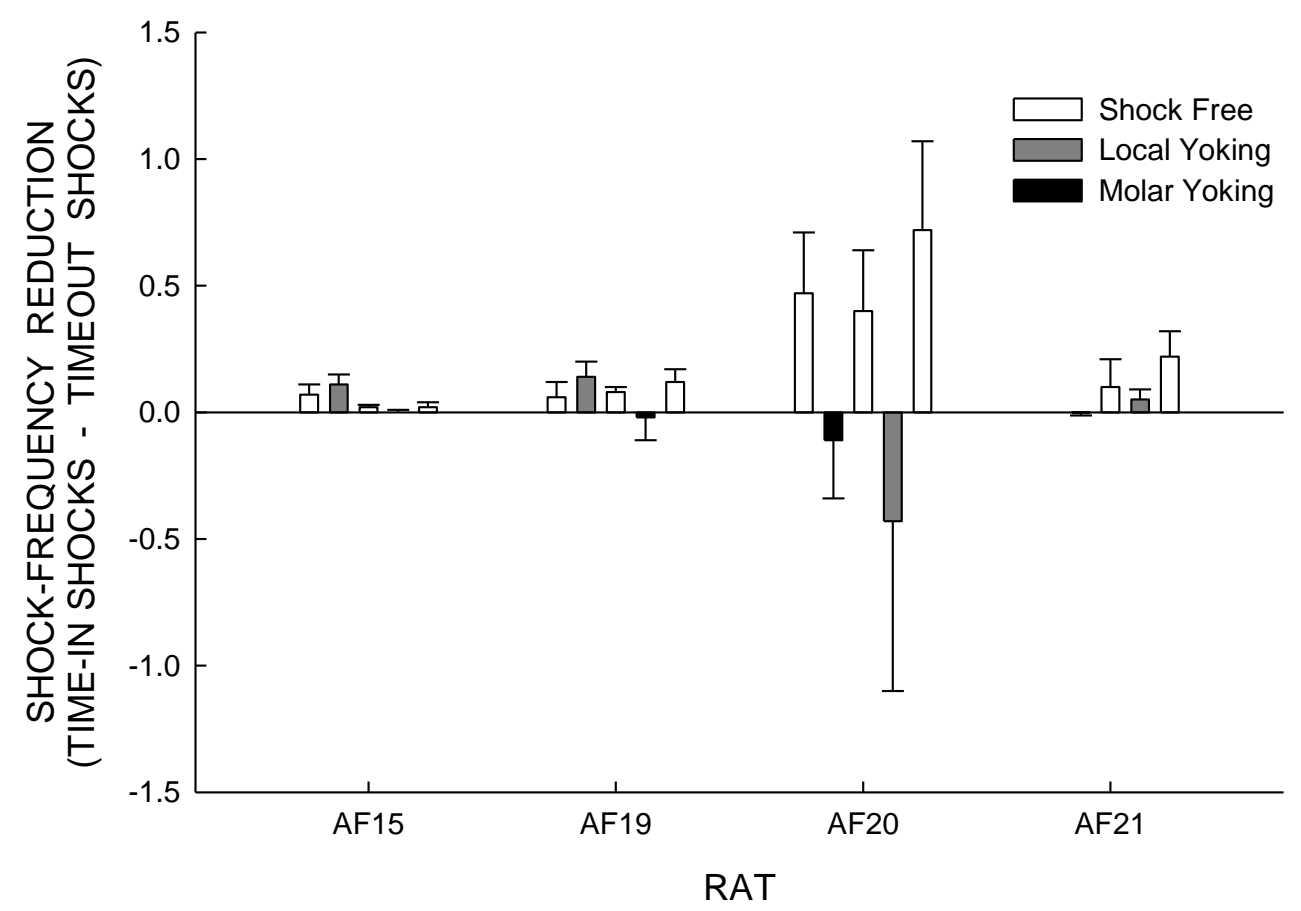

Figure 2. From Foreman (2009) Mean shock-frequency reduction (the timeout shock rate subtracted from the time-in shock rate) of the last six sessions of each condition. Error bars represent one standard deviation. 


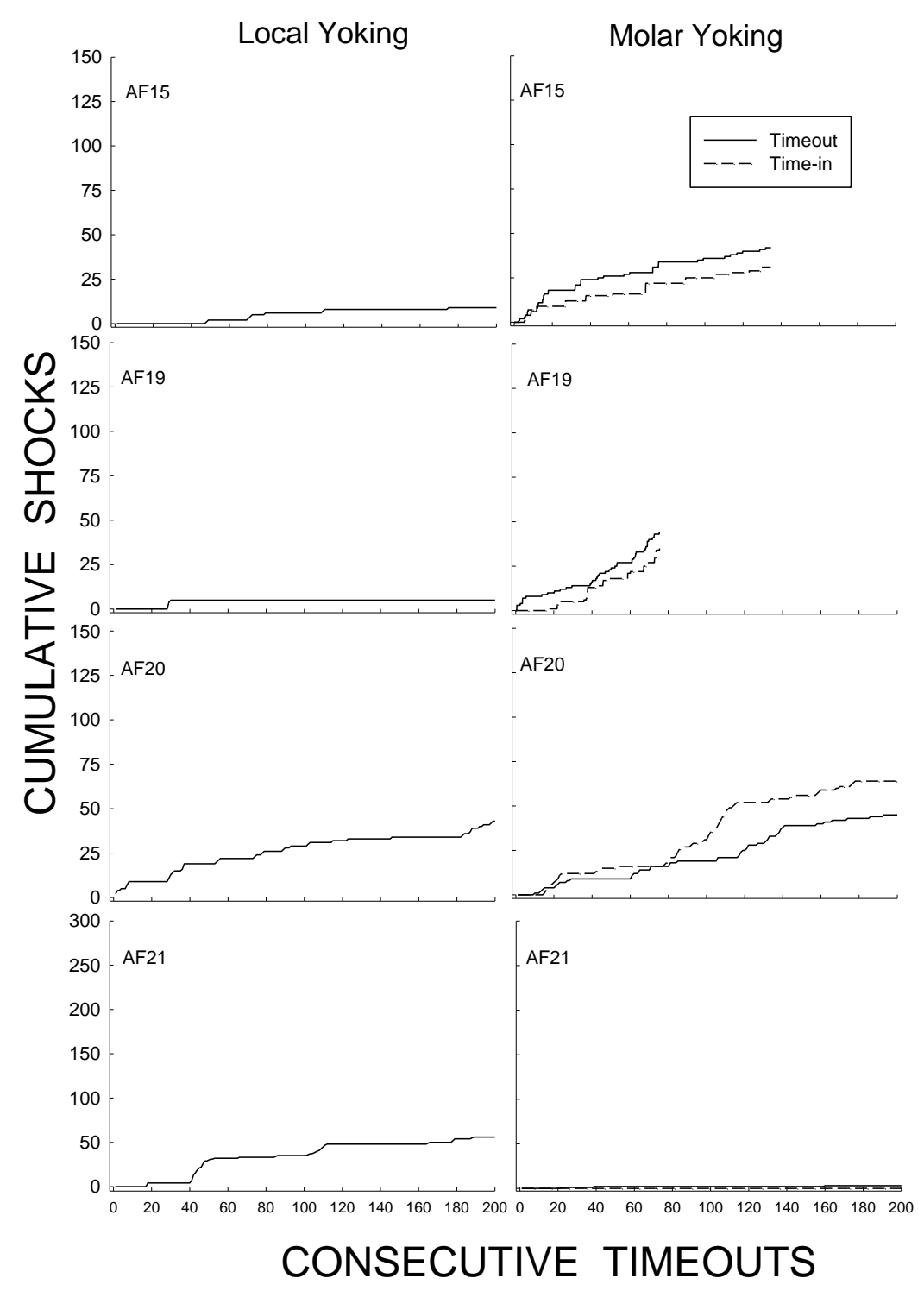

Figure 3. From Foreman (2009). Cumulative shocks during time-in (dotted line) and timeout (sold line) across the first 200 consecutive timeouts in Local Yoking (left column) and Molar Yoking (right column). 


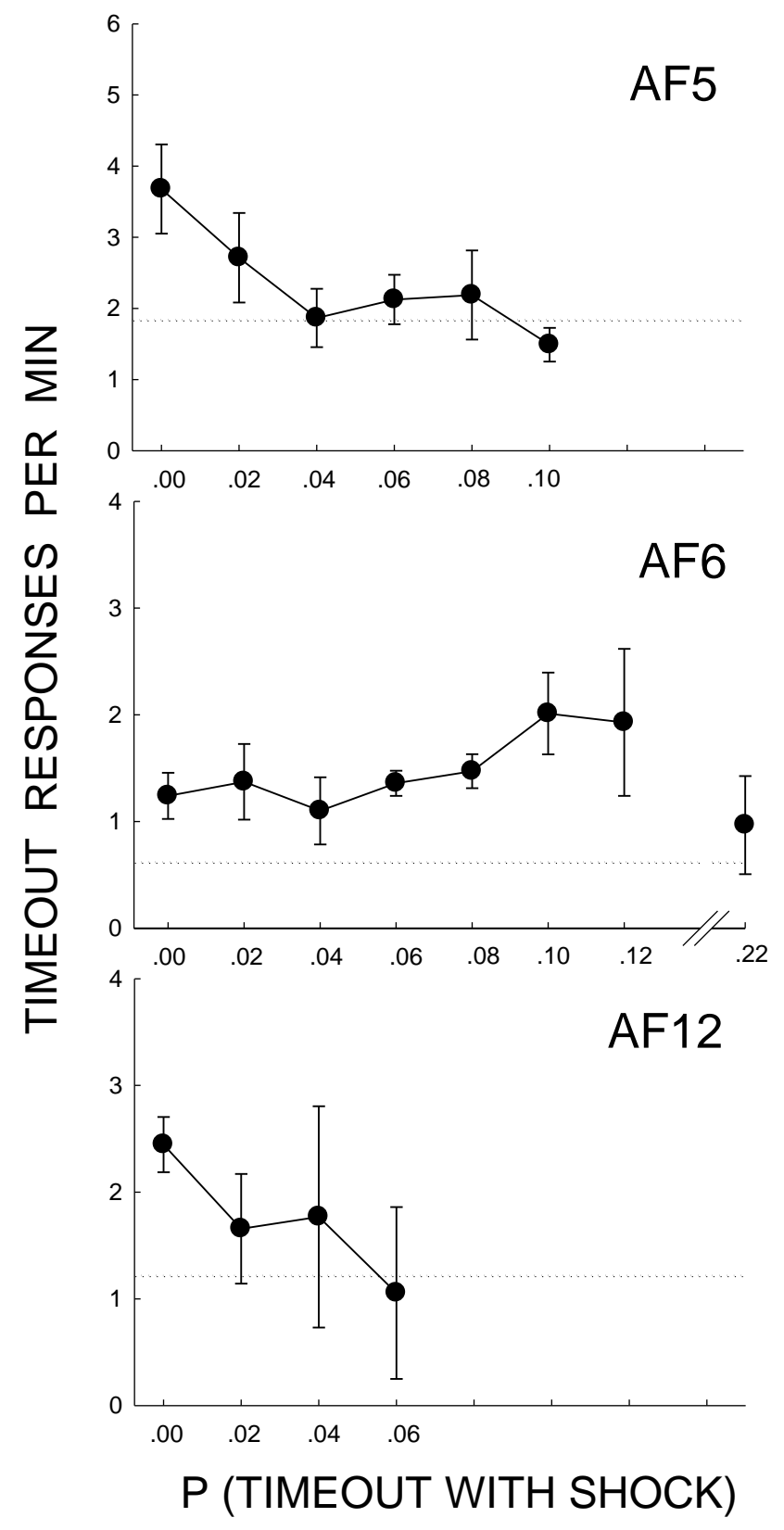

Figure 4. Experiment 1. Mean timeout responses per min for the last 10 sessions of each condition. Error bars represent one standard deviation. The dotted line shows 50\% of baseline responding. Note the different axis for Rat AF5. 


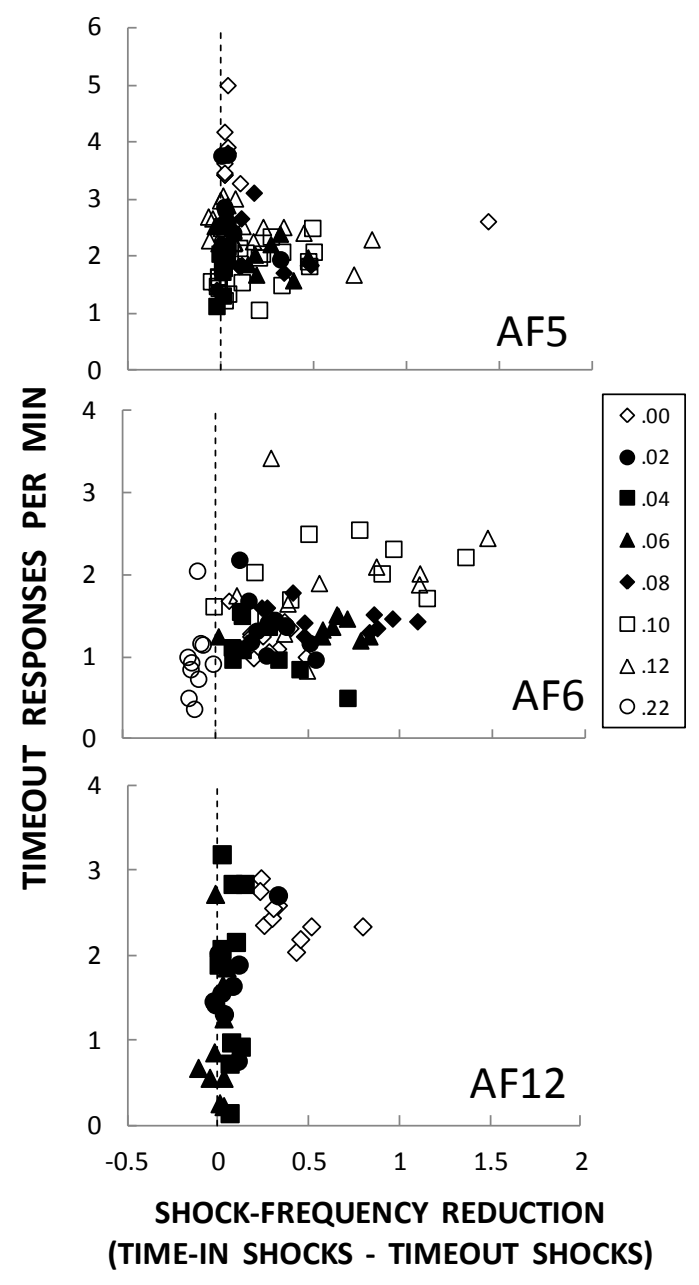

Figure 5. Experiment 1. Timeout responding plotted against shock- frequency reduction. Each data point represents performance in one of the last 10 sessions of each condition. Shockfrequency reduction values were calculated by subtracting the mean timeout shock rate for a session from the mean time-in shock rate for that session. Note the different axis for Rat AF5. 


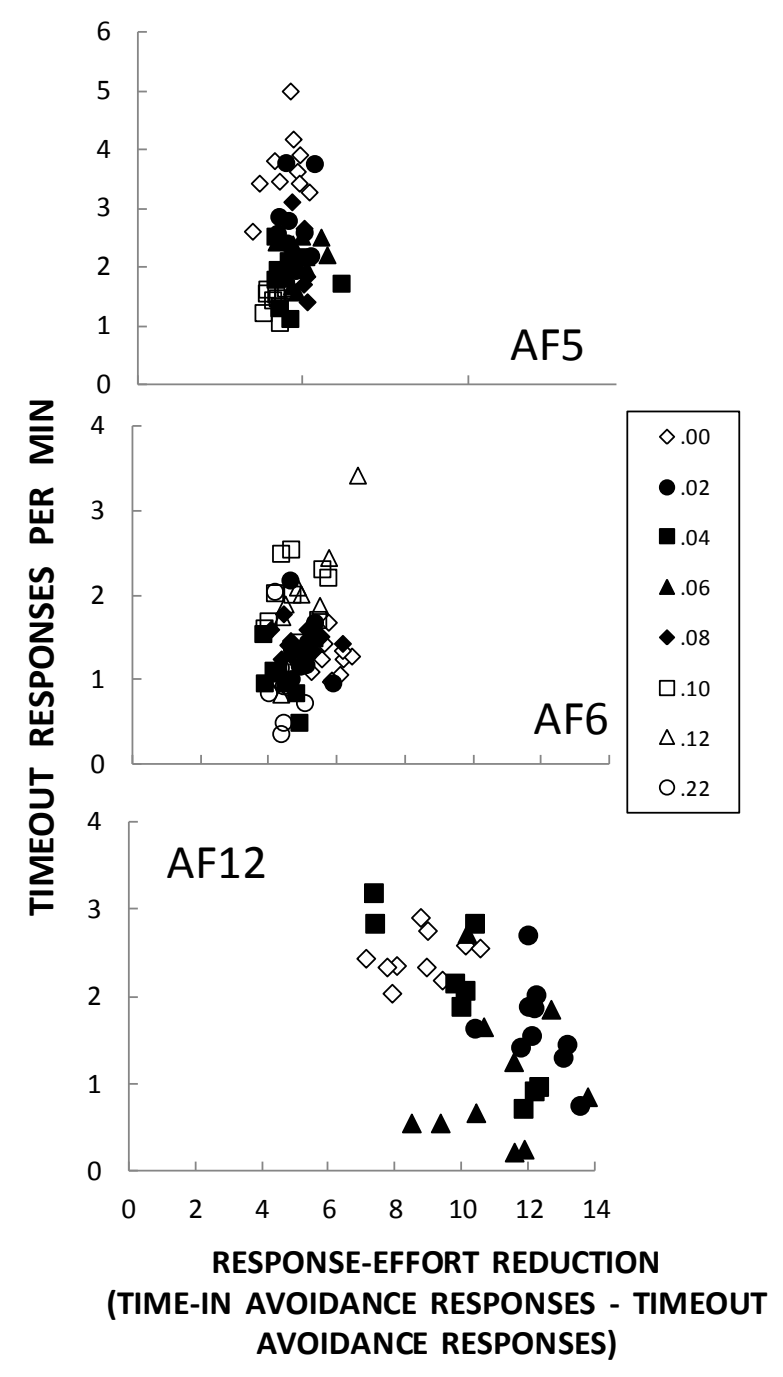

Figure 6. Experiment 1. Timeout responding plotted against response-effort reduction. Each data point represents performance in one of the last 10 sessions of each condition. Response-effort reduction values were calculated by subtracting the mean timeout avoidance response rate for a session from the mean time-in avoidance response rate for that session. Note the different axis for Rat AF5. 


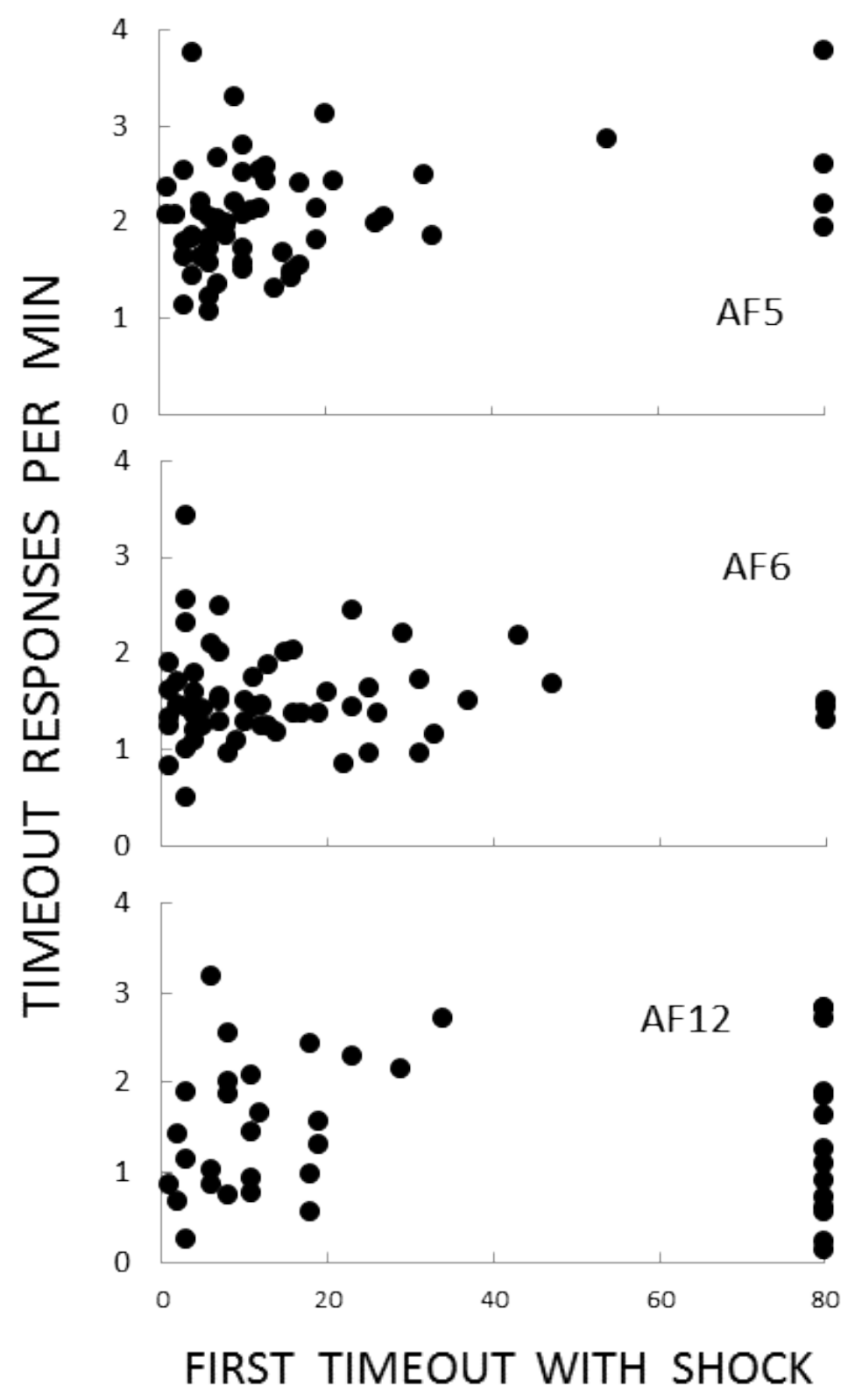

Figure 7. Experiment 1. Mean timeout responding plotted against the first timeout with shock in a session. Each data point represents mean performance in one of the last 10 sessions of each condition. If there were no shocks in a session, the data point was placed at 80 on the x-axis (see explanation in text). 


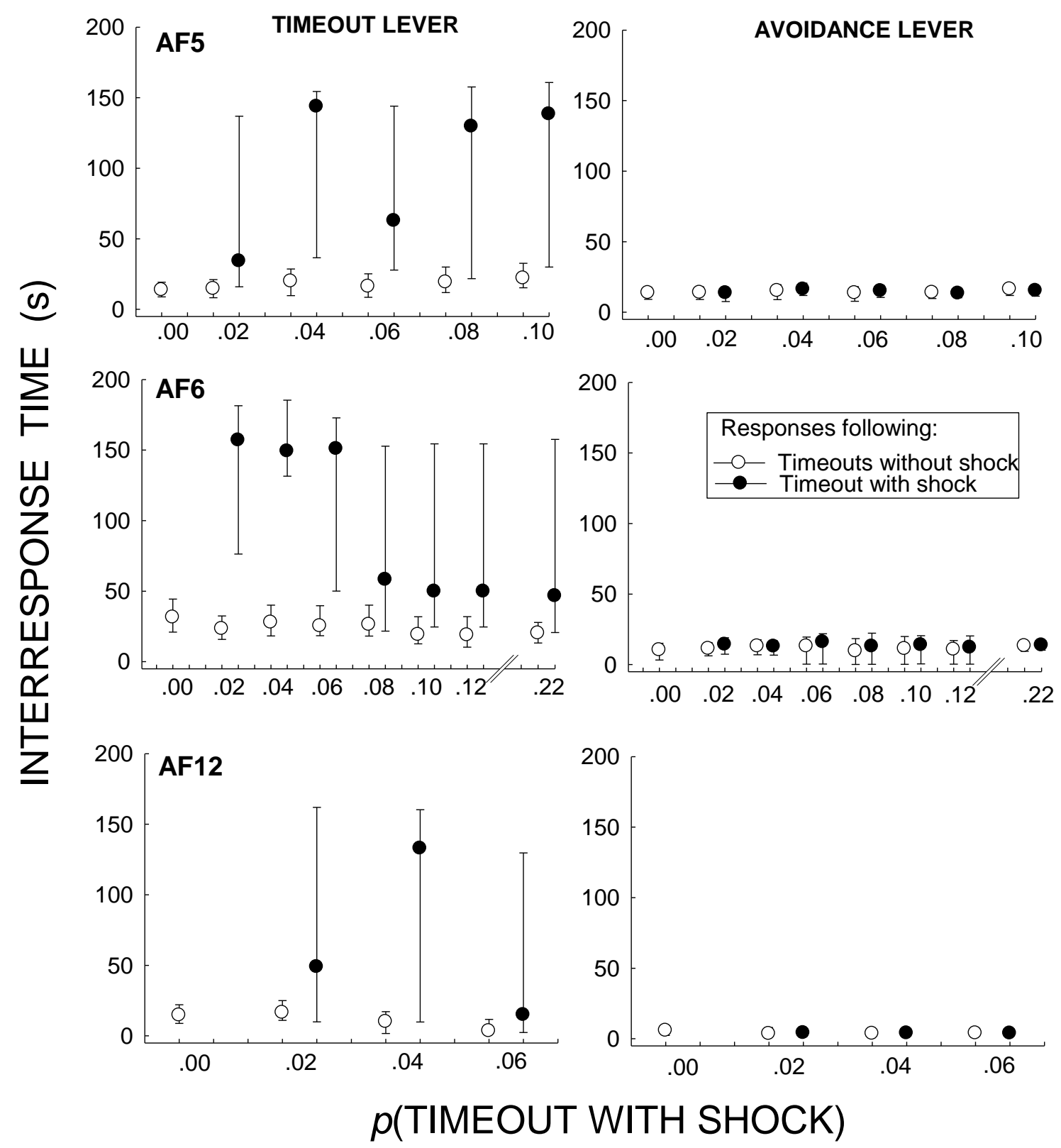

Figure 8. Experiment 1. Median intterresponse times on the timeout (left column) and avoidance (right column) levers for timeouts without shock (open circles) and with shock (filled circles). Data are from the last 10 sessions of each condition. Error bars represent the interquartile ranges. 


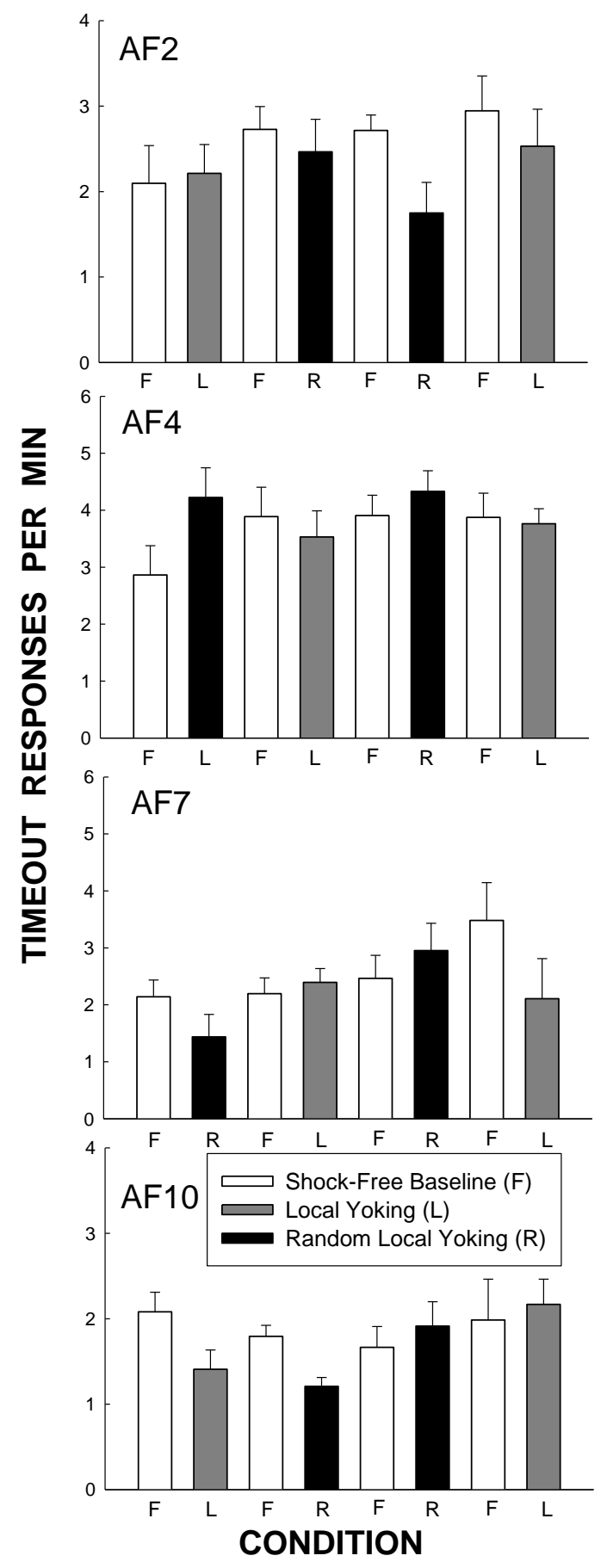

Figure 9. Experiment 2. Mean timeout responses per min for the last 10 sessions of each condition. Error bars represent one standard deviation. Note the different axes. 


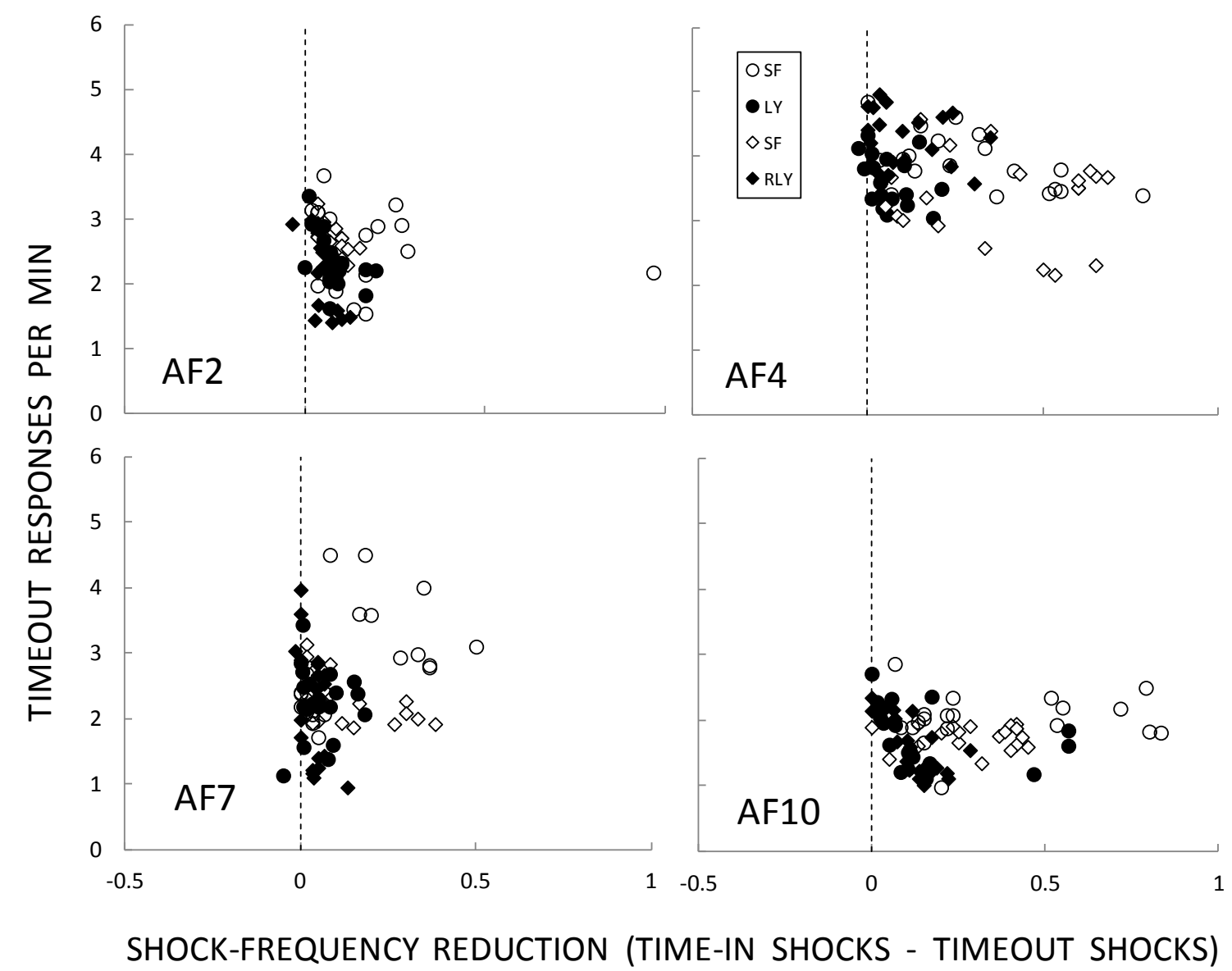

Figure 10. Experiment 2. Timeout responding plotted against shock-frequency reduction. Each data point represents performance in one of the last 10 sessions of each condition. Shockfrequency reduction values were calculated by subtracting the mean timeout shock rate for a session from the mean time-in shock rate for that session. 


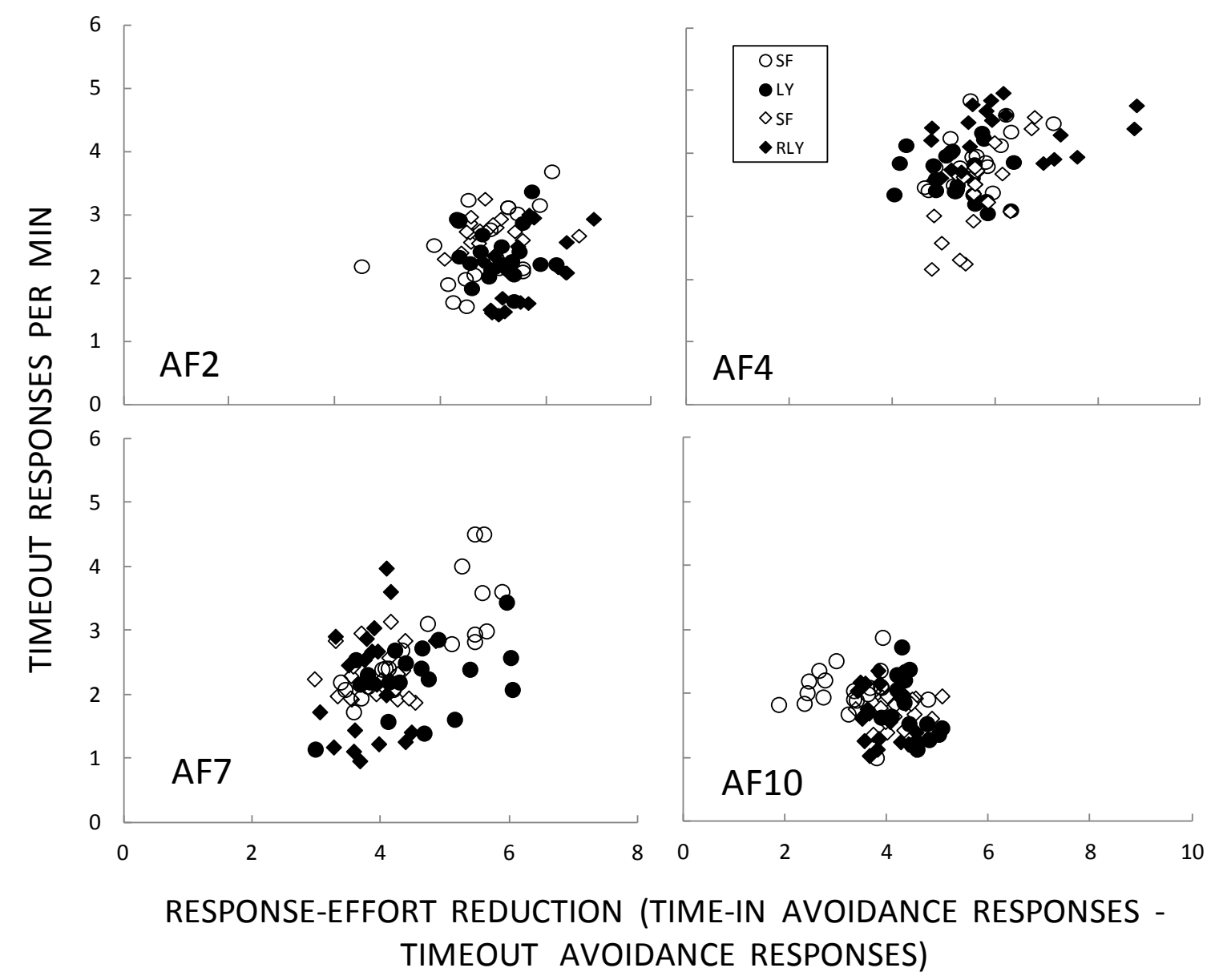

Figure 11. Experiment 2. Timeout responding plotted against response-effort reduction. Each data point represents performance in one of the last 10 sessions of each condition. Responseeffort reduction values were calculated by subtracting the mean timeout avoidance response rate for a session from the mean time-in avoidance response rate for that session. 


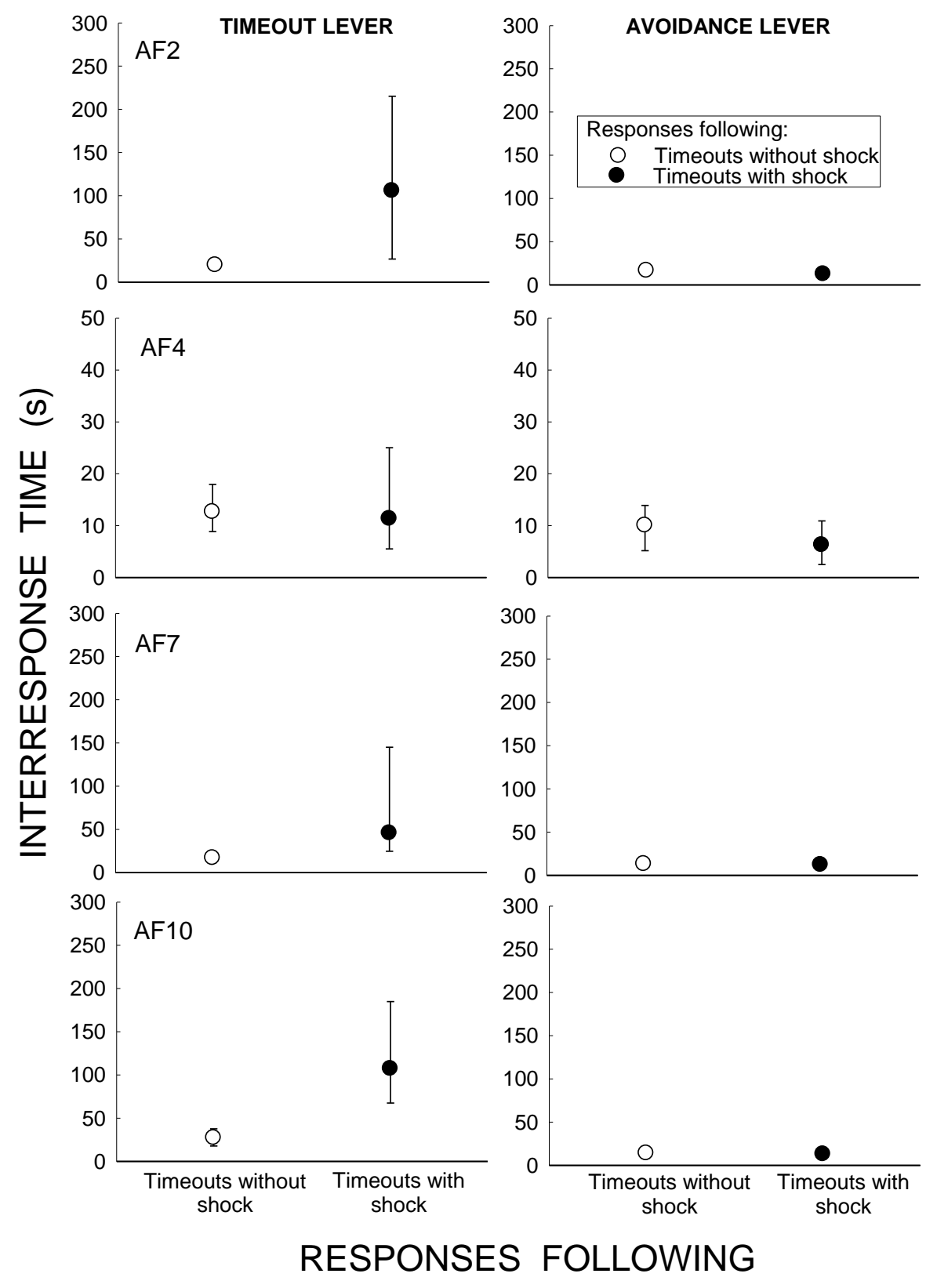

Figure 12. Experiment 2. Median interresponse times (IRTs) on the avoidance (right column) and timeout (left column) levers in each condition for responses following timeouts with shock and timeouts without shock. The IRTs from each of the last 10 sessions of each condition were pooled. Error bars represent the interquartile ranges. 


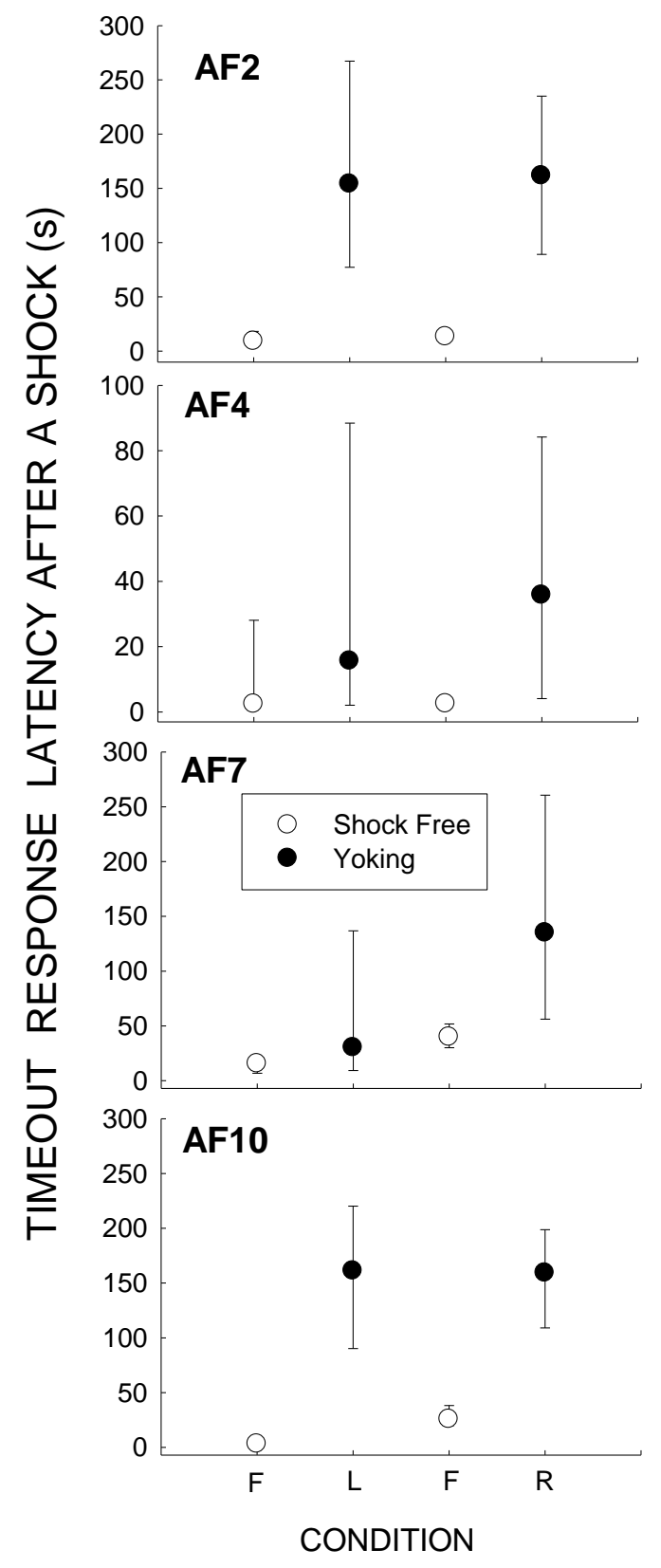

Figure 13. Experiment 2. Median post-shock response latency in the Shock-free (F) baseline (open circles) and in the two yoking conditions: Local Yoking (L) and Random Local Yoking (R) (filled circles) conditions. Data are from the last 10 sessions of each condition, and each data point represents the aggregated replications of the conditions. Error bars represent the interquartile ranges. 


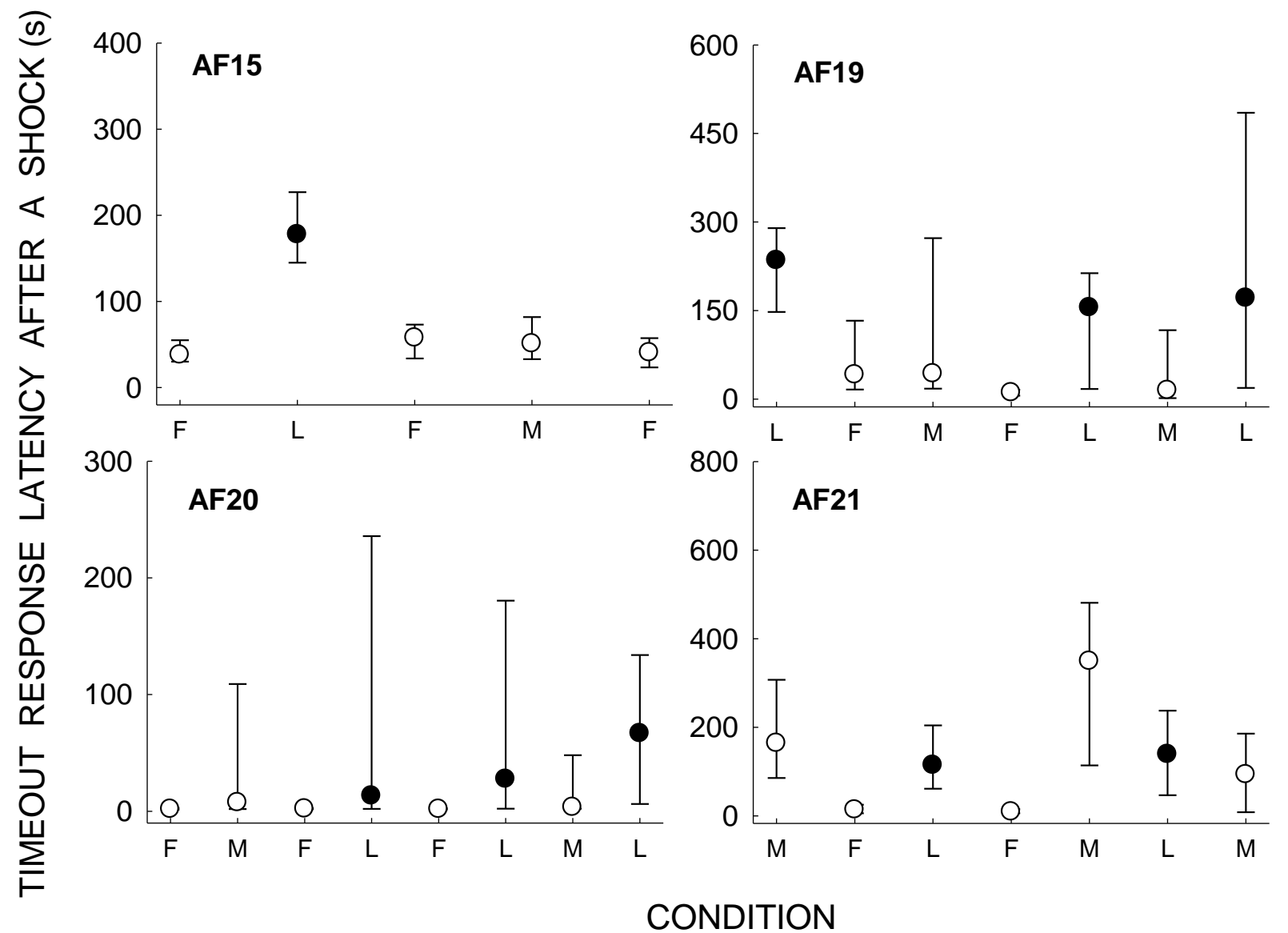

Figure 14. Data from Foreman (2009). Median post-shock response latency in the Shock-free (F) baseline and Molar-Yoking (M) (closed circles), and Local-Yoking (L) (open circles) conditions. Data are from the stable sessions of each condition. Error bars represent the interquartile ranges. 


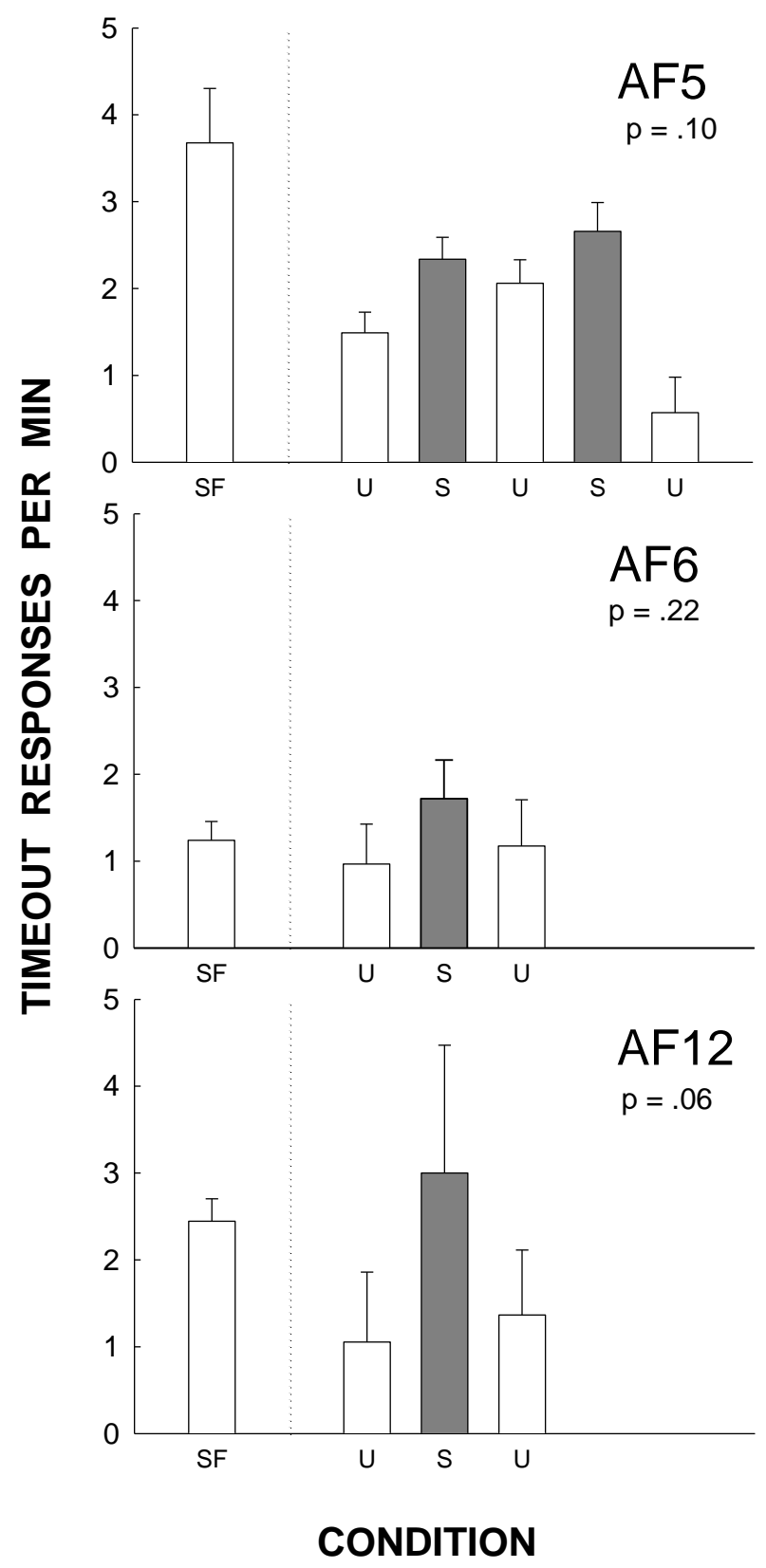

Figure 15. Experiment 3. Mean timeout responses per min for the last 10 sessions of the unsignaled (U) and signaled (S) conditions. The mean timeout response rates in the Shock-Free (SF) baseline of Experiment 1 are also included. Error bars represent one standard deviation. 

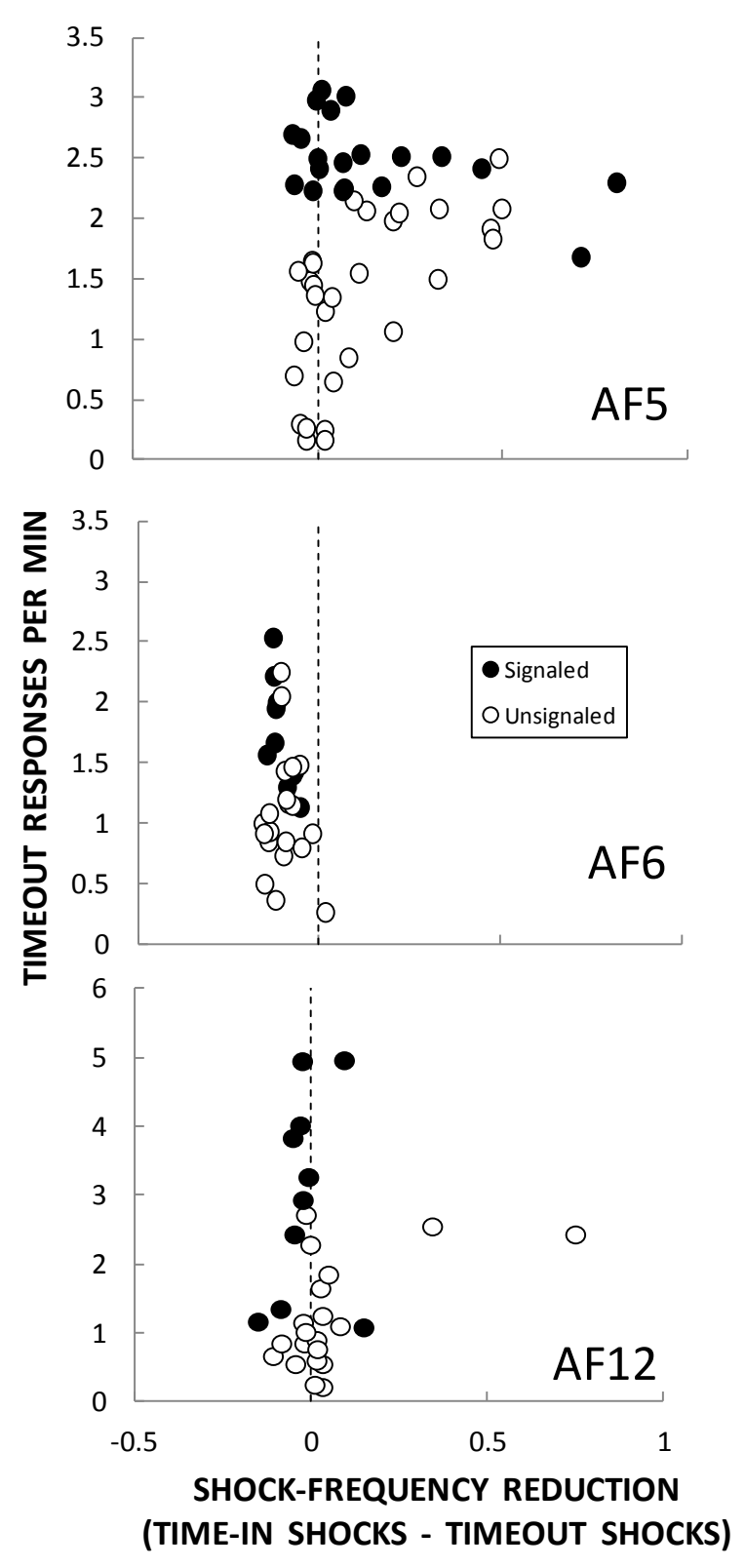

Figure 16. Experiment 3. Timeout responding plotted against shock-frequency reduction. Each data point represents performance in one of the last 10 sessions of each condition. Shockfrequency reduction values were calculated by subtracting the mean timeout shock rate for a session from the mean time-in shock rate for that session. Note the different X-axis for Rat AF12. 

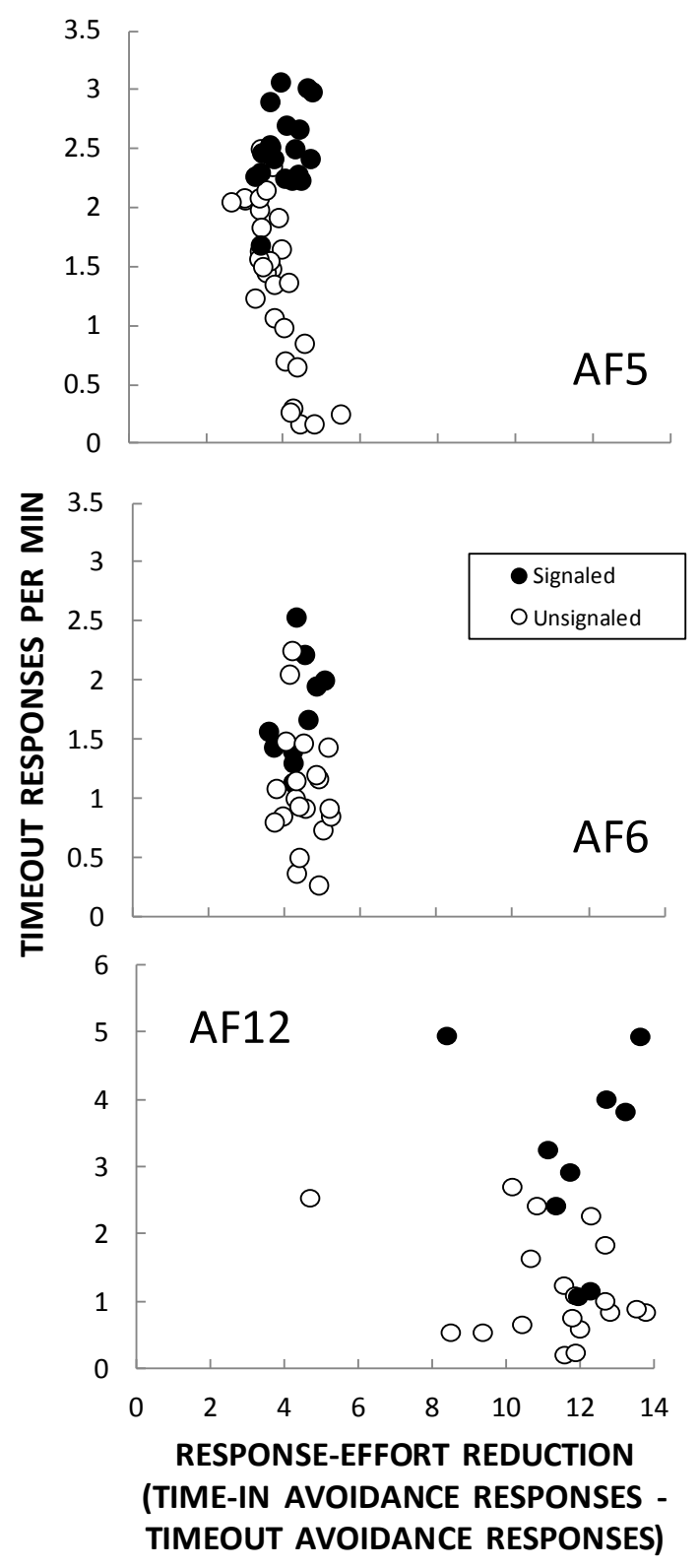

Figure 17. Experiment 3. Timeout responding plotted against response-effort reduction. Each data point represents performance in one of the last 10 sessions of each condition. Response effort reduction values were calculated by subtracting the mean timeout avoidance response rate for a session from the mean time-in avoidance response rate for that session. 


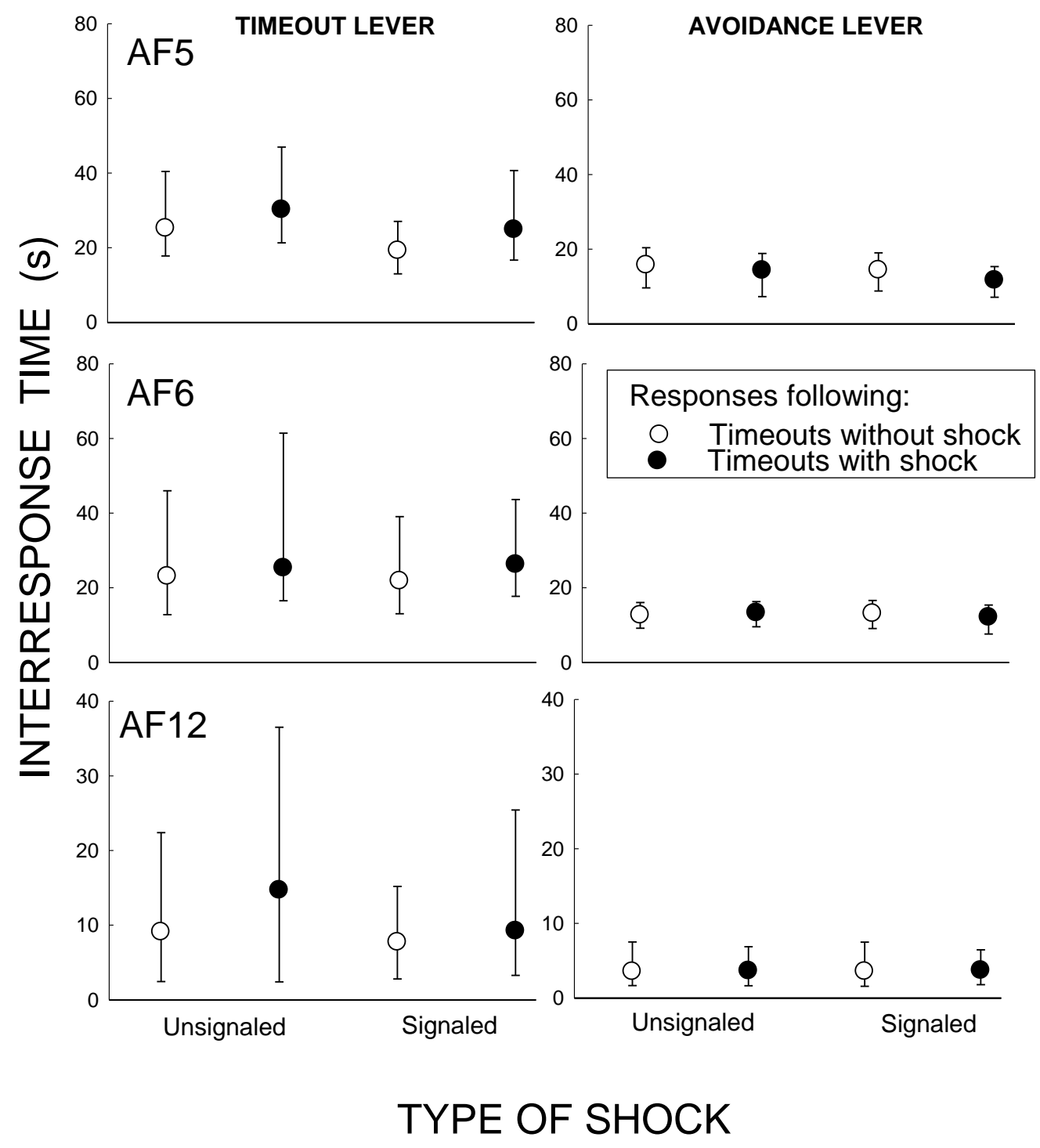

Figure 18. Experiment 3. Median interresponse times (IRTs) on the timeout (left column) and avoidance (right column) levers in each condition for responses following timeouts with shock and timeouts without shock. The IRTs from each of the last 10 sessions of each condition were pooled. Error bars represent the interquartile ranges. 